\title{
IMPROVED LABORATORY TRANSITION PROBABILITIES FOR Ce II, APPLICATION TO THE CERIUM ABUNDANCES OF THE SUN AND FIVE $r$-PROCESS-RICH, METAL-POOR STARS, AND RARE EARTH LAB DATA SUMMARY
}

\author{
J. E. Lawler ${ }^{1}$, C. Sneden ${ }^{2,3}$, J. J. Cowan ${ }^{4}$, I. I. Ivans ${ }^{5,6}$, And E. A. Den Hartog ${ }^{1}$ \\ ${ }^{1}$ Department of Physics, University of Wisconsin, Madison, WI 53706, USA; jelawler@wisc.edu, eadenhar@wisc.edu \\ ${ }^{2}$ Department of Astronomy and McDonald Observatory, University of Texas, Austin, TX 78712, USA \\ ${ }^{3}$ INAF, Osservatorio Astronomico di Padova, Vicolo Osservatorio 5, I-35122 Padova, Italy; chris@ verdi.as.utexas.edu \\ ${ }^{4}$ Homer L. Dodge Department of Physics and Astronomy, University of Oklahoma, Norman, OK 73019, USA; cowan@nhn.ou.edu \\ ${ }^{5}$ The Observatories of the Carnegie Institution of Washington, 813 Santa Barbara St., Pasadena, CA 91101, USA \\ ${ }^{6}$ Princeton University Observatory, Peyton Hall, Princeton, NJ 08544, USA; iii@ ociw.edu \\ Received 2008 November 20; accepted 2009 March 16; published 2009 April 15
}

\begin{abstract}
Recent radiative lifetime measurements accurate to $\pm 5 \%$ using laser-induced fluorescence (LIF) on 43 evenparity and 15 odd-parity levels of $\mathrm{Ce}$ II have been combined with new branching fractions measured using a Fourier transform spectrometer (FTS) to determine transition probabilities for 921 lines of Ce II. This improved laboratory data set has been used to determine a new solar photospheric Ce abundance, $\log \varepsilon=1.61 \pm 0.01$ ( $\sigma=0.06$ from 45 lines), a value in excellent agreement with the recommended meteoritic abundance, log $\varepsilon=1.61 \pm 0.02$. Revised Ce abundances have also been derived for the $r$-process-rich metal-poor giant stars BD $+17^{\circ} 3248$, CS 22892-052, CS 31082-001, HD 115444, and HD 221170. Between 26 and 40 lines were used for determining the $\mathrm{Ce}$ abundance in these five stars, yielding a small statistical uncertainty of \pm 0.01 dex similar to the solar result. The relative abundances in the metal-poor stars of $\mathrm{Ce}$ and $\mathrm{Eu}$, a nearly pure $r$-process element in the Sun, matches $r$-process-only model predictions for solar system material. This consistent match with small scatter over a wide range of stellar metallicities lends support to these predictions of elemental fractions. A companion paper includes an interpretation of these new precision abundance results for $\mathrm{Ce}$ as well as new abundance results and interpretation for Pr, Dy, and Tm.

Key words: atomic data - Galaxy: evolution - nuclear reactions, nucleosynthesis, abundances - stars: abundances - stars: individual (BD+17³248, CS 22892-052, CS 31082-001, HD 115444, HD 221170) - stars: Population II - Sun: abundances
\end{abstract}

Online-only material: color figure, machine-readable tables

\section{INTRODUCTION}

The study of elemental abundances in stellar photospheres continues to be a rich area of investigation. Halo stars are among the oldest objects in the Galaxy and these stars provide a "fossil" record of the Galactic chemical evolution. The discovery and detailed study of a class of metal-poor Galactic halo stars with variable $n$ (eutron)-capture elemental abundances (e.g., Sneden et al. 1995; Smith et al. 1995; Cowan et al. 1996; Woolf et al. 1995; Sneden et al. 1996; Burris et al. 2000; Hill et al. 2002, Sneden et al. 2003b) is particularly significant.

Rare-Earth (RE) elements are among the most spectroscopically accessible of the $n$-capture elements. The open f-shell of the RE neutral atoms and ions yields many strong lines in the visible and near-IR where spectral line blending is less of a problem than in the UV, and where ground-based observations are possible. Observations with a high signal-to-noise ratio $(\mathrm{S} / \mathrm{N})$ and a high spectral resolving power on an increasing number of stars are now available from large ground-based telescopes. The quality of these new astronomical data can be fully exploited only if similar quality basic spectroscopic data, especially data on transition probabilities, are available. The combination of improved astronomical data and improved laboratory data has reduced line-to-line and star-to-star scatter in abundance values for many RE elements.

Cerium is the last of the RE elements in need of additional work and it has the richest line spectra of the RE elements. Experimental work since the mid-1990s has emphasized laser- induced fluorescence (LIF) lifetime measurements (e.g., Langhans et al. 1995; Li et al. 2000; Zhang et al. 2001; Xu et al. 2003; Den Hartog \& Lawler 2008). These LIF lifetimes have typically been combined with theoretical branching fractions to determine transition probabilities for individual $\mathrm{Ce}$ II lines (e.g., Zhang et al. 2001; Palmeri et al. 2000; Biémont \& Quinet 2005).

In spite of the somewhat daunting line density, a large set of Ce II branching fractions measurements based on Fourier transform spectrometer (FTS) data is now complete. The branching fraction measurements are combined with the most recent and extensive LIF radiative lifetimes from Den Hartog \& Lawler (2008) to determine transition probabilities for 921 lines of Ce II reported herein.

Although progress has been made in the theoretical determination of atomic transition probabilities, a recent comparison strongly suggests that modern experimental methods yield more reliable atomic transition probabilities (Lawler et al. 2008a). The method used in this study on $\mathrm{Ce}$ is to combine radiative lifetimes from time-resolved LIF (TR-LIF) with emission branching fraction measurements from high resolution data recorded using an FTS. This method for measuring transition probabilities is accurate, flexible, and efficient. The systematic determination of experimental transition probabilities by combining radiative lifetimes from TR-LIF with branching fractions from emission data recorded with an FTS has played a central role in providing the basic atomic data needed for RE abundance determinations. This method yields absolute transition probabilities which are accurate to $\pm 5 \%(\sim 0.02 \mathrm{dex})$ for strong lines. 
These laboratory data are applied to re-determine the solar abundance of $\mathrm{Ce}$ and to refine the $\mathrm{Ce}$ abundance in five $r$ process-rich, metal-poor Galactic halo stars. The Appendix to this paper includes a summary to all of our RE lab data in machine-readable (MR) form. This work on Ce II completes a multi-year effort to improve laboratory spectroscopic data for RE ions and to apply these data in abundance studies (Lawler et al. 2008b and references therein).

The emergence of a tightly defined $r$-process-only abundance pattern in many very metal-poor Galactic halo stars, at least for the RE elements, has been an exciting development (e.g., Sneden et al. 2003a; Ivans et al. 2006; Lawler et al. 2006; Den Hartog et al. 2006). As this abundance pattern becomes even more tightly defined, it will: (1) provide a powerful constraint on future modeling of the $r$-process nucleosynthesis, (2) help determine a definitive $r$-process site, and (3) unlock other details of the $r$-process and of the Galactic chemical evolution.

\section{CE II BRANCHING FRACTIONS AND ATOMIC TRANSITION PROBABILITIES}

As discussed above, radiative lifetimes from Den Hartog \& Lawler (2008) provide a foundation for this study of branching fractions and the transition probabilities of Ce II. Branching fraction measurements were attempted on lines from all 74 levels of the lifetime experiment, and were completed for lines from 43 even-parity and 15 odd-parity upper levels. As in earlier work on other RE spectra, we used the $1.0 \mathrm{~m}$ FTS at the National Solar Observatory (NSO) on Kitt Peak for Ce II branching fraction measurements. The Kitt Peak FTS has the large etendue of all interferometric spectrometers, a limit of resolution as small as $0.01 \mathrm{~cm}^{-1}$, wavenumber accuracy to 1 part in $10^{8}$, broad spectral coverage from the UV to IR, and the capability of recording a million point spectrum in 10 minutes (Brault 1976). The line density in Ce II is so high that a very high performance FTS is essential. Grating spectrographs are not adequate.

\subsection{Energy Levels of Ce II}

Figure 1 shows a partial Grotrian diagram constructed from the energy level compilation of Martin et al. (1978) for singly ionized Ce. A total of 288 even-parity and 192 odd-parity levels are included in the compilation. Most of the low-lying $\left(<20,000 \mathrm{~cm}^{-1}\right)$ energy levels are known and have assignments. Although Ce II is the most complex of the singly ionized RE spectra, classical analyses of the spectrum have yielded extensive results (e.g., Martin et al. and reference therein). The absence of hyperfine structure (hfs) results in narrow spectral lines and a tolerable amount of line blending for such a rich spectrum.

There are three low odd-parity configurations including the $4 \mathrm{f}\left({ }^{2} \mathrm{~F}\right) 5 \mathrm{~d}^{2}, 4 \mathrm{f}\left({ }^{2} \mathrm{~F}\right) 6 \mathrm{~s}^{2}$, and $4 \mathrm{f}\left({ }^{2} \mathrm{~F}\right) 5 \mathrm{~d} 6 \mathrm{~s}$ which contribute over 100 levels below $20,000 \mathrm{~cm}^{-1}$ including the ground level. This near degeneracy of the $4 \mathrm{f}, 5 \mathrm{~d}$, and 6 s orbitals is typical of REs and it leads to substantial configuration mixing. Of the 118 lowlying odd-parity levels listed in Martin et al. (1978), 74 are assigned to the $4 \mathrm{f}\left({ }^{2} \mathrm{~F}\right) 5 \mathrm{~d}^{2}$ configuration, two are assigned to the $4 f\left({ }^{2} \mathrm{~F}\right) 6 \mathrm{~s}^{2}$ configuration, and 42 are assigned to the $4 \mathrm{f}\left({ }^{2} \mathrm{~F}\right) 5 \mathrm{~d} 6 \mathrm{~s}$ configuration. Not all of these 118 levels have term assignments, but a counting of angular momentum projections indicates that very few (perhaps only 2) levels from these three configurations are unobserved. There is a gap of about $5700 \mathrm{~cm}^{-1}$ before the $4 \mathrm{f}^{2} 6 \mathrm{p}$ configuration starts at $25,766 \mathrm{~cm}^{-1}$. The nearly complete knowledge of low odd-parity levels simplifies the branching

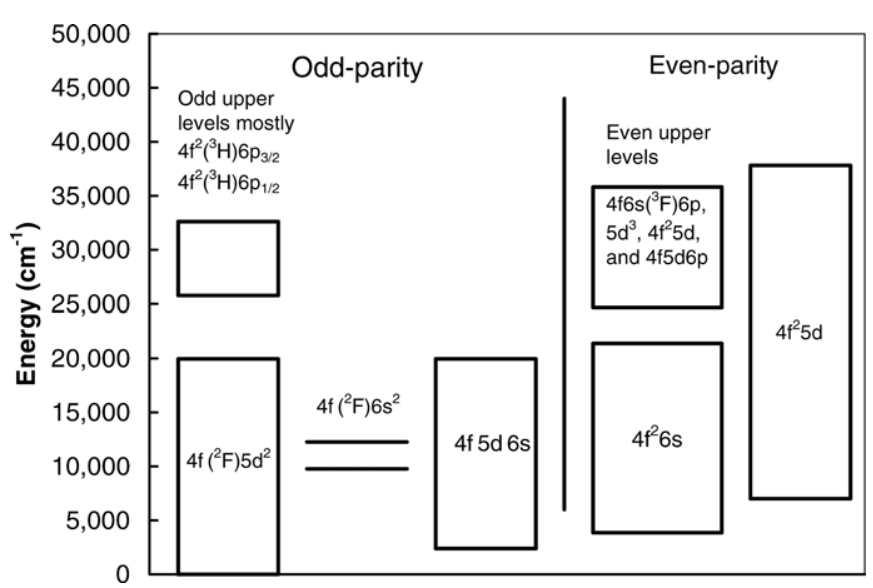

Figure 1. Partial Grotrian diagram for Ce II. Upper and lower levels of both parities included in this study are shown.

fraction study, since there is little chance of missing a strong branch to an unobserved lower level. Even-parity upper levels of this study are between 24,000 and $33,000 \mathrm{~cm}^{-1}$ and all have short, $<9$ ns, radiative lifetimes. Due to the frequency cubed scaling of transition probabilities, there is no real possibility of near-IR branches to higher unobserved levels contributing more than a small fraction of $1 \%$ to the total decay of such short lived levels.

Two configurations, the $4 \mathrm{f}^{2} 6 \mathrm{~s}$ and $4 \mathrm{f}^{2} 5 \mathrm{~d}$, contribute low-lying even-parity levels. Although the $4 \mathrm{f}^{2} 6 \mathrm{~s}$ configuration extends from $3800 \mathrm{~cm}^{-1}$ to just over $21,000 \mathrm{~cm}^{-1}$, the $4 \mathrm{f}^{2} 5 \mathrm{~d}$ extends up to nearly $40,000 \mathrm{~cm}^{-1}$. There are 99 known even-parity levels below $25,000 \mathrm{~cm}^{-1}$. A counting of angular momentum projections including levels of the $4 \mathrm{f}^{2} 5 \mathrm{~d}$ configuration up to $40,000 \mathrm{~cm}^{-1}$ indicates that few (perhaps only 4) levels of the two low even-parity configurations are unobserved. These unobserved even-parity levels are low J levels $(3 / 2$ or $1 / 2)$ and are likely around $20,000 \mathrm{~cm}^{-1}$ or higher. Two of the missing $J=1 / 2$ levels were found by Palmeri et al. (2000). This situation is similar to the other parity; there is a nearly complete knowledge of low even-parity levels. Odd-parity upper levels of this study are between 25,000 and $33,000 \mathrm{~cm}^{-1}$ and all have short, $<9 \mathrm{~ns}$, radiative lifetimes. Due to the frequency cubed scaling of transition probabilities, there is again no possibility of near-IR branches to higher unobserved levels contributing more than a small fraction of $1 \%$ to the total decay of such short lived levels. This nearly complete knowledge, including assignments, of the low-lying $\left(<20,000 \mathrm{~cm}^{-1}\right)$ energy levels of both parities in Ce II greatly simplifies the branching fraction analysis.

\subsection{Ce II Branching Fraction Analysis and Relative Radiometric Calibration}

As in earlier studies our experimental branching fractions are based on a large set of FTS data including: spectra of lamps at high currents to reveal very weak branches to known levels, good IR spectra to reveal weak IR branches to known levels, and low current spectra in which dominant branches are optically thin covering the UV to near-IR. Table 1 is a list of the 14 FTS spectra used in our branching fraction study. All were recorded using the National Solar Observatory 1.0 m FTS on Kitt Peak. Most of these spectra, \#1-9, were recorded during a February 2002 observing run using Hollow Cathode Discharge (HCD) lamps. Visible spectra of an Electrodeless Discharge Lamp (EDL), \#10-11, have quite good S/N on weaker lines. Lines 
Table 1

Fourier Transform Spectra of Ce Lamps used in This Study. All Were Recorded Using the $1.0 \mathrm{~m}$ FTS on the McMath Telescope at the National Solar Observatory, Kitt Peak, AZ

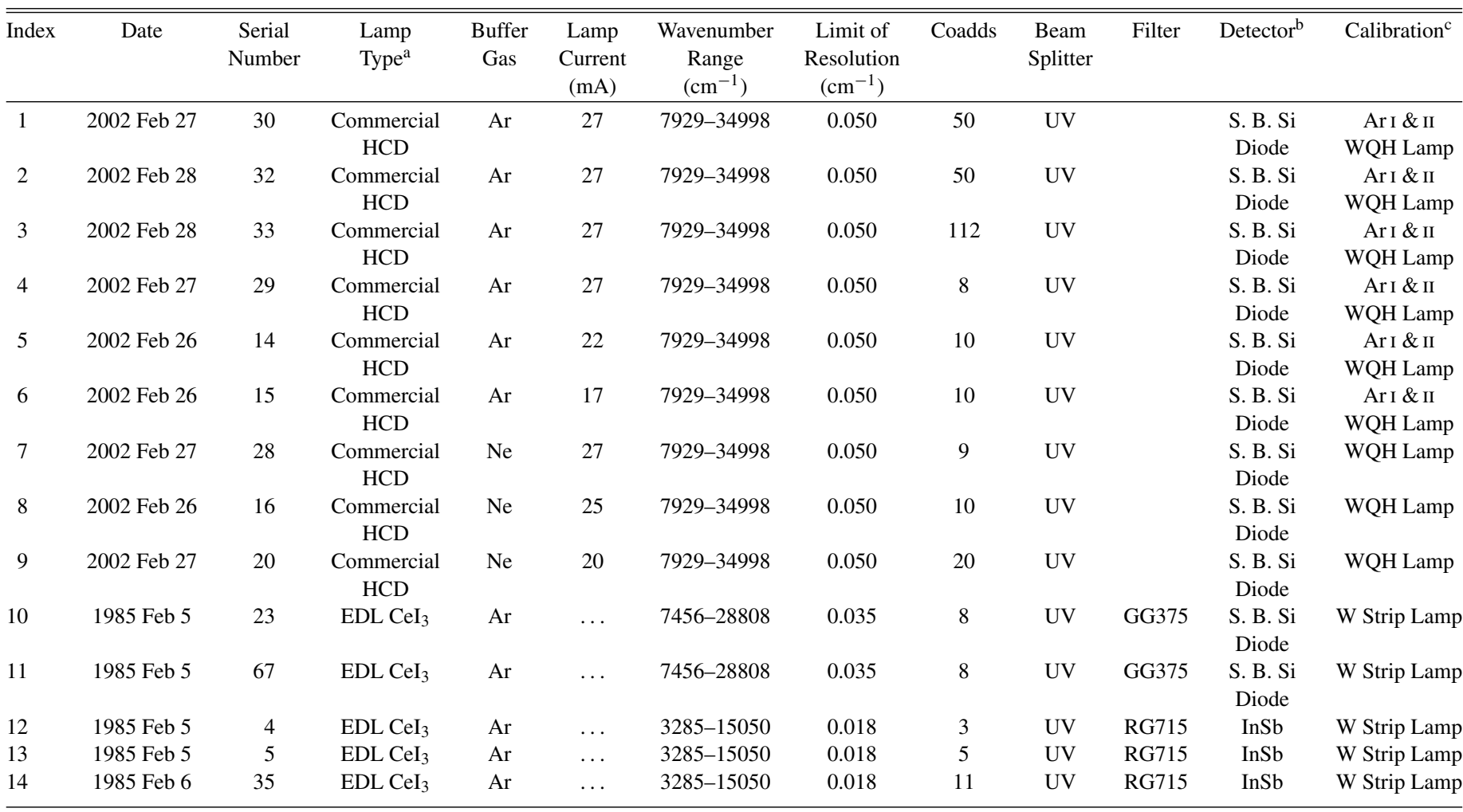

Notes.

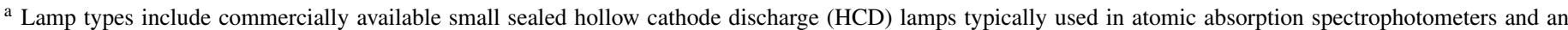
electrodeless discharge lamp (EDL) run with isotopically pure ${ }^{140} \mathrm{Ce}$ introduced as an iodide salt.

b Detectors types include the super blue (S. B.) Si photodiode and Indium antimonide (InSb) detector for the near infrared.

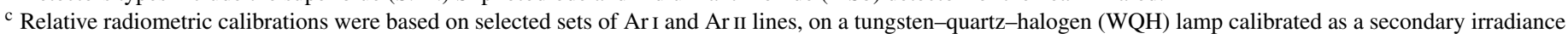
standard, and on a tungsten (W) Strip Lamp calibrated as a secondary radiance standard.

of $\mathrm{Ce}$ II in comparison to lines of $\mathrm{Ce} \mathrm{I}$ are stronger in spectra \#10-11 than in spectra \#1-9 and this effect was very valuable in assessing possible blends of Ce II and Ce I lines. Spectra of Ce/ Ne HCD lamps were used only to assess and, when necessary, correct for blends between $\mathrm{Ce}$ II and Ar lines. Since no Ar II or Ar I lines appeared in the EDL spectra, these could also be used to assess and correct for blends between Ce II and Ar lines. This redundancy is important since many cases of possible blends involving Ce II, Ce I, and Ar lines occur. No significant optical depth effects on Ce II lines appear in any of the spectra, probably due to the rich level structure and large partition function of this ion. Spectra \#12-14 yielded measurements on a few near-IR lines, but generally the near IR lines from the short lived levels of this study have negligible branching fractions. All 14 raw spectra are available from the electronic archives of the National Solar Observatory. ${ }^{7}$

The establishment of an accurate relative radiometric calibration or efficiency is critical to a branching fraction experiment. As indicated in Table 1, we made use of both standard lamp calibrations and Ar I and Ar II line calibrations in this Ce II study. Tungsten (W) filament standard lamps are particularly useful near the Si detector cutoff in the 10,000 to $9000 \mathrm{~cm}^{-1}$ range where the FTS sensitivity is changing rapidly as a function of wavenumber, and near the dip in sensitivity at $12,500 \mathrm{~cm}^{-1}$ from the aluminum coated optics. Tungsten lamps

\footnotetext{
7 Available at http://nsokp.nso.edu/.
}

are not bright enough to be useful for FTS calibrations in the UV region, and blue or UV branches typically dominate the decay of levels studied using our lifetime experiment. In general one must be careful when using continuum lamps to calibrate the FTS over wide spectral ranges, because the "ghost" of a continuum is a continuum. The ArI and Ar II line technique, which is internal to the HCD Ce/Ar lamp spectra, is still our preferred calibration technique. It captures the wavelength-dependent response of detectors, spectrometer optics, lamp windows, and any other components in the light path or any reflections which contribute to the detected signal (such as due to light reflecting off the back of the hollow cathode). This calibration technique is based on a comparison of well-known branching ratios for sets of Ar I and Ar II lines widely separated in wavelength, to the intensities measured for the same lines. Sets of Ar I and Ar II lines have been established for this purpose in the range of 4300-35000 $\mathrm{cm}^{-1}$ by Adams \& Whaling (1981); Danzmann \& Kock (1982); Hashiguchi \& Hasikuni (1985), and Whaling et al. (1993). The $\mathrm{Ce} / \mathrm{Ne}$ spectra from 2002 and the EDL spectra from 1985 could only be calibrated using W standard lamps. The older W lamp is a strip lamp calibrated as a spectral radiance (Watts $\left(\mathrm{m}^{2} \mathrm{sr}\right.$ $\mathrm{nm})^{-1}$ ) standard, and the newer is a tungsten-quartz-halogen lamp calibrated as a spectral irradiance (Watts $\left(\mathrm{m}^{2} \mathrm{~nm}\right)^{-1}$ at a specified distance) standard. Neither of these W filament lamps is hot or bright enough to yield a reliable UV calibration, but they are useful in the visible and near IR for interpolation and as a redundant calibration. 
All possible transition wavenumbers between known energy levels of Ce II satisfying both the parity change and $\Delta J=$ $-1,0$, or 1 selection rules were computed and used during analysis of FTS data. Energy levels from Martin et al. (1978) were used to determine possible transition wavenumbers. Levels from Martin et al. (1978) are available in electronic form from Martin et al. (2000). ${ }^{8}$ Systematic errors from missing branches to known lower levels are negligible in our work, because the level structure of $\mathrm{Ce}$ II is so well known and because we were able to make measurements on branching fractions of 0.01 or smaller. All naturally occurring $\mathrm{Ce}$ isotopes are even (nuclear spin $I=0$ ) isotopes: ${ }^{136} \mathrm{Ce}$ (abundance $\left.0.185 \%\right),{ }^{138} \mathrm{Ce}$ (abundance $0.251 \%$ ), ${ }^{140} \mathrm{Ce}$ (abundance $88.450 \%$ ), and ${ }^{142} \mathrm{Ce}$ (abundance $11.114 \%$ ) (Böhlke et al. 2005). Lines of Ce II are generally quite narrow because they have no hfs and small isotope shifts.

Branching fraction measurements were completed for lines from 43 even-parity and 15 odd-parity upper levels of the 74 levels studied in the lifetime experiment by Den Hartog \& Lawler (2008). The levels for which branching fractions could not be completed had a strong branch beyond the UV limit of our spectra, or had a strong branch which was severely blended. Typically an even-parity upper level, depending on its $J$ value, has about 55 possible transitions to known lower levels, and an odd-parity upper level has about 40 possible transitions to known lower levels. More than 30,000 possible spectral line observations were studied during the analysis of 14 different $\mathrm{Ce} / \mathrm{Ar}$ and $\mathrm{Ce} / \mathrm{Ne}$ spectra. We set integration limits and occasionally nonzero baselines "interactively" during analysis of the FTS spectra. An occasional nonzero baseline is needed when a weak line is located on a line wing of a much stronger line. Weak red and near-IR lines of Ce II in the EDL spectra also require a nonzero baseline due to some continuum emission from this lamp. The same numerical integration routine was used to determine the un-calibrated intensities of Ce II lines and selected ArI and ArII lines used to establish a relative radiometric calibration of the spectra. A simple numerical integration technique was used in this and most of our other RE studies because of weakly resolved or unresolved hyperfine and isotopic structure. More sophisticated profile fitting is used only when the line subcomponent structure is either fully resolved in the FTS data or known from independent measurements.

\subsection{Branching Fraction Uncertainties}

The procedure for determining branching fraction uncertainties was described in detail by Wickliffe et al. (2000). Branching fractions from a given upper level are defined to sum to unity, thus a dominant line from an upper level has small branching fraction uncertainty almost by definition. Branching fractions for weaker lines near the dominant line(s) tend to have uncertainties limited by their S/N. Systematic uncertainties in the radiometric calibration are typically the dominant source of uncertainty for widely separated lines from a common upper level. We used a formula for estimating this systematic uncertainty that was presented and tested extensively by Wickliffe et al. (2000). The EDL spectra enabled us to include measurements of quite weak branches in the visible and near-IR. Uncertainties on branching fractions of the weak visible and near-IR lines are larger than uncertainties on the dominant branches. In the final analysis, the branching fraction uncertainties are primarily systematic. Redundant measurements with independent radiometric calibrations help in the assessment of systematic

\footnotetext{
8 Available at http://physics.nist.gov/PhysRefData/ASD/index.html.
}

uncertainties. Redundant measurements from spectra with different discharge conditions also make it easier to spot blended lines. Typically some fraction of a weak blended feature with a $1 \%$ or less branching fraction was included in the branching fraction normalization, but the blended line was omitted from the final table of transition probabilities.

\subsection{Ce II Atomic Transition Probabilities}

Branching fractions from the FTS spectra were combined with the radiative lifetime measurements (Den Hartog \& Lawler 2008) to determine absolute transition probabilities for 921 lines of Ce II in Table 2. Air wavelengths in Table 2 were computed from energy levels (Martin et al. 1978) using the standard index of air (Edlén 1953). Parities are included in Table 2 using "ev" and "od" notation and decimal notation for $J$ values which are compatible with our MR table of transition probabilities.

Transition probabilities for the very weakest lines (branching fractions $\sim 0.001$ or weaker) which were observed with poor $\mathrm{S} / \mathrm{N}$ and for weak blended lines (branching fractions $\leqslant 0.01$ ) are not included in Table 2; however, these lines are included in the branching fraction normalization. The effect of the problem lines becomes apparent if one sums all transition probabilities in Table 2 from a chosen upper level, and compares the sum to the inverse of the upper level lifetime from Den Hartog \& Lawler (2008). Typically the sum of the Table 2 transition probabilities is between $90 \%$ and $100 \%$ of the inverse lifetime. Although there is significant fractional uncertainty in the branching fractions for these problem lines, this does not have much effect on the uncertainty of the stronger lines that were kept in Table 2. Branching fraction uncertainties are combined in quadrature with lifetime uncertainties to determine the transition probability uncertainties in Table 2 .

\subsection{Comparisons to Other Data Sets}

Three large-scale theoretical or semiempirical sets of Ce II transition probabilities are available for comparison to our new experimental results. Figure 2 shows comparisons of our new experimental transition probabilities to theoretical values from Fawcett (1990). A total of 686 lines in common are included in the Fawcett comparisons. Fawcett's results are based on ab initio calculations of Slater parameters which were leastsquare adjusted to experimental energies. He also included configuration mixing. Figure 3 shows comparisons of our results to theoretical transition probabilities recently downloaded from the DREAM database (Biémont \& Quinet 2005).${ }^{9}$ Earlier work by some of the same team members (Palmeri et al. 2000; Zhang et al. 2001) is included in the more extensive online data set. A total of 890 lines in common are included in the Biémont comparisons. The DREAM results may not be purely theoretical due to the use of experimental LIF lifetimes to rescale theoretical transition probabilities in some cases (e.g., Zhang et al. 2001). Figure 4 shows comparisons of our results to recently downloaded semiempirical results from Kurucz (1998). ${ }^{10}$ A total of 529 lines in common are included in the Kurucz comparisons. It is interesting that the comparisons to semiempirical results from Kurucz show the best agreement. It is not possible to draw conclusions about a particular theoretical method because the Kurucz database includes data from a number of sources.

\footnotetext{
9 Available at http://w3.umh.ac.be/ astro/dream.shtml.

10 Available at http://kurucz.harvard.edu/.
} 
Table 2

Atomic Transition Probabilities for Ce II Organized by Increasing Wavelength in Air, $\lambda_{\text {air }}$

\begin{tabular}{|c|c|c|c|c|c|c|c|c|}
\hline$\lambda_{\text {air }}(\AA)$ & $E_{\text {upper }}\left(\mathrm{cm}^{-1}\right)$ & Parity & $J_{\text {upp }}$ & $E_{\text {lower }}\left(\mathrm{cm}^{-1}\right)$ & Parity & $J_{\text {low }}$ & $A$-value $\left(10^{6} \mathrm{~s}^{-1}\right)$ & $\log (g f)$ \\
\hline 3422.705 & 32802.165 & ev & 5.5 & 3593.882 & od & 4.5 & $19.4 \pm 2.4$ & -0.39 \\
\hline 3426.205 & 30166.057 & ev & 3.5 & 987.611 & od & 4.5 & $15.5 \pm 1.5$ & -0.66 \\
\hline 3485.053 & 28685.758 & ev & 2.5 & 0.000 & od & 3.5 & $24.9 \pm 1.5$ & -0.57 \\
\hline 3507.941 & 29908.904 & ev & 4.5 & 1410.304 & od & 4.5 & $6.0 \pm 1.0$ & -0.96 \\
\hline 3520.520 & 29807.078 & ev & 3.5 & 1410.304 & od & 4.5 & $8.3 \pm 0.8$ & -0.91 \\
\hline 3530.019 & 30702.610 & ev & 4.5 & 2382.246 & od & 4.5 & $8.6 \pm 0.9$ & -0.80 \\
\hline 3534.045 & 32492.038 & ev & 5.5 & 4203.934 & od & 6.5 & $32.4 \pm 2.7$ & -0.14 \\
\hline 3539.079 & 30829.124 & ev & 3.5 & 2581.257 & od & 4.5 & $35.7 \pm 2.7$ & -0.27 \\
\hline 3552.724 & 30702.610 & ev & 4.5 & 2563.233 & od & 5.5 & $9.8 \pm 0.9$ & -0.73 \\
\hline 3555.001 & 30702.610 & ev & 4.5 & 2581.257 & od & 4.5 & $15.5 \pm 1.5$ & -0.53 \\
\hline 3560.802 & 33531.388 & ev & 6.5 & 5455.845 & od & 7.5 & $73 \pm 4$ & 0.29 \\
\hline 3577.456 & 31738.484 & ev & 5.5 & 3793.634 & od & 6.5 & $60 \pm 4$ & 0.14 \\
\hline 3580.565 & 29794.517 & ev & 3.5 & 1873.934 & od & 3.5 & $3.3 \pm 0.4$ & -1.30 \\
\hline 3604.195 & 28725.148 & ev & 4.5 & 987.611 & od & 4.5 & $2.6 \pm 0.4$ & -1.30 \\
\hline 3632.092 & 30166.057 & ev & 3.5 & 2641.559 & od & 3.5 & $7.1 \pm 0.8$ & -0.95 \\
\hline 3646.962 & 29794.517 & ev & 3.5 & 2382.246 & od & 4.5 & $15.0 \pm 1.3$ & -0.62 \\
\hline 3653.104 & 30245.878 & ev & 4.5 & 2879.695 & od & 5.5 & $16.2 \pm 1.8$ & -0.49 \\
\hline 3653.664 & 31155.623 & ev & 6.5 & 3793.634 & od & 6.5 & $22.8 \pm 1.6$ & -0.20 \\
\hline 3654.934 & 29994.041 & ev & 2.5 & 2641.559 & od & 3.5 & $12.5 \pm 1.4$ & -0.82 \\
\hline 3655.844 & 29908.904 & ev & 4.5 & 2563.233 & od & 5.5 & $44.9 \pm 2.6$ & -0.05 \\
\hline 3658.256 & 29908.904 & ev & 4.5 & 2581.257 & od & 4.5 & $1.95 \pm 0.21$ & -1.41 \\
\hline 3659.225 & 28730.712 & ev & 3.5 & 1410.304 & od & 4.5 & $13.2 \pm 1.0$ & -0.67 \\
\hline 3659.970 & 28725.148 & ev & 4.5 & 1410.304 & od & 4.5 & $9.2 \pm 1.0$ & -0.73 \\
\hline 3660.638 & 28297.473 & ev & 3.5 & 987.611 & od & 4.5 & $19.8 \pm 1.4$ & -0.50 \\
\hline 3661.907 & 30180.096 & ev & 6.5 & 2879.695 & od & 5.5 & $1.32 \pm 0.12$ & -1.43 \\
\hline 3665.488 & 30637.157 & ev & 2.5 & 3363.427 & od & 2.5 & $1.10 \pm 0.18$ & -1.88 \\
\hline 3666.346 & 29908.904 & ev & 4.5 & 2641.559 & od & 3.5 & $0.97 \pm 0.11$ & -1.71 \\
\hline 3667.978 & 30134.910 & ev & 5.5 & 2879.695 & od & 5.5 & $28.2 \pm 1.9$ & -0.17 \\
\hline 3668.724 & 27249.669 & ev & 2.5 & 0.000 & od & 3.5 & $4.8 \pm 0.4$ & -1.23 \\
\hline 3670.668 & 30829.124 & ev & 3.5 & 3593.882 & od & 4.5 & $2.15 \pm 0.19$ & -1.46 \\
\hline 3671.938 & 29807.078 & ev & 3.5 & 2581.257 & od & 4.5 & $5.5 \pm 0.6$ & -1.05 \\
\hline 3672.155 & 28634.516 & ev & 5.5 & 1410.304 & od & 4.5 & $3.0 \pm 0.5$ & -1.14 \\
\hline 3673.633 & 29794.517 & ev & 3.5 & 2581.257 & od & 4.5 & $6.2 \pm 0.6$ & -1.00 \\
\hline 3679.156 & 29807.078 & ev & 3.5 & 2634.666 & od & 2.5 & $4.24 \pm 0.28$ & -1.16 \\
\hline 3680.089 & 29807.078 & ev & 3.5 & 2641.559 & od & 3.5 & $6.4 \pm 0.5$ & -0.98 \\
\hline 3680.857 & 29794.517 & ev & 3.5 & 2634.666 & od & 2.5 & $2.17 \pm 0.14$ & -1.45 \\
\hline 3682.083 & 32802.165 & ev & 5.5 & 5651.357 & od & 5.5 & $6.2 \pm 0.7$ & -0.82 \\
\hline 3685.515 & 30829.124 & ev & 3.5 & 3703.594 & od & 3.5 & $1.48 \pm 0.17$ & -1.62 \\
\hline 3687.799 & 30702.610 & ev & 4.5 & 3593.882 & od & 4.5 & $5.9 \pm 0.4$ & -0.92 \\
\hline 3694.908 & 29438.817 & ev & 5.5 & 2382.246 & od & 4.5 & $5.0 \pm 0.5$ & -0.91 \\
\hline 3702.785 & 30702.610 & ev & 4.5 & 3703.594 & od & 3.5 & $4.2 \pm 0.4$ & -1.06 \\
\hline 3704.976 & 32802.165 & ev & 5.5 & 5819.113 & od & 4.5 & $6.2 \pm 0.8$ & -0.81 \\
\hline 3709.287 & 31155.623 & ev & 6.5 & 4203.934 & od & 6.5 & $29.4 \pm 1.8$ & -0.07 \\
\hline 3709.929 & 27934.638 & ev & 4.5 & 987.611 & od & 4.5 & $26.3 \pm 1.5$ & -0.26 \\
\hline 3716.366 & 26900.354 & ev & 3.5 & 0.000 & od & 3.5 & $31.0 \pm 1.9$ & -0.29 \\
\hline 3719.791 & 29438.817 & ev & 5.5 & 2563.233 & od & 5.5 & $8.0 \pm 0.8$ & -0.70 \\
\hline 3722.100 & 32372.621 & od & 4.5 & 5513.709 & ev & 5.5 & $6.0 \pm 0.6$ & -0.90 \\
\hline 3722.288 & 29438.817 & ev & 5.5 & 2581.257 & od & 4.5 & $5.2 \pm 0.3$ & -0.89 \\
\hline 3722.763 & 29449.778 & ev & 1.5 & 2595.644 & od & 1.5 & $9.3 \pm 0.6$ & -1.11 \\
\hline 3724.629 & 32492.038 & ev & 5.5 & 5651.357 & od & 5.5 & $6.2 \pm 0.5$ & -0.81 \\
\hline 3725.603 & 30829.124 & ev & 3.5 & 3995.460 & od & 3.5 & $1.28 \pm 0.15$ & -1.67 \\
\hline 3725.673 & 32802.165 & ev & 5.5 & 5969.007 & od & 5.5 & $20.0 \pm 2.2$ & -0.30 \\
\hline 3726.456 & 31738.484 & ev & 5.5 & 4910.963 & od & 5.5 & $1.75 \pm 0.18$ & -1.36 \\
\hline 3726.961 & 27811.496 & ev & 3.5 & 987.611 & od & 4.5 & $6.3 \pm 0.5$ & -0.98 \\
\hline 3728.018 & 32492.038 & ev & 5.5 & 5675.763 & od & 4.5 & $29.0 \pm 2.3$ & -0.14 \\
\hline 3728.180 & 29449.778 & ev & 1.5 & 2634.666 & od & 2.5 & $12.1 \pm 1.1$ & -0.99 \\
\hline 3728.417 & 32269.252 & ev & 7.5 & 5455.845 & od & 7.5 & $43.1 \pm 2.9$ & 0.16 \\
\hline 3728.637 & 28685.758 & ev & 2.5 & 1873.934 & od & 3.5 & $3.05 \pm 0.28$ & -1.42 \\
\hline 3729.916 & 30166.057 & ev & 3.5 & 3363.427 & od & 2.5 & $3.1 \pm 0.3$ & -1.29 \\
\hline 3732.462 & 29166.597 & ev & 4.5 & 2382.246 & od & 4.5 & $2.43 \pm 0.21$ & -1.29 \\
\hline 3736.434 & 32372.621 & od & 4.5 & 5616.739 & ev & 4.5 & $2.13 \pm 0.24$ & -1.35 \\
\hline 3744.010 & 30065.164 & ev & 3.5 & 3363.427 & od & 2.5 & $1.75 \pm 0.17$ & -1.53 \\
\hline 3746.256 & 29281.374 & ev & 2.5 & 2595.644 & od & 1.5 & $2.19 \pm 0.17$ & -1.56 \\
\hline 3748.055 & 32492.038 & ev & 5.5 & 5819.113 & od & 4.5 & $17.8 \pm 1.1$ & -0.35 \\
\hline 3750.998 & 30245.878 & ev & 4.5 & 3593.882 & od & 4.5 & $6.5 \pm 0.5$ & -0.86 \\
\hline 3751.742 & 29281.374 & ev & 2.5 & 2634.666 & od & 2.5 & $2.19 \pm 0.16$ & -1.56 \\
\hline
\end{tabular}


Table 2

(Continued)

\begin{tabular}{|c|c|c|c|c|c|c|c|c|}
\hline$\lambda_{\text {air }}(\AA)$ & $E_{\text {upper }}\left(\mathrm{cm}^{-1}\right)$ & Parity & $J_{\text {upp }}$ & $E_{\text {lower }}\left(\mathrm{cm}^{-1}\right)$ & Parity & $J_{\text {low }}$ & $A$-value $\left(10^{6} \mathrm{~s}^{-1}\right)$ & $\log (g f)$ \\
\hline 3752.448 & 30637.157 & ev & 2.5 & 3995.460 & od & 3.5 & $3.5 \pm 0.3$ & -1.35 \\
\hline 3755.789 & 33531.388 & ev & 6.5 & 6913.392 & od & 6.5 & $3.3 \pm 0.5$ & -1.01 \\
\hline 3757.855 & 29166.597 & ev & 4.5 & 2563.233 & od & 5.5 & $6.8 \pm 0.8$ & -0.84 \\
\hline 3760.403 & 29166.597 & ev & 4.5 & 2581.257 & od & 4.5 & $1.39 \pm 0.13$ & -1.53 \\
\hline 3763.604 & 30829.124 & ev & 3.5 & 4266.397 & od & 3.5 & $6.9 \pm 0.5$ & -0.93 \\
\hline 3764.115 & 29438.817 & ev & 5.5 & 2879.695 & od & 5.5 & $21.4 \pm 1.3$ & -0.26 \\
\hline 3766.503 & 30245.878 & ev & 4.5 & 3703.594 & od & 3.5 & $5.5 \pm 0.4$ & -0.94 \\
\hline 3766.681 & 30134.910 & ev & 5.5 & 3593.882 & od & 4.5 & $1.66 \pm 0.17$ & -1.37 \\
\hline 3769.052 & 27934.638 & ev & 4.5 & 1410.304 & od & 4.5 & $1.51 \pm 0.14$ & -1.49 \\
\hline 3771.600 & 30829.124 & ev & 3.5 & 4322.708 & od & 2.5 & $16.7 \pm 1.0$ & -0.55 \\
\hline 3776.606 & 30065.164 & ev & 3.5 & 3593.882 & od & 4.5 & $10.0 \pm 0.7$ & -0.77 \\
\hline 3777.662 & 28337.814 & ev & 2.5 & 1873.934 & od & 3.5 & $5.8 \pm 0.6$ & -1.13 \\
\hline 3781.616 & 30702.610 & ev & 4.5 & 4266.397 & od & 3.5 & $25.6 \pm 1.4$ & -0.26 \\
\hline 3786.632 & 27811.496 & ev & 3.5 & 1410.304 & od & 4.5 & $25.1 \pm 1.3$ & -0.36 \\
\hline 3787.903 & 27379.949 & ev & 5.5 & 987.611 & od & 4.5 & $4.03 \pm 0.28$ & -0.98 \\
\hline 3788.746 & 30180.096 & ev & 6.5 & 3793.634 & od & 6.5 & $16.5 \pm 1.1$ & -0.30 \\
\hline 3791.002 & 30637.157 & ev & 2.5 & 4266.397 & od & 3.5 & $1.48 \pm 0.15$ & -1.72 \\
\hline 3791.219 & 30829.124 & ev & 3.5 & 4459.872 & od & 3.5 & $1.43 \pm 0.15$ & -1.61 \\
\hline 3792.324 & 30065.164 & ev & 3.5 & 3703.594 & od & 3.5 & $18.1 \pm 1.2$ & -0.51 \\
\hline 3794.210 & 28730.712 & ev & 3.5 & 2382.246 & od & 4.5 & $1.48 \pm 0.12$ & -1.59 \\
\hline 3795.011 & 28725.148 & ev & 4.5 & 2382.246 & od & 4.5 & $5.9 \pm 0.4$ & -0.89 \\
\hline 3795.246 & 30134.910 & ev & 5.5 & 3793.634 & od & 6.5 & $6.7 \pm 0.5$ & -0.76 \\
\hline 3799.032 & 29908.904 & ev & 4.5 & 3593.882 & od & 4.5 & $2.51 \pm 0.16$ & -1.26 \\
\hline 3800.322 & 30829.124 & ev & 3.5 & 4523.033 & od & 4.5 & $8.2 \pm 0.6$ & -0.85 \\
\hline 3801.526 & 33531.388 & ev & 6.5 & 7233.627 & od & 5.5 & $139 \pm 7$ & 0.63 \\
\hline 3803.096 & 29166.597 & ev & 4.5 & 2879.695 & od & 5.5 & $24.9 \pm 1.7$ & -0.27 \\
\hline 3808.113 & 28634.516 & ev & 5.5 & 2382.246 & od & 4.5 & $27.7 \pm 1.6$ & -0.14 \\
\hline 3808.382 & 30245.878 & ev & 4.5 & 3995.460 & od & 3.5 & $1.33 \pm 0.12$ & -1.54 \\
\hline 3809.218 & 31155.623 & ev & 6.5 & 4910.963 & od & 5.5 & $20.3 \pm 1.3$ & -0.21 \\
\hline 3809.497 & 30702.610 & ev & 4.5 & 4459.872 & od & 3.5 & $4.6 \pm 0.6$ & -1.00 \\
\hline 3814.938 & 29908.904 & ev & 4.5 & 3703.594 & od & 3.5 & $2.15 \pm 0.16$ & -1.33 \\
\hline 3815.793 & 27187.047 & ev & 3.5 & 987.611 & od & 4.5 & $15.1 \pm 1.1$ & -0.58 \\
\hline 3818.688 & 30702.610 & ev & 4.5 & 4523.033 & od & 4.5 & $4.19 \pm 0.30$ & -1.04 \\
\hline 3819.022 & 30637.157 & ev & 2.5 & 4459.872 & od & 3.5 & $27.2 \pm 2.6$ & -0.45 \\
\hline 3819.998 & 30166.057 & ev & 3.5 & 3995.460 & od & 3.5 & $2.08 \pm 0.17$ & -1.44 \\
\hline 3821.266 & 28725.148 & ev & 4.5 & 2563.233 & od & 5.5 & $7.6 \pm 0.4$ & -0.78 \\
\hline 3823.900 & 28725.148 & ev & 4.5 & 2581.257 & od & 4.5 & $15.1 \pm 1.1$ & -0.48 \\
\hline 3826.534 & 30637.157 & ev & 2.5 & 4511.257 & od & 2.5 & $2.7 \pm 0.5$ & -1.44 \\
\hline 3829.820 & 29807.078 & ev & 3.5 & 3703.594 & od & 3.5 & $1.73 \pm 0.21$ & -1.52 \\
\hline 3830.023 & 32492.038 & ev & 5.5 & 6389.942 & od & 4.5 & $8.3 \pm 0.5$ & -0.66 \\
\hline 3830.911 & 28730.712 & ev & 3.5 & 2634.666 & od & 2.5 & $9.3 \pm 0.6$ & -0.79 \\
\hline 3831.542 & 30829.124 & ev & 3.5 & 4737.373 & od & 2.5 & $6.0 \pm 1.0$ & -0.98 \\
\hline 3831.663 & 29794.517 & ev & 3.5 & 3703.594 & od & 3.5 & $4.1 \pm 0.4$ & -1.15 \\
\hline 3831.782 & 28685.758 & ev & 2.5 & 2595.644 & od & 1.5 & $4.7 \pm 0.4$ & -1.21 \\
\hline 3832.221 & 31738.484 & ev & 5.5 & 5651.357 & od & 5.5 & $8.1 \pm 0.7$ & -0.67 \\
\hline 3832.335 & 29449.778 & ev & 1.5 & 3363.427 & od & 2.5 & $3.88 \pm 0.27$ & -1.47 \\
\hline 3832.741 & 28725.148 & ev & 4.5 & 2641.559 & od & 3.5 & $3.9 \pm 0.4$ & -1.07 \\
\hline 3834.550 & 28634.516 & ev & 5.5 & 2563.233 & od & 5.5 & $12.4 \pm 0.8$ & -0.49 \\
\hline 3834.782 & 30065.164 & ev & 3.5 & 3995.460 & od & 3.5 & $7.9 \pm 0.5$ & -0.86 \\
\hline 3836.107 & 27934.638 & ev & 4.5 & 1873.934 & od & 3.5 & $8.5 \pm 0.5$ & -0.73 \\
\hline 3837.203 & 28634.516 & ev & 5.5 & 2581.257 & od & 4.5 & $3.70 \pm 0.28$ & -1.01 \\
\hline 3837.522 & 28685.758 & ev & 2.5 & 2634.666 & od & 2.5 & $1.30 \pm 0.10$ & -1.76 \\
\hline 3838.538 & 28685.758 & ev & 2.5 & 2641.559 & od & 3.5 & $55.0 \pm 3.0$ & -0.14 \\
\hline 3843.767 & 33531.388 & ev & 6.5 & 7522.622 & od & 5.5 & $14.0 \pm 1.3$ & -0.36 \\
\hline 3845.273 & 29994.041 & ev & 2.5 & 3995.460 & od & 3.5 & $4.2 \pm 0.5$ & -1.25 \\
\hline 3848.100 & 30245.878 & ev & 4.5 & 4266.397 & od & 3.5 & $13.1 \pm 0.9$ & -0.54 \\
\hline 3848.592 & 30180.096 & ev & 6.5 & 4203.934 & od & 6.5 & $27.4 \pm 1.6$ & -0.07 \\
\hline 3849.558 & 27379.949 & ev & 5.5 & 1410.304 & od & 4.5 & $3.7 \pm 0.3$ & -1.01 \\
\hline 3852.099 & 28334.756 & ev & 4.5 & 2382.246 & od & 4.5 & $3.7 \pm 0.3$ & -1.08 \\
\hline 3853.156 & 25945.396 & ev & 3.5 & 0.000 & od & 3.5 & $17.5 \pm 1.0$ & -0.51 \\
\hline 3854.185 & 27812.398 & ev & 2.5 & 1873.934 & od & 3.5 & $43.1 \pm 2.3$ & -0.24 \\
\hline 3854.319 & 27811.496 & ev & 3.5 & 1873.934 & od & 3.5 & $39.7 \pm 2.1$ & -0.15 \\
\hline 3855.298 & 30134.910 & ev & 5.5 & 4203.934 & od & 6.5 & $19.5 \pm 1.2$ & -0.28 \\
\hline 3857.025 & 31738.484 & ev & 5.5 & 5819.113 & od & 4.5 & $21.0 \pm 1.9$ & -0.25 \\
\hline 3857.237 & 29281.374 & ev & 2.5 & 3363.427 & od & 2.5 & $13.0 \pm 0.9$ & -0.76 \\
\hline 3857.641 & 28297.473 & ev & 3.5 & 2382.246 & od & 4.5 & $13.0 \pm 0.8$ & -0.63 \\
\hline
\end{tabular}


Table 2

(Continued)

\begin{tabular}{|c|c|c|c|c|c|c|c|c|}
\hline$\lambda_{\text {air }}(\AA)$ & $E_{\text {upper }}\left(\mathrm{cm}^{-1}\right)$ & Parity & $J_{\text {upp }}$ & $E_{\text {lower }}\left(\mathrm{cm}^{-1}\right)$ & Parity & $J_{\text {low }}$ & $A$-value $\left(10^{6} \mathrm{~s}^{-1}\right)$ & $\log (g f)$ \\
\hline 3868.133 & 29438.817 & ev & 5.5 & 3593.882 & od & 4.5 & $5.7 \pm 0.3$ & -0.81 \\
\hline 3870.867 & 31340.393 & od & 6.5 & 5513.709 & ev & 5.5 & $5.2 \pm 0.4$ & -0.79 \\
\hline 3872.131 & 30829.124 & ev & 3.5 & 5010.870 & od & 2.5 & $2.70 \pm 0.19$ & -1.31 \\
\hline 3873.126 & 29807.078 & ev & 3.5 & 3995.460 & od & 3.5 & $1.55 \pm 0.11$ & -1.55 \\
\hline 3875.012 & 29794.517 & ev & 3.5 & 3995.460 & od & 3.5 & $8.0 \pm 0.7$ & -0.84 \\
\hline 3875.056 & 30065.164 & ev & 3.5 & 4266.397 & od & 3.5 & $10.2 \pm 0.7$ & -0.74 \\
\hline 3875.995 & 30637.157 & ev & 2.5 & 4844.644 & od & 1.5 & $2.24 \pm 0.18$ & -1.52 \\
\hline 3876.126 & 30702.610 & ev & 4.5 & 4910.963 & od & 5.5 & $11.7 \pm 0.8$ & -0.58 \\
\hline 3878.367 & 27187.047 & ev & 3.5 & 1410.304 & od & 4.5 & $24.9 \pm 1.5$ & -0.35 \\
\hline 3879.460 & 31738.484 & ev & 5.5 & 5969.007 & od & 5.5 & $0.60 \pm 0.08$ & -1.79 \\
\hline 3881.668 & 28634.516 & ev & 5.5 & 2879.695 & od & 5.5 & $6.7 \pm 0.5$ & -0.74 \\
\hline 3881.867 & 28334.756 & ev & 4.5 & 2581.257 & od & 4.5 & $7.1 \pm 1.4$ & -0.79 \\
\hline 3882.445 & 28345.313 & ev & 0.5 & 2595.644 & od & 1.5 & $144 \pm 7$ & -0.19 \\
\hline 3883.437 & 32802.165 & ev & 5.5 & 7059.072 & od & 4.5 & $2.08 \pm 0.23$ & -1.25 \\
\hline 3883.533 & 30065.164 & $\mathrm{ev}$ & 3.5 & 4322.708 & od & 2.5 & $4.7 \pm 0.4$ & -1.07 \\
\hline 3883.576 & 28337.814 & ev & 2.5 & 2595.644 & od & 1.5 & $6.7 \pm 0.5$ & -1.04 \\
\hline 3885.769 & 29994.041 & ev & 2.5 & 4266.397 & od & 3.5 & $3.37 \pm 0.25$ & -1.34 \\
\hline 3886.493 & 30245.878 & $\mathrm{ev}$ & 4.5 & 4523.033 & od & 4.5 & $4.0 \pm 0.4$ & -1.05 \\
\hline 3888.387 & 30829.124 & ev & 3.5 & 5118.806 & od & 2.5 & $8.6 \pm 0.6$ & -0.80 \\
\hline 3889.297 & 29449.778 & ev & 1.5 & 3745.475 & od & 1.5 & $7.6 \pm 0.6$ & -1.16 \\
\hline 3889.472 & 28337.814 & ev & 2.5 & 2634.666 & od & 2.5 & $3.7 \pm 0.4$ & -1.29 \\
\hline 3889.982 & 31155.623 & ev & 6.5 & 5455.845 & od & 7.5 & $41.9 \pm 2.3$ & 0.12 \\
\hline 3890.515 & 28337.814 & ev & 2.5 & 2641.559 & od & 3.5 & $3.01 \pm 0.30$ & -1.39 \\
\hline 3890.744 & 27835.233 & ev & 1.5 & 2140.492 & od & 0.5 & $18.4 \pm 1.3$ & -0.78 \\
\hline 3890.978 & 28334.756 & ev & 4.5 & 2641.559 & od & 3.5 & $7.3 \pm 0.5$ & -0.78 \\
\hline 3894.292 & 29994.041 & ev & 2.5 & 4322.708 & od & 2.5 & $3.63 \pm 0.21$ & -1.31 \\
\hline 3896.633 & 28297.473 & $\mathrm{ev}$ & 3.5 & 2641.559 & od & 3.5 & $1.41 \pm 0.10$ & -1.59 \\
\hline 3896.802 & 30166.057 & ev & 3.5 & 4511.257 & od & 2.5 & $29.5 \pm 1.6$ & -0.27 \\
\hline 3898.263 & 29438.817 & ev & 5.5 & 3793.634 & od & 6.5 & $16.7 \pm 1.0$ & -0.34 \\
\hline 3898.670 & 29908.904 & $\mathrm{ev}$ & 4.5 & 4266.397 & od & 3.5 & $1.28 \pm 0.08$ & -1.53 \\
\hline 3903.333 & 30134.910 & ev & 5.5 & 4523.033 & od & 4.5 & $6.7 \pm 0.4$ & -0.73 \\
\hline 3904.337 & 30065.164 & ev & 3.5 & 4459.872 & od & 3.5 & $14.7 \pm 1.1$ & -0.57 \\
\hline 3907.434 & 29750.547 & od & 5.5 & 4165.550 & ev & 4.5 & $6.5 \pm 0.4$ & -0.75 \\
\hline 3908.404 & 32492.038 & ev & 5.5 & 6913.392 & od & 6.5 & $42.4 \pm 2.5$ & 0.07 \\
\hline 3908.536 & 29281.374 & ev & 2.5 & 3703.594 & od & 3.5 & $23.9 \pm 1.3$ & -0.48 \\
\hline 3909.310 & 29166.597 & ev & 4.5 & 3593.882 & od & 4.5 & $10.0 \pm 0.6$ & -0.64 \\
\hline 3909.747 & 29735.413 & od & 4.5 & 4165.550 & ev & 4.5 & $4.78 \pm 0.29$ & -0.96 \\
\hline 3912.188 & 30065.164 & ev & 3.5 & 4511.257 & od & 2.5 & $15.1 \pm 0.9$ & -0.56 \\
\hline 3912.420 & 27934.638 & $\mathrm{ev}$ & 4.5 & 2382.246 & od & 4.5 & $24.7 \pm 1.3$ & -0.25 \\
\hline 3913.992 & 30065.164 & ev & 3.5 & 4523.033 & od & 4.5 & $6.6 \pm 0.4$ & -0.92 \\
\hline 3914.947 & 29281.374 & ev & 2.5 & 3745.475 & od & 1.5 & $5.1 \pm 0.3$ & -1.16 \\
\hline 3916.140 & 29794.517 & $\mathrm{ev}$ & 3.5 & 4266.397 & od & 3.5 & $11.9 \pm 0.7$ & -0.66 \\
\hline 3917.639 & 30637.157 & ev & 2.5 & 5118.806 & od & 2.5 & $19.9 \pm 1.3$ & -0.56 \\
\hline 3919.803 & 31155.623 & ev & 6.5 & 5651.357 & od & 5.5 & $22.4 \pm 1.5$ & -0.14 \\
\hline 3921.989 & 26900.354 & ev & 3.5 & 1410.304 & od & 4.5 & $0.21 \pm 0.04$ & -2.42 \\
\hline 3922.863 & 29807.078 & ev & 3.5 & 4322.708 & od & 2.5 & $5.7 \pm 0.6$ & -0.98 \\
\hline 3923.107 & 29994.041 & ev & 2.5 & 4511.257 & od & 2.5 & $44.2 \pm 2.5$ & -0.21 \\
\hline 3927.380 & 28334.756 & ev & 4.5 & 2879.695 & od & 5.5 & $3.4 \pm 0.3$ & -1.11 \\
\hline 3928.310 & 29908.904 & ev & 4.5 & 4459.872 & od & 3.5 & $5.9 \pm 0.4$ & -0.86 \\
\hline 3930.792 & 32492.038 & ev & 5.5 & 7059.072 & od & 4.5 & $9.7 \pm 0.7$ & -0.57 \\
\hline 3931.083 & 26841.384 & $\mathrm{ev}$ & 4.5 & 1410.304 & od & 4.5 & $14.6 \pm 0.9$ & -0.47 \\
\hline 3931.366 & 27811.496 & ev & 3.5 & 2382.246 & od & 4.5 & $15.4 \pm 0.9$ & -0.54 \\
\hline 3937.180 & 30829.124 & $\mathrm{ev}$ & 3.5 & 5437.422 & od & 3.5 & $0.80 \pm 0.08$ & -1.83 \\
\hline 3939.657 & 27249.669 & ev & 2.5 & 1873.934 & od & 3.5 & $2.34 \pm 0.15$ & -1.49 \\
\hline 3940.330 & 27934.638 & ev & 4.5 & 2563.233 & od & 5.5 & $23.3 \pm 1.2$ & -0.27 \\
\hline 3940.970 & 28730.712 & ev & 3.5 & 3363.427 & od & 2.5 & $14.6 \pm 0.9$ & -0.57 \\
\hline 3942.151 & 25359.686 & ev & 2.5 & 0.000 & od & 3.5 & $43.1 \pm 2.4$ & -0.22 \\
\hline 3942.745 & 32269.252 & ev & 7.5 & 6913.392 & od & 6.5 & $132 \pm 7$ & 0.69 \\
\hline 3943.131 & 27934.638 & ev & 4.5 & 2581.257 & od & 4.5 & $7.3 \pm 0.4$ & -0.77 \\
\hline 3943.884 & 31738.484 & ev & 5.5 & 6389.942 & od & 4.5 & $55 \pm 3$ & 0.19 \\
\hline 3944.092 & 29807.078 & ev & 3.5 & 4459.872 & od & 3.5 & $4.8 \pm 0.3$ & -1.05 \\
\hline 3946.047 & 29794.517 & ev & 3.5 & 4459.872 & od & 3.5 & $0.39 \pm 0.04$ & -2.14 \\
\hline 3947.115 & 30065.164 & ev & 3.5 & 4737.373 & od & 2.5 & $1.00 \pm 0.10$ & -1.73 \\
\hline 3947.966 & 28685.758 & ev & 2.5 & 3363.427 & od & 2.5 & $12.9 \pm 0.8$ & -0.74 \\
\hline 3949.404 & 27187.047 & ev & 3.5 & 1873.934 & od & 3.5 & $5.0 \pm 0.3$ & -1.03 \\
\hline 3952.104 & 29807.078 & ev & 3.5 & 4511.257 & od & 2.5 & $5.7 \pm 0.5$ & -0.97 \\
\hline
\end{tabular}


Table 2

(Continued)

\begin{tabular}{|c|c|c|c|c|c|c|c|c|}
\hline$\lambda_{\text {air }}(\AA)$ & $E_{\text {upper }}\left(\mathrm{cm}^{-1}\right)$ & Parity & $J_{\text {upp }}$ & $E_{\text {lower }}\left(\mathrm{cm}^{-1}\right)$ & Parity & $J_{\text {low }}$ & $A$-value $\left(10^{6} \mathrm{~s}^{-1}\right)$ & $\log (g f)$ \\
\hline 3952.532 & 27934.638 & ev & 4.5 & 2641.559 & od & 3.5 & $33.5 \pm 1.7$ & -0.11 \\
\hline 3953.652 & 29281.374 & ev & 2.5 & 3995.460 & od & 3.5 & $16.4 \pm 0.9$ & -0.64 \\
\hline 3953.944 & 29807.078 & ev & 3.5 & 4523.033 & od & 4.5 & $5.3 \pm 0.4$ & -1.00 \\
\hline 3955.910 & 29794.517 & ev & 3.5 & 4523.033 & od & 4.5 & $8.7 \pm 0.8$ & -0.79 \\
\hline 3956.278 & 30180.096 & ev & 6.5 & 4910.963 & od & 5.5 & $39.8 \pm 2.1$ & 0.12 \\
\hline 3956.896 & 30702.610 & ev & 4.5 & 5437.422 & od & 3.5 & $10.4 \pm 0.6$ & -0.61 \\
\hline 3957.957 & 32492.038 & ev & 5.5 & 7233.627 & od & 5.5 & $10.3 \pm 1.2$ & -0.54 \\
\hline 3959.607 & 29449.778 & ev & 1.5 & 4201.893 & od & 1.5 & $16.6 \pm 1.4$ & -0.81 \\
\hline 3960.909 & 27835.233 & ev & 1.5 & 2595.644 & od & 1.5 & $45.9 \pm 2.4$ & -0.36 \\
\hline 3961.648 & 29438.817 & ev & 5.5 & 4203.934 & od & 6.5 & $1.45 \pm 0.14$ & -1.39 \\
\hline 3963.365 & 30134.910 & ev & 5.5 & 4910.963 & od & 5.5 & $2.86 \pm 0.23$ & -1.09 \\
\hline 3964.496 & 27812.398 & ev & 2.5 & 2595.644 & od & 1.5 & $15.7 \pm 0.9$ & -0.65 \\
\hline 3967.042 & 27835.233 & ev & 1.5 & 2634.666 & od & 2.5 & $47.4 \pm 2.4$ & -0.35 \\
\hline 3967.173 & 30637.157 & ev & 2.5 & 5437.422 & od & 3.5 & $18.5 \pm 1.2$ & -0.58 \\
\hline 3967.431 & 32492.038 & ev & 5.5 & 7293.938 & od & 6.5 & $0.85 \pm 0.08$ & -1.62 \\
\hline 3969.240 & 31155.623 & ev & 6.5 & 5969.007 & od & 5.5 & $2.2 \pm 0.3$ & -1.14 \\
\hline 3970.640 & 27812.398 & ev & 2.5 & 2634.666 & od & 2.5 & $7.5 \pm 0.5$ & -0.97 \\
\hline 3970.783 & 27811.496 & ev & 3.5 & 2634.666 & od & 2.5 & $0.61 \pm 0.04$ & -1.94 \\
\hline 3971.681 & 29166.597 & ev & 4.5 & 3995.460 & od & 3.5 & $16.4 \pm 0.9$ & -0.41 \\
\hline 3971.870 & 27811.496 & ev & 3.5 & 2641.559 & od & 3.5 & $4.3 \pm 0.4$ & -1.09 \\
\hline 3974.199 & 30166.057 & ev & 3.5 & 5010.870 & od & 2.5 & $3.6 \pm 0.3$ & -1.16 \\
\hline 3974.488 & 30829.124 & ev & 3.5 & 5675.763 & od & 4.5 & $1.07 \pm 0.11$ & -1.69 \\
\hline 3978.646 & 29449.778 & ev & 1.5 & 4322.708 & od & 2.5 & $60 \pm 3$ & -0.24 \\
\hline 3980.890 & 30829.124 & ev & 3.5 & 5716.216 & od & 3.5 & $32.2 \pm 2.1$ & -0.21 \\
\hline 3982.901 & 31738.484 & ev & 5.5 & 6638.258 & od & 4.5 & $18.1 \pm 2.5$ & -0.29 \\
\hline 3983.288 & 29263.338 & od & 5.5 & 4165.550 & ev & 4.5 & $8.9 \pm 0.6$ & -0.59 \\
\hline 3984.671 & 32802.165 & ev & 5.5 & 7713.089 & od & 4.5 & $34.7 \pm 2.8$ & 0.00 \\
\hline 3990.100 & 27934.638 & ev & 4.5 & 2879.695 & od & 5.5 & $4.86 \pm 0.27$ & -0.94 \\
\hline 3990.688 & 30702.610 & ev & 4.5 & 5651.357 & od & 5.5 & $4.2 \pm 0.4$ & -0.99 \\
\hline 3991.325 & 30166.057 & ev & 3.5 & 5118.806 & od & 2.5 & $2.30 \pm 0.13$ & -1.36 \\
\hline 3992.380 & 28634.516 & ev & 5.5 & 3593.882 & od & 4.5 & $20.8 \pm 1.1$ & -0.22 \\
\hline 3993.819 & 32372.621 & od & 4.5 & 7341.007 & ev & 5.5 & $81 \pm 4$ & 0.29 \\
\hline 3994.580 & 30702.610 & ev & 4.5 & 5675.763 & od & 4.5 & $3.40 \pm 0.20$ & -1.09 \\
\hline 3994.648 & 26900.354 & ev & 3.5 & 1873.934 & od & 3.5 & $0.49 \pm 0.06$ & -2.02 \\
\hline 3995.425 & 28725.148 & ev & 4.5 & 3703.594 & od & 3.5 & $0.97 \pm 0.07$ & -1.63 \\
\hline 3996.475 & 29281.374 & ev & 2.5 & 4266.397 & od & 3.5 & $4.5 \pm 0.3$ & -1.19 \\
\hline 3997.269 & 30829.124 & ev & 3.5 & 5819.113 & od & 4.5 & $1.18 \pm 0.10$ & -1.64 \\
\hline 3999.237 & 27379.949 & ev & 5.5 & 2382.246 & od & 4.5 & $39.6 \pm 2.0$ & 0.06 \\
\hline 4001.047 & 30702.610 & ev & 4.5 & 5716.216 & od & 3.5 & $7.6 \pm 0.6$ & -0.74 \\
\hline 4001.563 & 29994.041 & ev & 2.5 & 5010.870 & od & 2.5 & $17.9 \pm 1.4$ & -0.59 \\
\hline 4001.724 & 28685.758 & ev & 2.5 & 3703.594 & od & 3.5 & $10.3 \pm 0.7$ & -0.83 \\
\hline 4002.822 & 32269.252 & ev & 7.5 & 7293.938 & od & 6.5 & $11.4 \pm 0.8$ & -0.36 \\
\hline 4002.971 & 28337.814 & ev & 2.5 & 3363.427 & od & 2.5 & $5.7 \pm 0.5$ & -1.09 \\
\hline 4003.767 & 32492.038 & ev & 5.5 & 7522.622 & od & 5.5 & $68 \pm 4$ & 0.29 \\
\hline 4004.083 & 26841.384 & ev & 4.5 & 1873.934 & od & 3.5 & $0.63 \pm 0.08$ & -1.82 \\
\hline 4005.492 & 29281.374 & ev & 2.5 & 4322.708 & od & 2.5 & $0.43 \pm 0.05$ & -2.20 \\
\hline 4005.633 & 25945.396 & ev & 3.5 & 987.611 & od & 4.5 & $9.8 \pm 0.6$ & -0.73 \\
\hline 4008.444 & 28685.758 & ev & 2.5 & 3745.475 & od & 1.5 & $1.70 \pm 0.14$ & -1.61 \\
\hline 4011.556 & 30637.157 & ev & 2.5 & 5716.216 & od & 3.5 & $6.1 \pm 0.7$ & -1.05 \\
\hline 4012.386 & 29438.817 & ev & 5.5 & 4523.033 & od & 4.5 & $67 \pm 3$ & 0.29 \\
\hline 4014.897 & 29166.597 & ev & 4.5 & 4266.397 & od & 3.5 & $25.9 \pm 1.4$ & -0.20 \\
\hline 4017.135 & 30829.124 & ev & 3.5 & 5942.798 & od & 3.5 & $1.54 \pm 0.14$ & -1.53 \\
\hline 4017.592 & 30702.610 & ev & 4.5 & 5819.113 & od & 4.5 & $4.12 \pm 0.24$ & -1.00 \\
\hline 4024.485 & 28634.516 & ev & 5.5 & 3793.634 & od & 6.5 & $18.6 \pm 1.0$ & -0.27 \\
\hline 4025.139 & 28345.313 & ev & 0.5 & 3508.470 & od & 0.5 & $34.5 \pm 2.0$ & -0.78 \\
\hline 4027.044 & 31738.484 & ev & 5.5 & 6913.392 & od & 6.5 & $2.43 \pm 0.23$ & -1.15 \\
\hline 4027.627 & 29281.374 & ev & 2.5 & 4459.872 & od & 3.5 & $4.6 \pm 0.6$ & -1.17 \\
\hline 4028.404 & 27379.949 & ev & 5.5 & 2563.233 & od & 5.5 & $18.5 \pm 1.0$ & -0.27 \\
\hline 4031.332 & 27379.949 & ev & 5.5 & 2581.257 & od & 4.5 & $19.7 \pm 1.1$ & -0.24 \\
\hline 4033.779 & 29794.517 & ev & 3.5 & 5010.870 & od & 2.5 & $1.34 \pm 0.09$ & -1.58 \\
\hline 4035.982 & 29281.374 & ev & 2.5 & 4511.257 & od & 2.5 & $0.47 \pm 0.06$ & -2.17 \\
\hline 4037.662 & 30702.610 & ev & 4.5 & 5942.798 & od & 3.5 & $14.3 \pm 1.0$ & -0.46 \\
\hline 4040.753 & 28334.756 & ev & 4.5 & 3593.882 & od & 4.5 & $66 \pm 3$ & 0.21 \\
\hline 4041.269 & 30702.610 & ev & 4.5 & 5964.896 & od & 3.5 & $6.8 \pm 0.5$ & -0.78 \\
\hline 4042.581 & 28725.148 & ev & 4.5 & 3995.460 & od & 3.5 & $41.1 \pm 2.1$ & 0.00 \\
\hline 4045.318 & 30637.157 & ev & 2.5 & 5924.204 & od & 1.5 & $7.6 \pm 0.5$ & -0.95 \\
\hline
\end{tabular}


Table 2

(Continued)

\begin{tabular}{|c|c|c|c|c|c|c|c|c|}
\hline$\lambda_{\text {air }}(\AA)$ & $E_{\text {upper }}\left(\mathrm{cm}^{-1}\right)$ & Parity & $J_{\text {upp }}$ & $E_{\text {lower }}\left(\mathrm{cm}^{-1}\right)$ & Parity & $J_{\text {low }}$ & $A$-value $\left(10^{6} \mathrm{~s}^{-1}\right)$ & $\log (g f)$ \\
\hline 4045.408 & 29449.778 & ev & 1.5 & 4737.373 & od & 2.5 & $1.73 \pm 0.15$ & -1.77 \\
\hline 4046.338 & 29166.597 & ev & 4.5 & 4459.872 & od & 3.5 & $28.5 \pm 1.6$ & -0.16 \\
\hline 4046.851 & 28297.473 & ev & 3.5 & 3593.882 & od & 4.5 & $0.67 \pm 0.08$ & -1.88 \\
\hline 4048.364 & 30637.157 & ev & 2.5 & 5942.798 & od & 3.5 & $3.03 \pm 0.23$ & -1.35 \\
\hline 4049.362 & 29807.078 & ev & 3.5 & 5118.806 & od & 2.5 & $2.5 \pm 0.3$ & -1.31 \\
\hline 4051.424 & 29794.517 & ev & 3.5 & 5118.806 & od & 2.5 & $9.5 \pm 0.8$ & -0.73 \\
\hline 4051.990 & 30637.157 & ev & 2.5 & 5964.896 & od & 3.5 & $11.9 \pm 1.1$ & -0.76 \\
\hline 4053.503 & 24663.053 & ev & 4.5 & 0.000 & od & 3.5 & $10.0 \pm 0.5$ & -0.61 \\
\hline 4058.248 & 28337.814 & ev & 2.5 & 3703.594 & od & 3.5 & $4.86 \pm 0.30$ & -1.14 \\
\hline 4058.751 & 28334.756 & ev & 4.5 & 3703.594 & od & 3.5 & $2.29 \pm 0.22$ & -1.25 \\
\hline 4061.416 & 27249.669 & ev & 2.5 & 2634.666 & od & 2.5 & $1.43 \pm 0.23$ & -1.67 \\
\hline 4062.937 & 27187.047 & ev & 3.5 & 2581.257 & od & 4.5 & $8.4 \pm 0.5$ & -0.78 \\
\hline 4063.045 & 29449.778 & ev & 1.5 & 4844.644 & od & 1.5 & $1.89 \pm 0.21$ & -1.73 \\
\hline 4063.920 & 28345.313 & ev & 0.5 & 3745.475 & od & 1.5 & $16.8 \pm 1.0$ & -1.08 \\
\hline 4064.798 & 30245.878 & ev & 4.5 & 5651.357 & od & 5.5 & $0.58 \pm 0.04$ & -1.84 \\
\hline 4064.904 & 28297.473 & ev & 3.5 & 3703.594 & od & 3.5 & $3.23 \pm 0.21$ & -1.19 \\
\hline 4068.836 & 30245.878 & ev & 4.5 & 5675.763 & od & 4.5 & $27.3 \pm 1.5$ & -0.17 \\
\hline 4071.072 & 29994.041 & ev & 2.5 & 5437.422 & od & 3.5 & $6.6 \pm 0.7$ & -1.00 \\
\hline 4071.775 & 27187.047 & ev & 3.5 & 2634.666 & od & 2.5 & $31.5 \pm 1.6$ & -0.20 \\
\hline 4072.918 & 27187.047 & ev & 3.5 & 2641.559 & od & 3.5 & $11.5 \pm 0.6$ & -0.64 \\
\hline 4073.474 & 28396.150 & od & 2.5 & 3854.012 & ev & 3.5 & $109 \pm 6$ & 0.21 \\
\hline 4074.644 & 25945.396 & ev & 3.5 & 1410.304 & od & 4.5 & $1.00 \pm 0.09$ & -1.70 \\
\hline 4075.546 & 30245.878 & ev & 4.5 & 5716.216 & od & 3.5 & $1.80 \pm 0.20$ & -1.35 \\
\hline 4075.700 & 30180.096 & ev & 6.5 & 5651.357 & od & 5.5 & $48.7 \pm 2.5$ & 0.23 \\
\hline 4075.847 & 29438.817 & ev & 5.5 & 4910.963 & od & 5.5 & $48.6 \pm 2.6$ & 0.16 \\
\hline 4079.672 & 31738.484 & ev & 5.5 & 7233.627 & od & 5.5 & $13.2 \pm 1.1$ & -0.40 \\
\hline 4080.438 & 27379.949 & ev & 5.5 & 2879.695 & od & 5.5 & $6.7 \pm 0.4$ & -0.70 \\
\hline 4082.098 & 30166.057 & ev & 3.5 & 5675.763 & od & 4.5 & $1.04 \pm 0.11$ & -1.68 \\
\hline 4083.222 & 30134.910 & ev & 5.5 & 5651.357 & od & 5.5 & $62 \pm 3$ & 0.27 \\
\hline 4085.236 & 29908.904 & ev & 4.5 & 5437.422 & od & 3.5 & $28.1 \pm 1.8$ & -0.15 \\
\hline 4086.433 & 28730.712 & ev & 3.5 & 4266.397 & od & 3.5 & $5.3 \pm 0.6$ & -0.97 \\
\hline 4087.362 & 28725.148 & ev & 4.5 & 4266.397 & od & 3.5 & $7.5 \pm 0.4$ & -0.73 \\
\hline 4088.852 & 30166.057 & ev & 3.5 & 5716.216 & od & 3.5 & $17.5 \pm 1.1$ & -0.46 \\
\hline 4088.997 & 27812.398 & ev & 2.5 & 3363.427 & od & 2.5 & $4.62 \pm 0.27$ & -1.16 \\
\hline 4089.148 & 27811.496 & ev & 3.5 & 3363.427 & od & 2.5 & $1.38 \pm 0.11$ & -1.56 \\
\hline 4089.738 & 31738.484 & ev & 5.5 & 7293.938 & od & 6.5 & $6.1 \pm 0.6$ & -0.74 \\
\hline 4091.046 & 29281.374 & ev & 2.5 & 4844.644 & od & 1.5 & $2.70 \pm 0.23$ & -1.39 \\
\hline 4092.075 & 28634.516 & ev & 5.5 & 4203.934 & od & 6.5 & $2.17 \pm 0.25$ & -1.18 \\
\hline 4092.715 & 30245.878 & ev & 4.5 & 5819.113 & od & 4.5 & $6.3 \pm 0.4$ & -0.80 \\
\hline 4093.956 & 28685.758 & ev & 2.5 & 4266.397 & od & 3.5 & $11.7 \pm 0.8$ & -0.75 \\
\hline 4098.985 & 30065.164 & ev & 3.5 & 5675.763 & od & 4.5 & $9.0 \pm 0.7$ & -0.74 \\
\hline 4101.769 & 31340.393 & od & 6.5 & 6967.547 & ev & 6.5 & $31.4 \pm 1.7$ & 0.05 \\
\hline 4106.133 & 30166.057 & ev & 3.5 & 5819.113 & od & 4.5 & $6.6 \pm 0.4$ & -0.87 \\
\hline 4106.907 & 28337.814 & ev & 2.5 & 3995.460 & od & 3.5 & $5.3 \pm 0.3$ & -1.10 \\
\hline 4107.177 & 27934.638 & ev & 4.5 & 3593.882 & od & 4.5 & $0.35 \pm 0.06$ & -2.05 \\
\hline 4107.423 & 28334.756 & ev & 4.5 & 3995.460 & od & 3.5 & $28.9 \pm 1.5$ & -0.14 \\
\hline 4108.828 & 29449.778 & ev & 1.5 & 5118.806 & od & 2.5 & $0.40 \pm 0.06$ & -2.39 \\
\hline 4109.539 & 27835.233 & ev & 1.5 & 3508.470 & od & 0.5 & $5.1 \pm 0.4$ & -1.28 \\
\hline 4110.835 & 26900.354 & ev & 3.5 & 2581.257 & od & 4.5 & $4.07 \pm 0.28$ & -1.08 \\
\hline 4111.393 & 30134.910 & ev & 5.5 & 5819.113 & od & 4.5 & $14.6 \pm 1.0$ & -0.35 \\
\hline 4111.922 & 30702.610 & ev & 4.5 & 6389.942 & od & 4.5 & $4.35 \pm 0.27$ & -0.96 \\
\hline 4113.544 & 30245.878 & ev & 4.5 & 5942.798 & od & 3.5 & $1.91 \pm 0.20$ & -1.32 \\
\hline 4113.725 & 28297.473 & ev & 3.5 & 3995.460 & od & 3.5 & $7.1 \pm 0.5$ & -0.84 \\
\hline 4117.288 & 30245.878 & ev & 4.5 & 5964.896 & od & 3.5 & $13.9 \pm 1.0$ & -0.45 \\
\hline 4117.768 & 26841.384 & ev & 4.5 & 2563.233 & od & 5.5 & $0.23 \pm 0.04$ & -2.23 \\
\hline 4117.823 & 29994.041 & ev & 2.5 & 5716.216 & od & 3.5 & $3.4 \pm 0.4$ & -1.28 \\
\hline 4117.985 & 30245.878 & ev & 4.5 & 5969.007 & od & 5.5 & $4.3 \pm 0.4$ & -0.96 \\
\hline 4118.143 & 29892.677 & od & 3.5 & 5616.739 & ev & 4.5 & $66 \pm 4$ & 0.13 \\
\hline 4119.008 & 28730.712 & ev & 3.5 & 4459.872 & od & 3.5 & $14.7 \pm 0.8$ & -0.53 \\
\hline 4119.883 & 26900.354 & ev & 3.5 & 2634.666 & od & 2.5 & $18.7 \pm 1.2$ & -0.42 \\
\hline 4119.953 & 28725.148 & ev & 4.5 & 4459.872 & od & 3.5 & $2.30 \pm 0.19$ & -1.23 \\
\hline 4120.827 & 26841.384 & ev & 4.5 & 2581.257 & od & 4.5 & $16.6 \pm 0.9$ & -0.37 \\
\hline 4121.266 & 29908.904 & ev & 4.5 & 5651.357 & od & 5.5 & $0.39 \pm 0.04$ & -2.00 \\
\hline 4121.591 & 29166.597 & ev & 4.5 & 4910.963 & od & 5.5 & $3.08 \pm 0.20$ & -1.11 \\
\hline 4123.220 & 30065.164 & ev & 3.5 & 5819.113 & od & 4.5 & $15.8 \pm 1.3$ & -0.49 \\
\hline 4123.869 & 31155.623 & ev & 6.5 & 6913.392 & od & 6.5 & $68 \pm 4$ & 0.39 \\
\hline 4124.787 & 29750.547 & od & 5.5 & 5513.709 & ev & 5.5 & $25.7 \pm 1.4$ & -0.10 \\
\hline
\end{tabular}


Table 2

(Continued)

\begin{tabular}{|c|c|c|c|c|c|c|c|c|}
\hline$\lambda_{\text {air }}(\AA)$ & $E_{\text {upper }}\left(\mathrm{cm}^{-1}\right)$ & Parity & $J_{\text {upp }}$ & $E_{\text {lower }}\left(\mathrm{cm}^{-1}\right)$ & Parity & $J_{\text {low }}$ & $A$-value $\left(10^{6} \mathrm{~s}^{-1}\right)$ & $\log (g f)$ \\
\hline 4125.416 & 29908.904 & ev & 4.5 & 5675.763 & od & 4.5 & $1.20 \pm 0.11$ & -1.51 \\
\hline 4125.773 & 27934.638 & ev & 4.5 & 3703.594 & od & 3.5 & $2.67 \pm 0.18$ & -1.17 \\
\hline 4126.652 & 28685.758 & ev & 2.5 & 4459.872 & od & 3.5 & $7.4 \pm 0.6$ & -0.95 \\
\hline 4127.099 & 30166.057 & ev & 3.5 & 5942.798 & od & 3.5 & $3.55 \pm 0.29$ & -1.14 \\
\hline 4127.364 & 29735.413 & od & 4.5 & 5513.709 & ev & 5.5 & $79 \pm 4$ & 0.31 \\
\hline 4127.748 & 28730.712 & ev & 3.5 & 4511.257 & od & 2.5 & $22.4 \pm 1.2$ & -0.34 \\
\hline 4128.061 & 27811.496 & ev & 3.5 & 3593.882 & od & 4.5 & $11.8 \pm 1.1$ & -0.62 \\
\hline 4128.360 & 31738.484 & ev & 5.5 & 7522.622 & od & 5.5 & $16.9 \pm 1.7$ & -0.28 \\
\hline 4129.174 & 30180.096 & ev & 6.5 & 5969.007 & od & 5.5 & $4.6 \pm 0.4$ & -0.78 \\
\hline 4130.705 & 28725.148 & ev & 4.5 & 4523.033 & od & 4.5 & $24.9 \pm 1.3$ & -0.20 \\
\hline 4132.315 & 29908.904 & ev & 4.5 & 5716.216 & od & 3.5 & $8.1 \pm 0.7$ & -0.68 \\
\hline 4132.626 & 30829.124 & ev & 3.5 & 6638.258 & od & 4.5 & $8.9 \pm 1.0$ & -0.74 \\
\hline 4135.424 & 28685.758 & ev & 2.5 & 4511.257 & od & 2.5 & $21.2 \pm 1.4$ & -0.49 \\
\hline 4136.750 & 29449.778 & ev & 1.5 & 5283.029 & od & 0.5 & $5.4 \pm 0.5$ & -1.25 \\
\hline 4136.895 & 30134.910 & ev & 5.5 & 5969.007 & od & 5.5 & $5.9 \pm 0.4$ & -0.74 \\
\hline 4137.466 & 29281.374 & ev & 2.5 & 5118.806 & od & 2.5 & $21.1 \pm 1.3$ & -0.49 \\
\hline 4137.645 & 28327.071 & od & 5.5 & 4165.550 & ev & 4.5 & $82 \pm 4$ & 0.40 \\
\hline 4140.747 & 28345.313 & ev & 0.5 & 4201.893 & od & 1.5 & $12.5 \pm 0.7$ & -1.19 \\
\hline 4142.034 & 28337.814 & ev & 2.5 & 4201.893 & od & 1.5 & $1.82 \pm 0.17$ & -1.55 \\
\hline 4142.397 & 29750.547 & od & 5.5 & 5616.739 & ev & 4.5 & $54.3 \pm 2.8$ & 0.22 \\
\hline 4142.825 & 29807.078 & ev & 3.5 & 5675.763 & od & 4.5 & $15.6 \pm 0.9$ & -0.49 \\
\hline 4144.362 & 30065.164 & ev & 3.5 & 5942.798 & od & 3.5 & $3.40 \pm 0.23$ & -1.15 \\
\hline 4144.492 & 27975.619 & od & 4.5 & 3854.012 & ev & 3.5 & $17.9 \pm 1.0$ & -0.34 \\
\hline 4144.847 & 30637.157 & ev & 2.5 & 6517.619 & od & 2.5 & $7.6 \pm 0.8$ & -0.93 \\
\hline 4144.996 & 29735.413 & od & 4.5 & 5616.739 & ev & 4.5 & $49.1 \pm 2.6$ & 0.10 \\
\hline 4145.486 & 30637.157 & ev & 2.5 & 6521.332 & od & 1.5 & $1.56 \pm 0.14$ & -1.62 \\
\hline 4146.232 & 28634.516 & ev & 5.5 & 4523.033 & od & 4.5 & $24.6 \pm 1.4$ & -0.12 \\
\hline 4148.162 & 30065.164 & ev & 3.5 & 5964.896 & od & 3.5 & $8.4 \pm 0.6$ & -0.76 \\
\hline 4149.143 & 32372.621 & od & 4.5 & 8278.054 & ev & 5.5 & $3.72 \pm 0.27$ & -1.02 \\
\hline 4149.781 & 29807.078 & ev & 3.5 & 5716.216 & od & 3.5 & $8.2 \pm 0.6$ & -0.77 \\
\hline 4149.966 & 29908.904 & ev & 4.5 & 5819.113 & od & 4.5 & $34.0 \pm 2.1$ & -0.06 \\
\hline 4150.404 & 30637.157 & ev & 2.5 & 6549.908 & od & 2.5 & $0.97 \pm 0.10$ & -1.82 \\
\hline 4151.970 & 29591.873 & od & 6.5 & 5513.709 & ev & 5.5 & $75 \pm 4$ & 0.43 \\
\hline 4153.126 & 25945.396 & ev & 3.5 & 1873.934 & od & 3.5 & $7.6 \pm 0.4$ & -0.80 \\
\hline 4153.406 & 29994.041 & ev & 2.5 & 5924.204 & od & 1.5 & $2.03 \pm 0.21$ & -1.50 \\
\hline 4160.106 & 28297.473 & ev & 3.5 & 4266.397 & od & 3.5 & $3.6 \pm 0.3$ & -1.12 \\
\hline 4160.440 & 29994.041 & ev & 2.5 & 5964.896 & od & 3.5 & $0.59 \pm 0.06$ & -2.04 \\
\hline 4162.872 & 28337.814 & ev & 2.5 & 4322.708 & od & 2.5 & $1.54 \pm 0.15$ & -1.62 \\
\hline 4165.599 & 31340.393 & od & 6.5 & 7341.007 & ev & 5.5 & $90 \pm 5$ & 0.52 \\
\hline 4165.850 & 32802.165 & ev & 5.5 & 8804.224 & od & 4.5 & $6.6 \pm 0.7$ & -0.68 \\
\hline 4166.649 & 28730.712 & ev & 3.5 & 4737.373 & od & 2.5 & $10.3 \pm 0.6$ & -0.67 \\
\hline 4167.582 & 29807.078 & ev & 3.5 & 5819.113 & od & 4.5 & $2.39 \pm 0.14$ & -1.30 \\
\hline 4169.766 & 29794.517 & ev & 3.5 & 5819.113 & od & 4.5 & $13.6 \pm 1.0$ & -0.55 \\
\hline 4169.877 & 28297.473 & ev & 3.5 & 4322.708 & od & 2.5 & $21.2 \pm 1.1$ & -0.35 \\
\hline 4171.384 & 29908.904 & ev & 4.5 & 5942.798 & od & 3.5 & $3.2 \pm 0.4$ & -1.08 \\
\hline 4172.152 & 26841.384 & ev & 4.5 & 2879.695 & od & 5.5 & $3.26 \pm 0.19$ & -1.07 \\
\hline 4174.470 & 28685.758 & ev & 2.5 & 4737.373 & od & 2.5 & $4.9 \pm 0.3$ & -1.11 \\
\hline 4175.233 & 29908.904 & ev & 4.5 & 5964.896 & od & 3.5 & $4.2 \pm 0.3$ & -0.95 \\
\hline 4176.076 & 27934.638 & ev & 4.5 & 3995.460 & od & 3.5 & $3.84 \pm 0.26$ & -1.00 \\
\hline 4179.075 & 31155.623 & ev & 6.5 & 7233.627 & od & 5.5 & $3.11 \pm 0.22$ & -0.94 \\
\hline 4185.331 & 27249.669 & ev & 2.5 & 3363.427 & od & 2.5 & $16.4 \pm 0.9$ & -0.59 \\
\hline 4187.322 & 28334.756 & ev & 4.5 & 4459.872 & od & 3.5 & $14.2 \pm 0.8$ & -0.43 \\
\hline 4189.183 & 29807.078 & ev & 3.5 & 5942.798 & od & 3.5 & $3.45 \pm 0.22$ & -1.14 \\
\hline 4189.638 & 31155.623 & ev & 6.5 & 7293.938 & od & 6.5 & $3.41 \pm 0.18$ & -0.90 \\
\hline 4192.754 & 29281.374 & ev & 2.5 & 5437.422 & od & 3.5 & $4.2 \pm 0.3$ & -1.18 \\
\hline 4193.065 & 29807.078 & ev & 3.5 & 5964.896 & od & 3.5 & $6.6 \pm 0.6$ & -0.85 \\
\hline 4193.283 & 32372.621 & od & 4.5 & 8531.678 & ev & 3.5 & $41.7 \pm 2.4$ & 0.04 \\
\hline 4193.871 & 28297.473 & ev & 3.5 & 4459.872 & od & 3.5 & $15.6 \pm 0.8$ & -0.48 \\
\hline 4195.276 & 29794.517 & ev & 3.5 & 5964.896 & od & 3.5 & $2.80 \pm 0.23$ & -1.23 \\
\hline 4195.815 & 28337.814 & ev & 2.5 & 4511.257 & od & 2.5 & $6.8 \pm 0.4$ & -0.97 \\
\hline 4196.332 & 27187.047 & ev & 3.5 & 3363.427 & od & 2.5 & $21.6 \pm 1.2$ & -0.34 \\
\hline 4197.510 & 27812.398 & ev & 2.5 & 3995.460 & od & 3.5 & $5.8 \pm 0.4$ & -1.03 \\
\hline 4197.669 & 27811.496 & ev & 3.5 & 3995.460 & od & 3.5 & $9.1 \pm 0.6$ & -0.72 \\
\hline 4198.429 & 28334.756 & ev & 4.5 & 4523.033 & od & 4.5 & $8.2 \pm 0.5$ & -0.66 \\
\hline 4198.721 & 27975.619 & od & 4.5 & 4165.550 & ev & 4.5 & $18.0 \pm 1.0$ & -0.32 \\
\hline 4202.712 & 29438.817 & ev & 5.5 & 5651.357 & od & 5.5 & $0.57 \pm 0.09$ & -1.74 \\
\hline 4202.931 & 28297.473 & ev & 3.5 & 4511.257 & od & 2.5 & $22.0 \pm 1.2$ & -0.33 \\
\hline
\end{tabular}


Table 2

(Continued)

\begin{tabular}{|c|c|c|c|c|c|c|c|c|}
\hline$\lambda_{\text {air }}(\AA)$ & $E_{\text {upper }}\left(\mathrm{cm}^{-1}\right)$ & Parity & $J_{\text {upp }}$ & $E_{\text {lower }}\left(\mathrm{cm}^{-1}\right)$ & Parity & $J_{\text {low }}$ & $A$-value $\left(10^{6} \mathrm{~s}^{-1}\right)$ & $\log (g f)$ \\
\hline 4202.958 & 27379.949 & ev & 5.5 & 3593.882 & od & 4.5 & $10.8 \pm 0.6$ & -0.46 \\
\hline 4204.717 & 30166.057 & ev & 3.5 & 6389.942 & od & 4.5 & $2.47 \pm 0.20$ & -1.28 \\
\hline 4205.013 & 28297.473 & ev & 3.5 & 4523.033 & od & 4.5 & $0.32 \pm 0.05$ & -2.17 \\
\hline 4207.028 & 29438.817 & ev & 5.5 & 5675.763 & od & 4.5 & $0.107 \pm 0.016$ & -2.47 \\
\hline 4209.406 & 29263.338 & od & 5.5 & 5513.709 & ev & 5.5 & $7.8 \pm 0.5$ & -0.61 \\
\hline 4210.233 & 30134.910 & ev & 5.5 & 6389.942 & od & 4.5 & $1.15 \pm 0.06$ & -1.44 \\
\hline 4213.035 & 29166.597 & ev & 4.5 & 5437.422 & od & 3.5 & $4.30 \pm 0.24$ & -0.94 \\
\hline 4214.033 & 28634.516 & ev & 5.5 & 4910.963 & od & 5.5 & $11.1 \pm 0.8$ & -0.45 \\
\hline 4222.597 & 24663.053 & ev & 4.5 & 987.611 & od & 4.5 & $26.7 \pm 1.4$ & -0.15 \\
\hline 4223.881 & 27934.638 & ev & 4.5 & 4266.397 & od & 3.5 & $4.5 \pm 0.3$ & -0.92 \\
\hline 4227.747 & 29263.338 & od & 5.5 & 5616.739 & ev & 4.5 & $25.0 \pm 1.3$ & -0.10 \\
\hline 4228.295 & 30702.610 & ev & 4.5 & 7059.072 & od & 4.5 & $5.0 \pm 0.3$ & -0.87 \\
\hline 4230.119 & 27835.233 & ev & 1.5 & 4201.893 & od & 1.5 & $3.90 \pm 0.24$ & -1.38 \\
\hline 4230.180 & 31155.623 & ev & 6.5 & 7522.622 & od & 5.5 & $0.96 \pm 0.15$ & -1.44 \\
\hline 4231.327 & 30829.124 & ev & 3.5 & 7202.529 & od & 2.5 & $0.51 \pm 0.04$ & -1.96 \\
\hline 4232.561 & 29438.817 & ev & 5.5 & 5819.113 & od & 4.5 & $5.3 \pm 0.3$ & -0.77 \\
\hline 4233.959 & 28730.712 & ev & 3.5 & 5118.806 & od & 2.5 & $1.68 \pm 0.15$ & -1.44 \\
\hline 4234.210 & 27812.398 & ev & 2.5 & 4201.893 & od & 1.5 & $14.3 \pm 0.9$ & -0.64 \\
\hline 4234.728 & 30245.878 & ev & 4.5 & 6638.258 & od & 4.5 & $4.8 \pm 0.3$ & -0.89 \\
\hline 4236.016 & 28337.814 & ev & 2.5 & 4737.373 & od & 2.5 & $15.0 \pm 0.8$ & -0.62 \\
\hline 4236.354 & 32372.621 & od & 4.5 & 8774.064 & ev & 4.5 & $10.3 \pm 0.9$ & -0.56 \\
\hline 4238.553 & 27379.949 & ev & 5.5 & 3793.634 & od & 6.5 & $0.59 \pm 0.07$ & -1.72 \\
\hline 4239.909 & 27432.782 & od & 4.5 & 3854.012 & ev & 3.5 & $36.8 \pm 1.9$ & 0.00 \\
\hline 4242.359 & 29281.374 & ev & 2.5 & 5716.216 & od & 3.5 & $0.60 \pm 0.07$ & -2.01 \\
\hline 4242.720 & 25945.396 & ev & 3.5 & 2382.246 & od & 4.5 & $11.9 \pm 0.6$ & -0.59 \\
\hline 4245.973 & 27811.496 & ev & 3.5 & 4266.397 & od & 3.5 & $15.4 \pm 0.9$ & -0.48 \\
\hline 4246.713 & 25681.488 & $\mathrm{ev}$ & 1.5 & 2140.492 & od & 0.5 & $25.6 \pm 1.3$ & -0.56 \\
\hline 4247.447 & 26900.354 & ev & 3.5 & 3363.427 & od & 2.5 & $2.07 \pm 0.18$ & -1.35 \\
\hline 4248.671 & 29043.854 & od & 6.5 & 5513.709 & ev & 5.5 & $40.3 \pm 2.1$ & 0.18 \\
\hline 4250.692 & 29908.904 & ev & 4.5 & 6389.942 & od & 4.5 & $1.23 \pm 0.09$ & -1.48 \\
\hline 4251.855 & 27835.233 & ev & 1.5 & 4322.708 & od & 2.5 & $7.3 \pm 0.6$ & -1.10 \\
\hline 4253.362 & 27249.669 & ev & 2.5 & 3745.475 & od & 1.5 & $17.4 \pm 0.9$ & -0.55 \\
\hline 4254.000 & 28345.313 & ev & 0.5 & 4844.644 & od & 1.5 & $1.38 \pm 0.16$ & -2.13 \\
\hline 4254.728 & 30134.910 & ev & 5.5 & 6638.258 & od & 4.5 & $0.88 \pm 0.08$ & -1.54 \\
\hline 4255.358 & 28337.814 & ev & 2.5 & 4844.644 & od & 1.5 & $3.31 \pm 0.23$ & -1.27 \\
\hline 4255.782 & 29166.597 & ev & 4.5 & 5675.763 & od & 4.5 & $27.0 \pm 1.4$ & -0.13 \\
\hline 4255.989 & 27812.398 & ev & 2.5 & 4322.708 & od & 2.5 & $6.6 \pm 0.6$ & -0.97 \\
\hline 4256.152 & 27811.496 & ev & 3.5 & 4322.708 & od & 2.5 & $8.3 \pm 0.6$ & -0.74 \\
\hline 4256.702 & 25359.686 & $\mathrm{ev}$ & 2.5 & 1873.934 & od & 3.5 & $0.138 \pm 0.013$ & -2.65 \\
\hline 4257.119 & 27187.047 & ev & 3.5 & 3703.594 & od & 3.5 & $3.33 \pm 0.24$ & -1.14 \\
\hline 4258.394 & 29994.041 & ev & 2.5 & 6517.619 & od & 2.5 & $3.8 \pm 0.3$ & -1.20 \\
\hline 4259.068 & 29994.041 & ev & 2.5 & 6521.332 & od & 1.5 & $2.60 \pm 0.20$ & -1.37 \\
\hline 4259.594 & 29438.817 & ev & 5.5 & 5969.007 & od & 5.5 & $0.73 \pm 0.08$ & -1.62 \\
\hline 4259.744 & 30702.610 & ev & 4.5 & 7233.627 & od & 5.5 & $7.5 \pm 0.5$ & -0.69 \\
\hline 4264.368 & 30702.610 & ev & 4.5 & 7259.075 & od & 3.5 & $10.2 \pm 0.7$ & -0.56 \\
\hline 4269.176 & 29807.078 & ev & 3.5 & 6389.942 & od & 4.5 & $1.78 \pm 0.17$ & -1.41 \\
\hline 4270.184 & 27934.638 & ev & 4.5 & 4523.033 & od & 4.5 & $19.6 \pm 1.2$ & -0.27 \\
\hline 4278.859 & 25945.396 & ev & 3.5 & 2581.257 & od & 4.5 & $6.3 \pm 0.3$ & -0.86 \\
\hline 4279.941 & 30637.157 & ev & 2.5 & 7278.922 & od & 1.5 & $0.57 \pm 0.07$ & -2.03 \\
\hline 4280.136 & 29281.374 & ev & 2.5 & 5924.204 & od & 1.5 & $12.6 \pm 0.7$ & -0.68 \\
\hline 4280.987 & 27812.398 & ev & 2.5 & 4459.872 & od & 3.5 & $6.9 \pm 0.4$ & -0.95 \\
\hline 4281.153 & 27811.496 & ev & 3.5 & 4459.872 & od & 3.5 & $4.8 \pm 0.3$ & -0.98 \\
\hline 4283.546 & 29281.374 & ev & 2.5 & 5942.798 & od & 3.5 & $3.30 \pm 0.23$ & -1.26 \\
\hline 4287.144 & 32372.621 & od & 4.5 & 9053.629 & ev & 3.5 & $1.72 \pm 0.15$ & -1.32 \\
\hline 4287.606 & 29281.374 & ev & 2.5 & 5964.896 & od & 3.5 & $1.49 \pm 0.13$ & -1.61 \\
\hline 4288.663 & 25945.396 & ev & 3.5 & 2634.666 & od & 2.5 & $5.25 \pm 0.28$ & -0.94 \\
\hline 4289.447 & 26900.354 & ev & 3.5 & 3593.882 & od & 4.5 & $8.0 \pm 0.6$ & -0.75 \\
\hline 4289.932 & 25945.396 & ev & 3.5 & 2641.559 & od & 3.5 & $58.4 \pm 3.0$ & 0.11 \\
\hline 4290.428 & 27812.398 & ev & 2.5 & 4511.257 & od & 2.5 & $1.63 \pm 0.15$ & -1.57 \\
\hline 4290.594 & 27811.496 & ev & 3.5 & 4511.257 & od & 2.5 & $1.51 \pm 0.10$ & -1.48 \\
\hline 4291.874 & 28730.712 & ev & 3.5 & 5437.422 & od & 3.5 & $0.238 \pm 0.028$ & -2.28 \\
\hline 4292.580 & 29807.078 & ev & 3.5 & 6517.619 & od & 2.5 & $8.3 \pm 0.7$ & -0.74 \\
\hline 4292.764 & 27811.496 & ev & 3.5 & 4523.033 & od & 4.5 & $6.3 \pm 0.4$ & -0.85 \\
\hline 4292.900 & 28725.148 & ev & 4.5 & 5437.422 & od & 3.5 & $1.61 \pm 0.13$ & -1.35 \\
\hline 4294.897 & 29794.517 & ev & 3.5 & 6517.619 & od & 2.5 & $1.80 \pm 0.13$ & -1.40 \\
\hline 4296.051 & 29908.904 & ev & 4.5 & 6638.258 & od & 4.5 & $5.1 \pm 0.5$ & -0.85 \\
\hline
\end{tabular}


Table 2

(Continued)

\begin{tabular}{|c|c|c|c|c|c|c|c|c|}
\hline$\lambda_{\text {air }}(\AA)$ & $E_{\text {upper }}\left(\mathrm{cm}^{-1}\right)$ & Parity & $J_{\text {upp }}$ & $E_{\text {lower }}\left(\mathrm{cm}^{-1}\right)$ & Parity & $J_{\text {low }}$ & $A$-value $\left(10^{6} \mathrm{~s}^{-1}\right)$ & $\log (g f)$ \\
\hline 4296.681 & 27432.782 & od & 4.5 & 4165.550 & ev & 4.5 & $53.2 \pm 2.7$ & 0.17 \\
\hline 4296.779 & 30180.096 & ev & 6.5 & 6913.392 & od & 6.5 & $23.5 \pm 1.3$ & -0.04 \\
\hline 4299.087 & 27249.669 & ev & 2.5 & 3995.460 & od & 3.5 & $4.71 \pm 0.28$ & -1.11 \\
\hline 4299.357 & 24663.053 & ev & 4.5 & 1410.304 & od & 4.5 & $9.9 \pm 0.5$ & -0.56 \\
\hline 4300.328 & 26841.384 & ev & 4.5 & 3593.882 & od & 4.5 & $22.1 \pm 1.1$ & -0.21 \\
\hline 4300.863 & 29794.517 & ev & 3.5 & 6549.908 & od & 2.5 & $3.80 \pm 0.26$ & -1.07 \\
\hline 4304.717 & 29166.597 & ev & 4.5 & 5942.798 & od & 3.5 & $7.4 \pm 0.4$ & -0.69 \\
\hline 4305.140 & 30134.910 & ev & 5.5 & 6913.392 & od & 6.5 & $20.3 \pm 1.2$ & -0.17 \\
\hline 4305.605 & 28337.814 & ev & 2.5 & 5118.806 & od & 2.5 & $2.93 \pm 0.26$ & -1.31 \\
\hline 4306.726 & 27378.515 & od & 5.5 & 4165.550 & ev & 4.5 & $24.2 \pm 1.3$ & -0.09 \\
\hline 4309.580 & 29166.597 & ev & 4.5 & 5969.007 & od & 5.5 & $4.7 \pm 0.3$ & -0.88 \\
\hline 4309.735 & 26900.354 & ev & 3.5 & 3703.594 & od & 3.5 & $15.5 \pm 1.0$ & -0.46 \\
\hline 4310.696 & 27187.047 & ev & 3.5 & 3995.460 & od & 3.5 & $5.1 \pm 0.4$ & -0.94 \\
\hline 4311.585 & 30245.878 & ev & 4.5 & 7059.072 & od & 4.5 & $7.5 \pm 0.5$ & -0.68 \\
\hline 4312.853 & 30702.610 & ev & 4.5 & 7522.622 & od & 5.5 & $0.89 \pm 0.09$ & -1.60 \\
\hline 4313.099 & 28297.473 & ev & 3.5 & 5118.806 & od & 2.5 & $2.26 \pm 0.14$ & -1.30 \\
\hline 4313.592 & 27379.949 & ev & 5.5 & 4203.934 & od & 6.5 & $0.77 \pm 0.06$ & -1.59 \\
\hline 4314.932 & 29807.078 & ev & 3.5 & 6638.258 & od & 4.5 & $3.04 \pm 0.22$ & -1.17 \\
\hline 4317.273 & 29794.517 & ev & 3.5 & 6638.258 & od & 4.5 & $1.29 \pm 0.23$ & -1.54 \\
\hline 4320.719 & 26841.384 & ev & 4.5 & 3703.594 & od & 3.5 & $14.5 \pm 0.8$ & -0.39 \\
\hline 4324.785 & 30829.124 & ev & 3.5 & 7713.089 & od & 4.5 & $16.7 \pm 1.3$ & -0.43 \\
\hline 4326.479 & 30166.057 & ev & 3.5 & 7059.072 & od & 4.5 & $0.76 \pm 0.08$ & -1.77 \\
\hline 4330.441 & 25681.488 & ev & 1.5 & 2595.644 & od & 1.5 & $13.0 \pm 0.7$ & -0.83 \\
\hline 4331.757 & 32802.165 & ev & 5.5 & 9723.335 & od & 4.5 & $13.1 \pm 0.9$ & -0.35 \\
\hline 4332.319 & 30134.910 & ev & 5.5 & 7059.072 & od & 4.5 & $0.56 \pm 0.06$ & -1.72 \\
\hline 4332.472 & 27812.398 & ev & 2.5 & 4737.373 & od & 2.5 & $1.29 \pm 0.11$ & -1.66 \\
\hline 4332.641 & 27811.496 & ev & 3.5 & 4737.373 & od & 2.5 & $2.54 \pm 0.21$ & -1.24 \\
\hline 4332.703 & 28725.148 & ev & 4.5 & 5651.357 & od & 5.5 & $9.2 \pm 0.5$ & -0.59 \\
\hline 4337.291 & 28725.148 & ev & 4.5 & 5675.763 & od & 4.5 & $1.03 \pm 0.07$ & -1.54 \\
\hline 4337.387 & 29438.817 & ev & 5.5 & 6389.942 & od & 4.5 & $1.19 \pm 0.11$ & -1.40 \\
\hline 4337.594 & 27249.669 & ev & 2.5 & 4201.893 & od & 1.5 & $1.87 \pm 0.20$ & -1.50 \\
\hline 4337.773 & 25681.488 & ev & 1.5 & 2634.666 & od & 2.5 & $43.5 \pm 2.2$ & -0.31 \\
\hline 4342.135 & 27934.638 & ev & 4.5 & 4910.963 & od & 5.5 & $1.74 \pm 0.19$ & -1.31 \\
\hline 4343.866 & 28730.712 & ev & 3.5 & 5716.216 & od & 3.5 & $2.42 \pm 0.19$ & -1.26 \\
\hline 4344.917 & 28725.148 & ev & 4.5 & 5716.216 & od & 3.5 & $1.18 \pm 0.13$ & -1.48 \\
\hline 4345.453 & 30065.164 & ev & 3.5 & 7059.072 & od & 4.5 & $2.70 \pm 0.22$ & -1.21 \\
\hline 4348.585 & 30702.610 & ev & 4.5 & 7713.089 & od & 4.5 & $1.25 \pm 0.10$ & -1.45 \\
\hline 4349.768 & 27249.669 & ev & 2.5 & 4266.397 & od & 3.5 & $11.1 \pm 0.6$ & -0.73 \\
\hline 4349.789 & 28634.516 & ev & 5.5 & 5651.357 & od & 5.5 & $14.0 \pm 0.9$ & -0.32 \\
\hline 4352.707 & 27812.398 & ev & 2.5 & 4844.644 & od & 1.5 & $32.9 \pm 1.9$ & -0.25 \\
\hline 4355.923 & 30829.124 & ev & 3.5 & 7878.328 & od & 3.5 & $0.60 \pm 0.07$ & -1.86 \\
\hline 4356.744 & 30180.096 & ev & 6.5 & 7233.627 & od & 5.5 & $1.77 \pm 0.18$ & -1.15 \\
\hline 4359.064 & 31738.484 & ev & 5.5 & 8804.224 & od & 4.5 & $5.5 \pm 0.9$ & -0.72 \\
\hline 4360.169 & 29449.778 & ev & 1.5 & 6521.332 & od & 1.5 & $9.5 \pm 0.7$ & -0.97 \\
\hline 4361.652 & 27187.047 & ev & 3.5 & 4266.397 & od & 3.5 & $4.9 \pm 0.3$ & -0.95 \\
\hline 4364.653 & 26900.354 & ev & 3.5 & 3995.460 & od & 3.5 & $29.5 \pm 1.9$ & -0.17 \\
\hline 4365.341 & 30134.910 & ev & 5.5 & 7233.627 & od & 5.5 & $0.62 \pm 0.10$ & -1.67 \\
\hline 4365.511 & 28337.814 & ev & 2.5 & 5437.422 & od & 3.5 & $2.56 \pm 0.19$ & -1.36 \\
\hline 4366.094 & 28334.756 & ev & 4.5 & 5437.422 & od & 3.5 & $0.229 \pm 0.028$ & -2.18 \\
\hline 4368.226 & 30180.096 & ev & 6.5 & 7293.938 & od & 6.5 & $3.40 \pm 0.23$ & -0.87 \\
\hline 4369.235 & 29892.677 & od & 3.5 & 7011.804 & ev & 4.5 & $5.6 \pm 0.4$ & -0.89 \\
\hline 4372.394 & 27187.047 & ev & 3.5 & 4322.708 & od & 2.5 & $2.82 \pm 0.16$ & -1.19 \\
\hline 4373.215 & 28297.473 & ev & 3.5 & 5437.422 & od & 3.5 & $1.78 \pm 0.14$ & -1.39 \\
\hline 4373.814 & 27379.949 & ev & 5.5 & 4523.033 & od & 4.5 & $9.7 \pm 0.6$ & -0.48 \\
\hline 4375.170 & 29908.904 & ev & 4.5 & 7059.072 & od & 4.5 & $2.89 \pm 0.21$ & -1.08 \\
\hline 4375.919 & 26841.384 & ev & 4.5 & 3995.460 & od & 3.5 & $14.9 \pm 0.8$ & -0.37 \\
\hline 4376.868 & 30134.910 & ev & 5.5 & 7293.938 & od & 6.5 & $0.74 \pm 0.06$ & -1.59 \\
\hline 4380.053 & 27835.233 & ev & 1.5 & 5010.870 & od & 2.5 & $8.6 \pm 0.8$ & -1.01 \\
\hline 4381.080 & 30637.157 & ev & 2.5 & 7818.147 & od & 1.5 & $2.63 \pm 0.23$ & -1.34 \\
\hline 4381.773 & 28634.516 & ev & 5.5 & 5819.113 & od & 4.5 & $4.6 \pm 0.3$ & -0.80 \\
\hline 4382.165 & 28327.071 & od & 5.5 & 5513.709 & ev & 5.5 & $39.2 \pm 2.2$ & 0.13 \\
\hline 4384.439 & 27812.398 & ev & 2.5 & 5010.870 & od & 2.5 & $0.66 \pm 0.05$ & -1.94 \\
\hline 4386.366 & 29994.041 & ev & 2.5 & 7202.529 & od & 2.5 & $4.8 \pm 0.3$ & -1.08 \\
\hline 4386.696 & 27249.669 & ev & 2.5 & 4459.872 & od & 3.5 & $14.6 \pm 0.8$ & -0.60 \\
\hline 4386.827 & 24663.053 & ev & 4.5 & 1873.934 & od & 3.5 & $14.3 \pm 0.7$ & -0.38 \\
\hline
\end{tabular}


Table 2

(Continued)

\begin{tabular}{|c|c|c|c|c|c|c|c|c|}
\hline$\lambda_{\text {air }}(\AA)$ & $E_{\text {upper }}\left(\mathrm{cm}^{-1}\right)$ & Parity & $J_{\text {upp }}$ & $E_{\text {lower }}\left(\mathrm{cm}^{-1}\right)$ & Parity & $J_{\text {low }}$ & $A$-value $\left(10^{6} \mathrm{~s}^{-1}\right)$ & $\log (g f)$ \\
\hline 4387.059 & 28730.712 & ev & 3.5 & 5942.798 & od & 3.5 & $4.4 \pm 0.4$ & -0.99 \\
\hline 4388.005 & 29750.547 & od & 5.5 & 6967.547 & ev & 6.5 & $11.8 \pm 0.6$ & -0.39 \\
\hline 4390.760 & 32492.038 & ev & 5.5 & 9723.335 & od & 4.5 & $0.42 \pm 0.05$ & -1.84 \\
\hline 4391.317 & 28730.712 & ev & 3.5 & 5964.896 & od & 3.5 & $1.70 \pm 0.14$ & -1.41 \\
\hline 4391.659 & 25359.686 & ev & 2.5 & 2595.644 & od & 1.5 & $52.9 \pm 2.7$ & -0.04 \\
\hline 4393.184 & 28725.148 & ev & 4.5 & 5969.007 & od & 5.5 & $9.3 \pm 0.6$ & -0.57 \\
\hline 4396.609 & 27249.669 & ev & 2.5 & 4511.257 & od & 2.5 & $2.33 \pm 0.16$ & -1.39 \\
\hline 4397.183 & 29794.517 & ev & 3.5 & 7059.072 & od & 4.5 & $0.70 \pm 0.07$ & -1.79 \\
\hline 4397.276 & 29994.041 & ev & 2.5 & 7259.075 & od & 3.5 & $2.87 \pm 0.21$ & -1.30 \\
\hline 4398.783 & 27187.047 & ev & 3.5 & 4459.872 & od & 3.5 & $7.5 \pm 0.5$ & -0.76 \\
\hline 4399.200 & 25359.686 & ev & 2.5 & 2634.666 & od & 2.5 & $21.1 \pm 1.1$ & -0.44 \\
\hline 4399.474 & 29735.413 & od & 4.5 & 7011.804 & ev & 4.5 & $0.94 \pm 0.10$ & -1.56 \\
\hline 4399.542 & 30245.878 & ev & 4.5 & 7522.622 & od & 5.5 & $1.72 \pm 0.13$ & -1.30 \\
\hline 4400.535 & 25359.686 & ev & 2.5 & 2641.559 & od & 3.5 & $3.18 \pm 0.22$ & -1.26 \\
\hline 4400.865 & 27835.233 & ev & 1.5 & 5118.806 & od & 2.5 & $9.6 \pm 0.7$ & -0.95 \\
\hline 4405.293 & 27812.398 & ev & 2.5 & 5118.806 & od & 2.5 & $1.96 \pm 0.19$ & -1.47 \\
\hline 4405.468 & 27811.496 & ev & 3.5 & 5118.806 & od & 2.5 & $3.9 \pm 0.4$ & -1.04 \\
\hline 4407.272 & 28334.756 & ev & 4.5 & 5651.357 & od & 5.5 & $6.6 \pm 0.4$ & -0.71 \\
\hline 4412.020 & 28334.756 & ev & 4.5 & 5675.763 & od & 4.5 & $4.9 \pm 0.3$ & -0.84 \\
\hline 4416.900 & 26900.354 & ev & 3.5 & 4266.397 & od & 3.5 & $7.4 \pm 0.5$ & -0.76 \\
\hline 4418.780 & 29591.873 & od & 6.5 & 6967.547 & ev & 6.5 & $45.1 \pm 2.5$ & 0.27 \\
\hline 4427.069 & 25945.396 & ev & 3.5 & 3363.427 & od & 2.5 & $9.9 \pm 0.6$ & -0.63 \\
\hline 4427.208 & 28297.473 & ev & 3.5 & 5716.216 & od & 3.5 & $0.63 \pm 0.10$ & -1.83 \\
\hline 4427.916 & 26900.354 & ev & 3.5 & 4322.708 & od & 2.5 & $16.7 \pm 1.1$ & -0.41 \\
\hline 4428.438 & 26841.384 & ev & 4.5 & 4266.397 & od & 3.5 & $9.0 \pm 0.5$ & -0.58 \\
\hline 4432.912 & 27835.233 & ev & 1.5 & 5283.029 & od & 0.5 & $5.3 \pm 0.3$ & -1.20 \\
\hline 4433.738 & 29807.078 & $\mathrm{ev}$ & 3.5 & 7259.075 & od & 3.5 & $1.84 \pm 0.13$ & -1.36 \\
\hline 4436.209 & 29794.517 & ev & 3.5 & 7259.075 & od & 3.5 & $1.70 \pm 0.13$ & -1.40 \\
\hline 4440.110 & 28334.756 & ev & 4.5 & 5819.113 & od & 4.5 & $0.44 \pm 0.05$ & -1.89 \\
\hline 4443.747 & 27934.638 & ev & 4.5 & 5437.422 & od & 3.5 & $6.3 \pm 0.4$ & -0.73 \\
\hline 4449.330 & 27379.949 & ev & 5.5 & 4910.963 & od & 5.5 & $31.0 \pm 1.8$ & 0.04 \\
\hline 4449.633 & 30637.157 & ev & 2.5 & 8169.698 & od & 1.5 & $10.2 \pm 1.0$ & -0.74 \\
\hline 4450.732 & 27975.619 & od & 4.5 & 5513.709 & ev & 5.5 & $23.0 \pm 1.2$ & -0.17 \\
\hline 4450.854 & 30637.157 & ev & 2.5 & 8175.863 & od & 2.5 & $2.17 \pm 0.23$ & -1.41 \\
\hline 4452.504 & 30166.057 & ev & 3.5 & 7713.089 & od & 4.5 & $1.23 \pm 0.18$ & -1.53 \\
\hline 4453.158 & 27187.047 & ev & 3.5 & 4737.373 & od & 2.5 & $1.94 \pm 0.19$ & -1.34 \\
\hline 4454.982 & 26900.354 & ev & 3.5 & 4459.872 & od & 3.5 & $3.16 \pm 0.24$ & -1.12 \\
\hline 4457.768 & 30829.124 & ev & 3.5 & 8402.668 & od & 3.5 & $4.3 \pm 0.4$ & -0.99 \\
\hline 4458.831 & 28345.313 & ev & 0.5 & 5924.204 & od & 1.5 & $1.49 \pm 0.17$ & -2.05 \\
\hline 4459.077 & 30166.057 & ev & 3.5 & 7746.185 & od & 2.5 & $1.08 \pm 0.10$ & -1.59 \\
\hline 4460.207 & 26268.203 & od & 3.5 & 3854.012 & ev & 3.5 & $80 \pm 4$ & 0.28 \\
\hline 4461.133 & 29750.547 & od & 5.5 & 7341.007 & ev & 5.5 & $23.1 \pm 1.3$ & -0.08 \\
\hline 4462.032 & 27249.669 & ev & 2.5 & 4844.644 & od & 1.5 & $0.338 \pm 0.028$ & -2.22 \\
\hline 4465.437 & 29449.778 & ev & 1.5 & 7061.838 & od & 0.5 & $5.8 \pm 0.4$ & -1.16 \\
\hline 4466.720 & 26841.384 & ev & 4.5 & 4459.872 & od & 3.5 & $0.48 \pm 0.05$ & -1.84 \\
\hline 4467.073 & 29438.817 & ev & 5.5 & 7059.072 & od & 4.5 & $1.05 \pm 0.15$ & -1.42 \\
\hline 4468.025 & 27812.398 & ev & 2.5 & 5437.422 & od & 3.5 & $0.50 \pm 0.06$ & -2.04 \\
\hline 4469.508 & 30245.878 & ev & 4.5 & 7878.328 & od & 3.5 & $0.44 \pm 0.04$ & -1.88 \\
\hline 4471.241 & 27975.619 & od & 4.5 & 5616.739 & ev & 4.5 & $56.0 \pm 2.9$ & 0.23 \\
\hline 4472.082 & 28297.473 & ev & 3.5 & 5942.798 & od & 3.5 & $0.81 \pm 0.08$ & -1.71 \\
\hline 4472.602 & 30065.164 & ev & 3.5 & 7713.089 & od & 4.5 & $6.3 \pm 0.6$ & -0.82 \\
\hline 4472.715 & 25945.396 & ev & 3.5 & 3593.882 & od & 4.5 & $10.6 \pm 0.6$ & -0.60 \\
\hline 4479.235 & 30065.164 & ev & 3.5 & 7746.185 & od & 2.5 & $7.3 \pm 0.5$ & -0.75 \\
\hline 4479.361 & 26841.384 & ev & 4.5 & 4523.033 & od & 4.5 & $12.0 \pm 0.7$ & -0.44 \\
\hline 4479.419 & 25681.488 & ev & 1.5 & 3363.427 & od & 2.5 & $9.8 \pm 0.7$ & -0.93 \\
\hline 4483.059 & 30702.610 & ev & 4.5 & 8402.668 & od & 3.5 & $0.84 \pm 0.11$ & -1.60 \\
\hline 4483.893 & 29263.338 & od & 5.5 & 6967.547 & ev & 6.5 & $34.9 \pm 1.8$ & 0.10 \\
\hline 4485.515 & 30166.057 & ev & 3.5 & 7878.328 & od & 3.5 & $9.3 \pm 0.8$ & -0.65 \\
\hline 4486.909 & 24663.053 & ev & 4.5 & 2382.246 & od & 4.5 & $22.0 \pm 1.2$ & -0.18 \\
\hline 4492.947 & 29591.873 & od & 6.5 & 7341.007 & ev & 5.5 & $1.99 \pm 0.13$ & -1.07 \\
\hline 4493.554 & 29994.041 & ev & 2.5 & 7746.185 & od & 2.5 & $0.59 \pm 0.07$ & -1.97 \\
\hline 4494.217 & 28634.516 & ev & 5.5 & 6389.942 & od & 4.5 & $5.9 \pm 0.5$ & -0.67 \\
\hline 4495.385 & 27249.669 & ev & 2.5 & 5010.870 & od & 2.5 & $7.1 \pm 0.5$ & -0.89 \\
\hline 4496.256 & 30637.157 & ev & 2.5 & 8402.668 & od & 3.5 & $8.0 \pm 0.7$ & -0.84 \\
\hline 4499.507 & 27934.638 & ev & 4.5 & 5716.216 & od & 3.5 & $0.52 \pm 0.06$ & -1.80 \\
\hline 4508.079 & 27187.047 & ev & 3.5 & 5010.870 & od & 2.5 & $3.39 \pm 0.27$ & -1.08 \\
\hline
\end{tabular}


Table 2

(Continued)

\begin{tabular}{|c|c|c|c|c|c|c|c|c|}
\hline$\lambda_{\text {air }}(\AA)$ & $E_{\text {upper }}\left(\mathrm{cm}^{-1}\right)$ & Parity & $J_{\text {upp }}$ & $E_{\text {lower }}\left(\mathrm{cm}^{-1}\right)$ & Parity & $J_{\text {low }}$ & $A$-value $\left(10^{6} \mathrm{~s}^{-1}\right)$ & $\log (g f)$ \\
\hline 4508.721 & 25681.488 & ev & 1.5 & 3508.470 & od & 0.5 & $2.10 \pm 0.16$ & -1.59 \\
\hline 4509.255 & 29892.677 & od & 3.5 & 7722.285 & ev & 2.5 & $3.48 \pm 0.24$ & -1.07 \\
\hline 4510.763 & 26900.354 & ev & 3.5 & 4737.373 & od & 2.5 & $0.41 \pm 0.05$ & -2.00 \\
\hline 4520.404 & 29994.041 & ev & 2.5 & 7878.328 & od & 3.5 & $1.96 \pm 0.26$ & -1.44 \\
\hline 4523.075 & 26268.203 & od & 3.5 & 4165.550 & ev & 4.5 & $34.2 \pm 1.8$ & -0.08 \\
\hline 4524.849 & 29807.078 & ev & 3.5 & 7713.089 & od & 4.5 & $1.11 \pm 0.09$ & -1.56 \\
\hline 4527.348 & 24663.053 & ev & 4.5 & 2581.257 & od & 4.5 & $21.0 \pm 1.2$ & -0.19 \\
\hline 4527.423 & 29794.517 & ev & 3.5 & 7713.089 & od & 4.5 & $4.6 \pm 0.5$ & -0.95 \\
\hline 4527.953 & 29281.374 & ev & 2.5 & 7202.529 & od & 2.5 & $2.07 \pm 0.16$ & -1.42 \\
\hline 4528.473 & 29043.854 & od & 6.5 & 6967.547 & ev & 6.5 & $45.2 \pm 2.4$ & 0.29 \\
\hline 4534.219 & 29794.517 & ev & 3.5 & 7746.185 & od & 2.5 & $2.58 \pm 0.30$ & -1.20 \\
\hline 4537.874 & 29908.904 & ev & 4.5 & 7878.328 & od & 3.5 & $4.0 \pm 0.4$ & -0.90 \\
\hline 4539.745 & 24663.053 & ev & 4.5 & 2641.559 & od & 3.5 & $26.7 \pm 1.4$ & -0.08 \\
\hline 4544.953 & 25359.686 & ev & 2.5 & 3363.427 & od & 2.5 & $8.8 \pm 0.5$ & -0.79 \\
\hline 4545.867 & 27934.638 & ev & 4.5 & 5942.798 & od & 3.5 & $1.89 \pm 0.17$ & -1.23 \\
\hline 4551.291 & 27934.638 & ev & 4.5 & 5969.007 & od & 5.5 & $12.2 \pm 0.8$ & -0.42 \\
\hline 4554.545 & 25945.396 & ev & 3.5 & 3995.460 & od & 3.5 & $1.86 \pm 0.11$ & -1.33 \\
\hline 4555.608 & 28334.756 & ev & 4.5 & 6389.942 & od & 4.5 & $1.07 \pm 0.09$ & -1.48 \\
\hline 4558.068 & 29166.597 & ev & 4.5 & 7233.627 & od & 5.5 & $0.49 \pm 0.06$ & -1.82 \\
\hline 4558.598 & 26841.384 & ev & 4.5 & 4910.963 & od & 5.5 & $3.38 \pm 0.21$ & -0.98 \\
\hline 4559.243 & 29449.778 & ev & 1.5 & 7522.458 & od & 0.5 & $1.15 \pm 0.19$ & -1.84 \\
\hline 4560.280 & 29263.338 & od & 5.5 & 7341.007 & ev & 5.5 & $40.7 \pm 2.1$ & 0.18 \\
\hline 4560.958 & 27432.782 & od & 4.5 & 5513.709 & ev & 5.5 & $17.8 \pm 1.0$ & -0.26 \\
\hline 4562.359 & 25766.355 & od & 4.5 & 3854.012 & ev & 3.5 & $51.8 \pm 2.6$ & 0.21 \\
\hline 4568.036 & 30166.057 & ev & 3.5 & 8280.946 & od & 2.5 & $0.40 \pm 0.04$ & -2.00 \\
\hline 4569.670 & 32802.165 & ev & 5.5 & 10924.876 & od & 4.5 & $5.8 \pm 0.5$ & -0.66 \\
\hline 4572.278 & 27378.515 & od & 5.5 & 5513.709 & ev & 5.5 & $43.7 \pm 2.2$ & 0.22 \\
\hline 4576.799 & 30245.878 & ev & 4.5 & 8402.668 & od & 3.5 & $0.80 \pm 0.08$ & -1.60 \\
\hline 4582.050 & 29994.041 & ev & 2.5 & 8175.863 & od & 2.5 & $0.52 \pm 0.06$ & -2.00 \\
\hline 4582.406 & 28337.814 & ev & 2.5 & 6521.332 & od & 1.5 & $6.0 \pm 0.4$ & -0.95 \\
\hline 4582.499 & 27432.782 & od & 4.5 & 5616.739 & ev & 4.5 & $14.1 \pm 0.8$ & -0.35 \\
\hline 4588.416 & 28337.814 & ev & 2.5 & 6549.908 & od & 2.5 & $2.93 \pm 0.21$ & -1.26 \\
\hline 4589.374 & 24663.053 & ev & 4.5 & 2879.695 & od & 5.5 & $0.336 \pm 0.027$ & -1.97 \\
\hline 4591.116 & 30702.610 & ev & 4.5 & 8927.514 & od & 5.5 & $12.2 \pm 1.0$ & -0.41 \\
\hline 4593.926 & 27378.515 & od & 5.5 & 5616.739 & ev & 4.5 & $31.1 \pm 1.6$ & 0.07 \\
\hline 4596.928 & 28297.473 & ev & 3.5 & 6549.908 & od & 2.5 & $1.16 \pm 0.12$ & -1.53 \\
\hline 4601.374 & 32372.621 & od & 4.5 & 10646.070 & ev & 5.5 & $4.9 \pm 0.4$ & -0.81 \\
\hline 4604.226 & 29994.041 & ev & 2.5 & 8280.946 & od & 2.5 & $2.45 \pm 0.30$ & -1.33 \\
\hline 4606.400 & 29043.854 & od & 6.5 & 7341.007 & ev & 5.5 & $19.5 \pm 1.0$ & -0.06 \\
\hline 4613.033 & 28730.712 & ev & 3.5 & 7059.072 & od & 4.5 & $2.34 \pm 0.22$ & -1.22 \\
\hline 4613.528 & 32372.621 & od & 4.5 & 10703.305 & ev & 4.5 & $0.92 \pm 0.13$ & -1.53 \\
\hline 4618.930 & 29166.597 & ev & 4.5 & 7522.622 & od & 5.5 & $0.99 \pm 0.09$ & -1.50 \\
\hline 4621.743 & 30829.124 & ev & 3.5 & 9198.326 & od & 3.5 & $0.86 \pm 0.11$ & -1.66 \\
\hline 4623.477 & 25945.396 & ev & 3.5 & 4322.708 & od & 2.5 & $0.53 \pm 0.03$ & -1.87 \\
\hline 4625.290 & 25359.686 & ev & 2.5 & 3745.475 & od & 1.5 & $0.41 \pm 0.03$ & -2.10 \\
\hline 4628.161 & 25766.355 & od & 4.5 & 4165.550 & ev & 4.5 & $42.5 \pm 2.2$ & 0.14 \\
\hline 4630.183 & 29994.041 & ev & 2.5 & 8402.668 & od & 3.5 & $0.14 \pm 0.03$ & -2.55 \\
\hline 4633.601 & 28634.516 & ev & 5.5 & 7059.072 & od & 4.5 & $1.58 \pm 0.23$ & -1.21 \\
\hline 4636.741 & 27379.949 & ev & 5.5 & 5819.113 & od & 4.5 & $0.66 \pm 0.07$ & -1.59 \\
\hline 4640.214 & 27934.638 & ev & 4.5 & 6389.942 & od & 4.5 & $0.341 \pm 0.027$ & -1.96 \\
\hline 4643.774 & 28730.712 & ev & 3.5 & 7202.529 & od & 2.5 & $1.03 \pm 0.10$ & -1.57 \\
\hline 4644.216 & 29807.078 & ev & 3.5 & 8280.946 & od & 2.5 & $5.1 \pm 0.4$ & -0.88 \\
\hline 4647.422 & 27187.047 & ev & 3.5 & 5675.763 & od & 4.5 & $2.04 \pm 0.18$ & -1.28 \\
\hline 4648.513 & 29908.904 & ev & 4.5 & 8402.668 & od & 3.5 & $0.51 \pm 0.05$ & -1.78 \\
\hline 4654.278 & 25681.488 & ev & 1.5 & 4201.893 & od & 1.5 & $8.7 \pm 0.6$ & -0.95 \\
\hline 4656.003 & 28730.712 & ev & 3.5 & 7259.075 & od & 3.5 & $2.9 \pm 0.4$ & -1.12 \\
\hline 4656.178 & 27187.047 & ev & 3.5 & 5716.216 & od & 3.5 & $0.85 \pm 0.10$ & -1.66 \\
\hline 4657.210 & 28725.148 & ev & 4.5 & 7259.075 & od & 3.5 & $1.59 \pm 0.18$ & -1.29 \\
\hline 4657.828 & 29281.374 & ev & 2.5 & 7818.147 & od & 1.5 & $2.22 \pm 0.17$ & -1.36 \\
\hline 4659.101 & 29735.413 & od & 4.5 & 8278.054 & ev & 5.5 & $0.25 \pm 0.03$ & -2.10 \\
\hline 4659.938 & 29166.597 & ev & 4.5 & 7713.089 & od & 4.5 & $2.16 \pm 0.22$ & -1.15 \\
\hline 4664.149 & 25945.396 & ev & 3.5 & 4511.257 & od & 2.5 & $0.41 \pm 0.07$ & -1.97 \\
\hline 4666.713 & 25945.396 & ev & 3.5 & 4523.033 & od & 4.5 & $1.00 \pm 0.15$ & -1.58 \\
\hline 4666.889 & 27811.496 & ev & 3.5 & 6389.942 & od & 4.5 & $0.41 \pm 0.05$ & -1.97 \\
\hline 4670.098 & 28685.758 & ev & 2.5 & 7278.922 & od & 1.5 & $3.4 \pm 0.3$ & -1.18 \\
\hline 4670.627 & 29807.078 & ev & 3.5 & 8402.668 & od & 3.5 & $0.67 \pm 0.09$ & -1.75 \\
\hline
\end{tabular}


Table 2

(Continued)

\begin{tabular}{|c|c|c|c|c|c|c|c|c|}
\hline$\lambda_{\text {air }}(\AA)$ & $E_{\text {upper }}\left(\mathrm{cm}^{-1}\right)$ & Parity & $J_{\text {upp }}$ & $E_{\text {lower }}\left(\mathrm{cm}^{-1}\right)$ & Parity & $J_{\text {low }}$ & $A$-value $\left(10^{6} \mathrm{~s}^{-1}\right)$ & $\log (g f)$ \\
\hline 4670.725 & 26841.384 & ev & 4.5 & 5437.422 & od & 3.5 & $3.08 \pm 0.19$ & -1.00 \\
\hline 4671.395 & 28634.516 & ev & 5.5 & 7233.627 & od & 5.5 & $0.76 \pm 0.13$ & -1.53 \\
\hline 4678.600 & 27187.047 & ev & 3.5 & 5819.113 & od & 4.5 & $1.17 \pm 0.11$ & -1.51 \\
\hline 4679.412 & 25359.686 & ev & 2.5 & 3995.460 & od & 3.5 & $0.57 \pm 0.08$ & -1.95 \\
\hline 4680.119 & 29892.677 & od & 3.5 & 8531.678 & ev & 3.5 & $12.6 \pm 1.0$ & -0.48 \\
\hline 4680.442 & 28327.071 & od & 5.5 & 6967.547 & ev & 6.5 & $1.57 \pm 0.10$ & -1.21 \\
\hline 4680.986 & 32372.621 & od & 4.5 & 11015.579 & ev & 3.5 & $5.4 \pm 0.4$ & -0.75 \\
\hline 4684.598 & 28634.516 & ev & 5.5 & 7293.938 & od & 6.5 & $10.3 \pm 0.8$ & -0.39 \\
\hline 4686.770 & 30134.910 & ev & 5.5 & 8804.224 & od & 4.5 & $3.38 \pm 0.28$ & -0.87 \\
\hline 4687.608 & 29750.547 & od & 5.5 & 8423.672 & ev & 6.5 & $0.85 \pm 0.08$ & -1.48 \\
\hline 4689.479 & 30245.878 & ev & 4.5 & 8927.514 & od & 5.5 & $2.47 \pm 0.17$ & -1.09 \\
\hline 4690.160 & 28327.071 & od & 5.5 & 7011.804 & ev & 4.5 & $1.48 \pm 0.11$ & -1.23 \\
\hline 4690.479 & 29591.873 & od & 6.5 & 8278.054 & ev & 5.5 & $2.15 \pm 0.16$ & -1.00 \\
\hline 4692.009 & 27249.669 & ev & 2.5 & 5942.798 & od & 3.5 & $2.27 \pm 0.21$ & -1.35 \\
\hline 4694.320 & 27934.638 & ev & 4.5 & 6638.258 & od & 4.5 & $0.47 \pm 0.05$ & -1.81 \\
\hline 4694.872 & 27811.496 & ev & 3.5 & 6517.619 & od & 2.5 & $2.91 \pm 0.22$ & -1.11 \\
\hline 4695.375 & 29994.041 & ev & 2.5 & 8702.444 & od & 1.5 & $1.20 \pm 0.09$ & -1.62 \\
\hline 4698.886 & 28334.756 & ev & 4.5 & 7059.072 & od & 4.5 & $0.36 \pm 0.05$ & -1.93 \\
\hline 4702.002 & 27811.496 & ev & 3.5 & 6549.908 & od & 2.5 & $2.34 \pm 0.18$ & -1.21 \\
\hline 4707.932 & 28327.071 & od & 5.5 & 7092.265 & ev & 5.5 & $1.81 \pm 0.17$ & -1.14 \\
\hline 4710.198 & 26900.354 & ev & 3.5 & 5675.763 & od & 4.5 & $0.61 \pm 0.08$ & -1.79 \\
\hline 4714.017 & 30134.910 & ev & 5.5 & 8927.514 & od & 5.5 & $11.7 \pm 0.7$ & -0.33 \\
\hline 4714.831 & 29735.413 & od & 4.5 & 8531.678 & ev & 3.5 & $7.5 \pm 0.5$ & -0.60 \\
\hline 4717.881 & 26841.384 & ev & 4.5 & 5651.357 & od & 5.5 & $2.15 \pm 0.25$ & -1.14 \\
\hline 4719.193 & 26900.354 & ev & 3.5 & 5716.216 & od & 3.5 & $0.29 \pm 0.04$ & -2.12 \\
\hline 4722.293 & 25681.488 & ev & 1.5 & 4511.257 & od & 2.5 & $2.55 \pm 0.17$ & -1.47 \\
\hline 4722.746 & 29591.873 & od & 6.5 & 8423.672 & ev & 6.5 & $0.98 \pm 0.07$ & -1.34 \\
\hline 4723.321 & 26841.384 & ev & 4.5 & 5675.763 & od & 4.5 & $1.25 \pm 0.08$ & -1.38 \\
\hline 4730.101 & 28337.814 & ev & 2.5 & 7202.529 & od & 2.5 & $6.4 \pm 0.4$ & -0.89 \\
\hline 4732.366 & 26841.384 & ev & 4.5 & 5716.216 & od & 3.5 & $0.83 \pm 0.07$ & -1.55 \\
\hline 4733.835 & 29892.677 & od & 3.5 & 8774.064 & ev & 4.5 & $1.77 \pm 0.17$ & -1.32 \\
\hline 4737.271 & 29892.677 & od & 3.5 & 8789.380 & ev & 2.5 & $29.0 \pm 1.8$ & -0.11 \\
\hline 4739.147 & 28297.473 & ev & 3.5 & 7202.529 & od & 2.5 & $3.11 \pm 0.24$ & -1.08 \\
\hline 4742.227 & 26900.354 & ev & 3.5 & 5819.113 & od & 4.5 & $0.68 \pm 0.07$ & -1.74 \\
\hline 4744.944 & 24663.053 & ev & 4.5 & 3593.882 & od & 4.5 & $1.90 \pm 0.12$ & -1.19 \\
\hline 4747.260 & 28337.814 & ev & 2.5 & 7278.922 & od & 1.5 & $0.80 \pm 0.10$ & -1.79 \\
\hline 4749.234 & 30829.124 & ev & 3.5 & 9778.986 & od & 2.5 & $2.05 \pm 0.20$ & -1.26 \\
\hline 4757.841 & 28725.148 & ev & 4.5 & 7713.089 & od & 4.5 & $5.7 \pm 0.4$ & -0.71 \\
\hline 4759.927 & 29807.078 & ev & 3.5 & 8804.224 & od & 4.5 & $2.63 \pm 0.17$ & -1.14 \\
\hline 4762.840 & 27379.949 & ev & 5.5 & 6389.942 & od & 4.5 & $0.194 \pm 0.021$ & -2.10 \\
\hline 4763.735 & 28327.071 & od & 5.5 & 7341.007 & ev & 5.5 & $0.145 \pm 0.027$ & -2.23 \\
\hline 4763.912 & 29263.338 & od & 5.5 & 8278.054 & ev & 5.5 & $3.53 \pm 0.23$ & -0.84 \\
\hline 4768.791 & 27975.619 & od & 4.5 & 7011.804 & ev & 4.5 & $4.7 \pm 0.4$ & -0.80 \\
\hline 4769.782 & 24663.053 & ev & 4.5 & 3703.594 & od & 3.5 & $0.245 \pm 0.025$ & -2.08 \\
\hline 4773.941 & 28396.150 & od & 2.5 & 7454.951 & ev & 1.5 & $20.0 \pm 1.2$ & -0.39 \\
\hline 4775.463 & 25945.396 & ev & 3.5 & 5010.870 & od & 2.5 & $1.20 \pm 0.19$ & -1.49 \\
\hline 4787.165 & 27975.619 & od & 4.5 & 7092.265 & ev & 5.5 & $1.37 \pm 0.15$ & -1.33 \\
\hline 4788.230 & 29281.374 & ev & 2.5 & 8402.668 & od & 3.5 & $1.73 \pm 0.19$ & -1.45 \\
\hline 4789.682 & 26841.384 & ev & 4.5 & 5969.007 & od & 5.5 & $0.93 \pm 0.13$ & -1.49 \\
\hline 4792.944 & 30637.157 & ev & 2.5 & 9778.986 & od & 2.5 & $0.87 \pm 0.13$ & -1.75 \\
\hline 4795.184 & 25359.686 & ev & 2.5 & 4511.257 & od & 2.5 & $1.89 \pm 0.22$ & -1.41 \\
\hline 4795.554 & 28725.148 & ev & 4.5 & 7878.328 & od & 3.5 & $2.13 \pm 0.25$ & -1.13 \\
\hline 4797.343 & 29892.677 & od & 3.5 & 9053.629 & ev & 3.5 & $0.49 \pm 0.05$ & -1.87 \\
\hline 4797.426 & 29735.413 & od & 4.5 & 8896.729 & ev & 5.5 & $1.08 \pm 0.07$ & -1.43 \\
\hline 4797.850 & 25681.488 & ev & 1.5 & 4844.644 & od & 1.5 & $0.27 \pm 0.03$ & -2.43 \\
\hline 4804.633 & 28685.758 & ev & 2.5 & 7878.328 & od & 3.5 & $0.76 \pm 0.08$ & -1.80 \\
\hline 4812.505 & 27835.233 & ev & 1.5 & 7061.838 & od & 0.5 & $1.97 \pm 0.19$ & -1.56 \\
\hline 4835.674 & 28396.150 & od & 2.5 & 7722.285 & ev & 2.5 & $5.8 \pm 0.4$ & -0.92 \\
\hline 4847.914 & 28334.756 & ev & 4.5 & 7713.089 & od & 4.5 & $5.0 \pm 0.5$ & -0.75 \\
\hline 4848.263 & 29043.854 & od & 6.5 & 8423.672 & ev & 6.5 & $0.97 \pm 0.07$ & -1.32 \\
\hline 4850.902 & 27811.496 & ev & 3.5 & 7202.529 & od & 2.5 & $1.52 \pm 0.15$ & -1.37 \\
\hline 4858.729 & 29892.677 & od & 3.5 & 9316.912 & ev & 3.5 & $4.9 \pm 0.4$ & -0.86 \\
\hline 4861.821 & 25681.488 & ev & 1.5 & 5118.806 & od & 2.5 & $0.42 \pm 0.06$ & -2.23 \\
\hline 4863.673 & 28730.712 & ev & 3.5 & 8175.863 & od & 2.5 & $0.95 \pm 0.11$ & -1.57 \\
\hline 4863.838 & 30829.124 & ev & 3.5 & 10274.971 & od & 3.5 & $1.80 \pm 0.24$ & -1.29 \\
\hline 4873.999 & 29438.817 & ev & 5.5 & 8927.514 & od & 5.5 & $4.7 \pm 0.5$ & -0.69 \\
\hline
\end{tabular}


Table 2

(Continued)

\begin{tabular}{|c|c|c|c|c|c|c|c|c|}
\hline$\lambda_{\text {air }}(\AA)$ & $E_{\text {upper }}\left(\mathrm{cm}^{-1}\right)$ & Parity & $J_{\text {upp }}$ & $E_{\text {lower }}\left(\mathrm{cm}^{-1}\right)$ & Parity & $J_{\text {low }}$ & $A$-value $\left(10^{6} \mathrm{~s}^{-1}\right)$ & $\log (g f)$ \\
\hline 4874.211 & 26900.354 & ev & 3.5 & 6389.942 & od & 4.5 & $0.25 \pm 0.05$ & -2.14 \\
\hline 4882.463 & 32802.165 & ev & 5.5 & 12326.417 & od & 6.5 & $35.8 \pm 2.4$ & 0.19 \\
\hline 4887.074 & 28334.756 & ev & 4.5 & 7878.328 & od & 3.5 & $0.31 \pm 0.05$ & -1.96 \\
\hline 4890.351 & 30166.057 & ev & 3.5 & 9723.335 & od & 4.5 & $1.71 \pm 0.16$ & -1.31 \\
\hline 4895.558 & 27432.782 & od & 4.5 & 7011.804 & ev & 4.5 & $0.72 \pm 0.04$ & -1.59 \\
\hline 4895.998 & 28297.473 & $\mathrm{ev}$ & 3.5 & 7878.328 & od & 3.5 & $1.30 \pm 0.10$ & -1.43 \\
\hline 4896.152 & 29735.413 & od & 4.5 & 9316.912 & ev & 3.5 & $0.34 \pm 0.05$ & -1.91 \\
\hline 4897.959 & 27378.515 & od & 5.5 & 6967.547 & ev & 6.5 & $0.292 \pm 0.028$ & -1.90 \\
\hline 4901.396 & 24663.053 & ev & 4.5 & 4266.397 & od & 3.5 & $0.54 \pm 0.08$ & -1.71 \\
\hline 4903.700 & 30166.057 & ev & 3.5 & 9778.986 & od & 2.5 & $1.62 \pm 0.19$ & -1.33 \\
\hline 4912.919 & 25359.686 & ev & 2.5 & 5010.870 & od & 2.5 & $0.20 \pm 0.03$ & -2.35 \\
\hline 4914.607 & 30065.164 & ev & 3.5 & 9723.335 & od & 4.5 & $1.59 \pm 0.18$ & -1.34 \\
\hline 4914.924 & 27432.782 & od & 4.5 & 7092.265 & ev & 5.5 & $4.3 \pm 0.3$ & -0.81 \\
\hline 4917.939 & 28730.712 & ev & 3.5 & 8402.668 & od & 3.5 & $0.38 \pm 0.04$ & -1.95 \\
\hline 4928.072 & 27378.515 & od & 5.5 & 7092.265 & ev & 5.5 & $1.15 \pm 0.08$ & -1.30 \\
\hline 4932.112 & 25945.396 & ev & 3.5 & 5675.763 & od & 4.5 & $0.30 \pm 0.04$ & -2.06 \\
\hline 4946.624 & 30245.878 & ev & 4.5 & 10035.711 & od & 5.5 & $2.00 \pm 0.19$ & -1.14 \\
\hline 4952.134 & 30829.124 & ev & 3.5 & 10641.442 & od & 2.5 & $0.37 \pm 0.06$ & -1.96 \\
\hline 4962.103 & 29043.854 & od & 6.5 & 8896.729 & ev & 5.5 & $0.39 \pm 0.04$ & -1.69 \\
\hline 4967.241 & 25945.396 & ev & 3.5 & 5819.113 & od & 4.5 & $0.29 \pm 0.04$ & -2.07 \\
\hline 4968.395 & 28297.473 & ev & 3.5 & 8175.863 & od & 2.5 & $1.30 \pm 0.12$ & -1.41 \\
\hline 4977.201 & 27379.949 & ev & 5.5 & 7293.938 & od & 6.5 & $2.26 \pm 0.27$ & -1.00 \\
\hline 4984.433 & 28337.814 & ev & 2.5 & 8280.946 & od & 2.5 & $4.2 \pm 0.4$ & -1.02 \\
\hline 4991.014 & 31340.393 & od & 6.5 & 11309.972 & ev & 7.5 & $4.0 \pm 0.3$ & -0.68 \\
\hline 4994.478 & 28297.473 & ev & 3.5 & 8280.946 & od & 2.5 & $2.7 \pm 0.3$ & -1.09 \\
\hline 4994.727 & 29794.517 & ev & 3.5 & 9778.986 & od & 2.5 & $5.4 \pm 0.5$ & -0.79 \\
\hline 4997.956 & 25945.396 & ev & 3.5 & 5942.798 & od & 3.5 & $0.148 \pm 0.018$ & -2.35 \\
\hline 5000.958 & 27249.669 & ev & 2.5 & 7259.075 & od & 3.5 & $0.85 \pm 0.08$ & -1.72 \\
\hline 5002.779 & 28685.758 & ev & 2.5 & 8702.444 & od & 1.5 & $6.6 \pm 0.6$ & -0.83 \\
\hline 5011.759 & 28396.150 & od & 2.5 & 8448.641 & ev & 2.5 & $14.3 \pm 1.0$ & -0.49 \\
\hline 5015.138 & 27812.398 & ev & 2.5 & 7878.328 & od & 3.5 & $0.67 \pm 0.06$ & -1.82 \\
\hline 5015.365 & 27811.496 & ev & 3.5 & 7878.328 & od & 3.5 & $0.41 \pm 0.05$ & -1.91 \\
\hline 5018.448 & 28725.148 & ev & 4.5 & 8804.224 & od & 4.5 & $0.30 \pm 0.05$ & -1.95 \\
\hline 5022.652 & 30829.124 & ev & 3.5 & 10924.876 & od & 4.5 & $0.63 \pm 0.10$ & -1.72 \\
\hline 5022.867 & 28327.071 & od & 5.5 & 8423.672 & ev & 6.5 & $9.2 \pm 0.7$ & -0.38 \\
\hline 5027.339 & 31340.393 & od & 6.5 & 11454.701 & ev & 6.5 & $3.2 \pm 0.5$ & -0.77 \\
\hline 5028.263 & 31340.393 & od & 6.5 & 11458.353 & ev & 5.5 & $2.68 \pm 0.26$ & -0.85 \\
\hline 5037.800 & 27975.619 & od & 4.5 & 8131.217 & ev & 4.5 & $7.0 \pm 0.6$ & -0.57 \\
\hline 5039.273 & 30637.157 & ev & 2.5 & 10798.555 & od & 2.5 & $1.43 \pm 0.14$ & -1.49 \\
\hline 5044.023 & 29591.873 & od & 6.5 & 9771.956 & ev & 7.5 & $13.5 \pm 1.0$ & -0.14 \\
\hline 5049.701 & 28725.148 & ev & 4.5 & 8927.514 & od & 5.5 & $0.37 \pm 0.04$ & -1.85 \\
\hline 5072.924 & 28634.516 & ev & 5.5 & 8927.514 & od & 5.5 & $1.56 \pm 0.20$ & -1.14 \\
\hline 5075.355 & 27975.619 & od & 4.5 & 8278.054 & ev & 5.5 & $11.5 \pm 0.8$ & -0.35 \\
\hline 5083.279 & 27379.949 & ev & 5.5 & 7713.089 & od & 4.5 & $0.50 \pm 0.06$ & -1.64 \\
\hline 5085.216 & 27835.233 & ev & 1.5 & 8175.863 & od & 2.5 & $0.52 \pm 0.06$ & -2.10 \\
\hline 5089.487 & 28345.313 & ev & 0.5 & 8702.444 & od & 1.5 & $1.62 \pm 0.21$ & -1.90 \\
\hline 5105.227 & 26841.384 & ev & 4.5 & 7259.075 & od & 3.5 & $1.22 \pm 0.11$ & -1.32 \\
\hline 5106.217 & 29892.677 & od & 3.5 & 10314.162 & ev & 4.5 & $0.57 \pm 0.08$ & -1.75 \\
\hline 5112.543 & 27835.233 & ev & 1.5 & 8280.946 & od & 2.5 & $0.94 \pm 0.12$ & -1.83 \\
\hline 5117.946 & 29591.873 & od & 6.5 & 10058.226 & ev & 6.5 & $2.8 \pm 0.3$ & -0.81 \\
\hline 5118.349 & 29807.078 & ev & 3.5 & 10274.971 & od & 3.5 & $0.96 \pm 0.11$ & -1.52 \\
\hline 5141.737 & 29166.597 & ev & 4.5 & 9723.335 & od & 4.5 & $0.40 \pm 0.03$ & -1.80 \\
\hline 5144.844 & 27249.669 & ev & 2.5 & 7818.147 & od & 1.5 & $1.12 \pm 0.16$ & -1.58 \\
\hline 5145.156 & 28327.071 & od & 5.5 & 8896.729 & ev & 5.5 & $1.93 \pm 0.22$ & -1.04 \\
\hline 5146.910 & 30065.164 & ev & 3.5 & 10641.442 & od & 2.5 & $1.17 \pm 0.13$ & -1.43 \\
\hline 5147.565 & 29735.413 & od & 4.5 & 10314.162 & ev & 4.5 & $12.5 \pm 0.9$ & -0.30 \\
\hline 5150.860 & 27811.496 & ev & 3.5 & 8402.668 & od & 3.5 & $0.58 \pm 0.07$ & -1.73 \\
\hline 5163.547 & 29449.778 & ev & 1.5 & 10088.640 & od & 1.5 & $0.47 \pm 0.09$ & -2.12 \\
\hline 5187.458 & 29043.854 & od & 6.5 & 9771.956 & ev & 7.5 & $26.2 \pm 1.8$ & 0.17 \\
\hline 5191.633 & 26268.203 & od & 3.5 & 7011.804 & ev & 4.5 & $7.8 \pm 0.6$ & -0.60 \\
\hline 5192.092 & 32372.621 & od & 4.5 & 13117.922 & ev & 4.5 & $1.55 \pm 0.24$ & -1.20 \\
\hline 5208.108 & 29994.041 & ev & 2.5 & 10798.555 & od & 2.5 & $0.42 \pm 0.08$ & -1.99 \\
\hline 5210.339 & 26900.354 & ev & 3.5 & 7713.089 & od & 4.5 & $2.64 \pm 0.30$ & -1.07 \\
\hline 5216.701 & 25681.488 & ev & 1.5 & 6517.619 & od & 2.5 & $1.17 \pm 0.13$ & -1.72 \\
\hline 5217.711 & 25681.488 & ev & 1.5 & 6521.332 & od & 1.5 & $1.07 \pm 0.15$ & -1.76 \\
\hline 5225.505 & 25681.488 & ev & 1.5 & 6549.908 & od & 2.5 & $2.6 \pm 0.3$ & -1.38 \\
\hline
\end{tabular}


Table 2

(Continued)

\begin{tabular}{|c|c|c|c|c|c|c|c|c|}
\hline$\lambda_{\text {air }}(\AA)$ & $E_{\text {upper }}\left(\mathrm{cm}^{-1}\right)$ & Parity & $J_{\text {upp }}$ & $E_{\text {lower }}\left(\mathrm{cm}^{-1}\right)$ & Parity & $J_{\text {low }}$ & $A$-value $\left(10^{6} \mathrm{~s}^{-1}\right)$ & $\log (g f)$ \\
\hline 5226.402 & 26841.384 & ev & 4.5 & 7713.089 & od & 4.5 & $1.08 \pm 0.16$ & -1.35 \\
\hline 5232.918 & 29750.547 & od & 5.5 & 10646.070 & ev & 5.5 & $10.0 \pm 0.9$ & -0.31 \\
\hline 5234.019 & 27378.515 & od & 5.5 & 8278.054 & ev & 5.5 & $3.3 \pm 0.3$ & -0.79 \\
\hline 5237.067 & 29735.413 & od & 4.5 & 10646.070 & ev & 5.5 & $5.8 \pm 0.5$ & -0.62 \\
\hline 5241.333 & 27249.669 & ev & 2.5 & 8175.863 & od & 2.5 & $0.73 \pm 0.08$ & -1.75 \\
\hline 5241.777 & 29892.677 & od & 3.5 & 10820.486 & ev & 2.5 & $2.52 \pm 0.26$ & -1.08 \\
\hline 5252.817 & 29735.413 & od & 4.5 & 10703.305 & ev & 4.5 & $1.72 \pm 0.15$ & -1.15 \\
\hline 5262.938 & 29449.778 & ev & 1.5 & 10454.272 & od & 1.5 & $1.18 \pm 0.25$ & -1.71 \\
\hline 5265.506 & 29994.041 & ev & 2.5 & 11007.799 & od & 1.5 & $0.26 \pm 0.05$ & -2.20 \\
\hline 5265.677 & 29043.854 & od & 6.5 & 10058.226 & ev & 6.5 & $10.0 \pm 0.7$ & -0.23 \\
\hline 5274.229 & 27378.515 & od & 5.5 & 8423.672 & ev & 6.5 & $26.8 \pm 1.6$ & 0.13 \\
\hline 5276.457 & 24663.053 & ev & 4.5 & 5716.216 & od & 3.5 & $0.102 \pm 0.010$ & -2.37 \\
\hline 5283.386 & 27975.619 & od & 4.5 & 9053.629 & ev & 3.5 & $1.06 \pm 0.09$ & -1.35 \\
\hline 5294.855 & 29750.547 & od & 5.5 & 10869.541 & ev & 4.5 & $0.43 \pm 0.05$ & -1.66 \\
\hline 5295.155 & 30829.124 & ev & 3.5 & 11949.189 & od & 3.5 & $0.53 \pm 0.09$ & -1.75 \\
\hline 5304.408 & 27249.669 & ev & 2.5 & 8402.668 & od & 3.5 & $0.52 \pm 0.07$ & -1.88 \\
\hline 5327.492 & 29449.778 & ev & 1.5 & 10684.441 & od & 0.5 & $1.00 \pm 0.15$ & -1.77 \\
\hline 5330.556 & 25766.355 & od & 4.5 & 7011.804 & ev & 4.5 & $9.3 \pm 0.7$ & -0.40 \\
\hline 5350.020 & 25945.396 & ev & 3.5 & 7259.075 & od & 3.5 & $0.127 \pm 0.017$ & -2.36 \\
\hline 5353.524 & 25766.355 & od & 4.5 & 7092.265 & ev & 5.5 & $28.8 \pm 1.7$ & 0.09 \\
\hline 5369.864 & 29263.338 & od & 5.5 & 10646.070 & ev & 5.5 & $0.20 \pm 0.04$ & -1.98 \\
\hline 5383.812 & 30829.124 & ev & 3.5 & 12260.088 & od & 3.5 & $0.40 \pm 0.06$ & -1.86 \\
\hline 5386.773 & 28337.814 & ev & 2.5 & 9778.986 & od & 2.5 & $7.5 \pm 0.6$ & -0.71 \\
\hline 5390.523 & 26268.203 & od & 3.5 & 7722.285 & ev & 2.5 & $0.35 \pm 0.04$ & -1.91 \\
\hline 5393.392 & 27432.782 & od & 4.5 & 8896.729 & ev & 5.5 & $19.9 \pm 1.3$ & -0.06 \\
\hline 5398.508 & 28297.473 & ev & 3.5 & 9778.986 & od & 2.5 & $1.60 \pm 0.13$ & -1.25 \\
\hline 5409.229 & 27378.515 & od & 5.5 & 8896.729 & ev & 5.5 & $9.8 \pm 0.7$ & -0.29 \\
\hline 5413.051 & 29794.517 & ev & 3.5 & 11325.781 & od & 2.5 & $0.51 \pm 0.06$ & -1.75 \\
\hline 5433.927 & 29043.854 & od & 6.5 & 10646.070 & ev & 5.5 & $0.53 \pm 0.04$ & -1.48 \\
\hline 5435.105 & 29263.338 & od & 5.5 & 10869.541 & ev & 4.5 & $0.361 \pm 0.029$ & -1.72 \\
\hline 5459.193 & 31340.393 & od & 6.5 & 13027.758 & ev & 6.5 & $7.5 \pm 0.9$ & -0.33 \\
\hline 5464.203 & 29750.547 & od & 5.5 & 11454.701 & ev & 6.5 & $5.8 \pm 0.6$ & -0.50 \\
\hline 5468.371 & 29591.873 & od & 6.5 & 11309.972 & ev & 7.5 & $13.4 \pm 1.1$ & -0.07 \\
\hline 5505.941 & 25359.686 & ev & 2.5 & 7202.529 & od & 2.5 & $0.54 \pm 0.05$ & -1.84 \\
\hline 5512.064 & 26268.203 & od & 3.5 & 8131.217 & ev & 4.5 & $11.3 \pm 1.0$ & -0.39 \\
\hline 5513.118 & 29591.873 & od & 6.5 & 11458.353 & ev & 5.5 & $1.47 \pm 0.19$ & -1.03 \\
\hline 5518.489 & 27432.782 & od & 4.5 & 9316.912 & ev & 3.5 & $4.9 \pm 0.4$ & -0.65 \\
\hline 5524.450 & 32372.621 & od & 4.5 & 14276.298 & ev & 5.5 & $2.44 \pm 0.27$ & -0.95 \\
\hline 5547.079 & 28297.473 & ev & 3.5 & 10274.971 & od & 3.5 & $0.194 \pm 0.027$ & -2.15 \\
\hline 5550.033 & 28327.071 & od & 5.5 & 10314.162 & ev & 4.5 & $2.00 \pm 0.20$ & -0.96 \\
\hline 5561.445 & 29735.413 & od & 4.5 & 11759.467 & ev & 5.5 & $2.83 \pm 0.26$ & -0.88 \\
\hline 5610.253 & 26268.203 & od & 3.5 & 8448.641 & ev & 2.5 & $5.9 \pm 0.5$ & -0.65 \\
\hline 5613.694 & 29263.338 & od & 5.5 & 11454.701 & ev & 6.5 & $4.1 \pm 0.4$ & -0.64 \\
\hline 5637.359 & 29043.854 & od & 6.5 & 11309.972 & ev & 7.5 & $3.6 \pm 0.3$ & -0.62 \\
\hline 5654.219 & 28327.071 & od & 5.5 & 10646.070 & ev & 5.5 & $0.248 \pm 0.028$ & -1.85 \\
\hline 5659.517 & 25945.396 & ev & 3.5 & 8280.946 & od & 2.5 & $0.262 \pm 0.027$ & -2.00 \\
\hline 5660.476 & 27975.619 & od & 4.5 & 10314.162 & ev & 4.5 & $0.51 \pm 0.04$ & -1.61 \\
\hline 5667.960 & 29735.413 & od & 4.5 & 12097.276 & ev & 3.5 & $1.56 \pm 0.16$ & -1.12 \\
\hline 5683.745 & 29043.854 & od & 6.5 & 11454.701 & ev & 6.5 & $2.03 \pm 0.16$ & -0.86 \\
\hline 5684.925 & 29043.854 & od & 6.5 & 11458.353 & ev & 5.5 & $0.77 \pm 0.08$ & -1.28 \\
\hline 5733.693 & 29892.677 & od & 3.5 & 12456.746 & ev & 3.5 & $1.59 \pm 0.17$ & -1.20 \\
\hline 5768.891 & 27975.619 & od & 4.5 & 10646.070 & ev & 5.5 & $6.0 \pm 0.5$ & -0.52 \\
\hline 5771.975 & 27378.515 & od & 5.5 & 10058.226 & ev & 6.5 & $1.38 \pm 0.12$ & -1.08 \\
\hline 5788.007 & 27975.619 & od & 4.5 & 10703.305 & ev & 4.5 & $0.85 \pm 0.12$ & -1.37 \\
\hline 5822.479 & 27811.496 & ev & 3.5 & 10641.442 & od & 2.5 & $0.29 \pm 0.05$ & -1.92 \\
\hline 5832.290 & 25945.396 & ev & 3.5 & 8804.224 & od & 4.5 & $0.54 \pm 0.06$ & -1.65 \\
\hline 5839.973 & 27432.782 & od & 4.5 & 10314.162 & ev & 4.5 & $0.23 \pm 0.05$ & -1.92 \\
\hline 5842.096 & 29438.817 & ev & 5.5 & 12326.417 & od & 6.5 & $0.58 \pm 0.06$ & -1.45 \\
\hline 5858.546 & 27378.515 & od & 5.5 & 10314.162 & ev & 4.5 & $1.08 \pm 0.19$ & -1.18 \\
\hline 5895.679 & 28297.473 & ev & 3.5 & 11340.598 & od & 3.5 & $0.44 \pm 0.06$ & -1.73 \\
\hline 5898.083 & 24663.053 & ev & 4.5 & 7713.089 & od & 4.5 & $0.53 \pm 0.06$ & -1.56 \\
\hline 5959.688 & 29892.677 & od & 3.5 & 13117.922 & ev & 4.5 & $4.8 \pm 0.5$ & -0.69 \\
\hline 5968.060 & 31155.623 & ev & 6.5 & 14404.400 & od & 7.5 & $1.33 \pm 0.13$ & -1.00 \\
\hline 5975.818 & 27432.782 & od & 4.5 & 10703.305 & ev & 4.5 & $6.6 \pm 0.6$ & -0.45 \\
\hline 5995.265 & 27378.515 & od & 5.5 & 10703.305 & ev & 4.5 & $1.62 \pm 0.17$ & -0.98 \\
\hline 5995.448 & 29892.677 & od & 3.5 & 13217.976 & ev & 3.5 & $2.01 \pm 0.19$ & -1.06 \\
\hline
\end{tabular}


Table 2

(Continued)

\begin{tabular}{|c|c|c|c|c|c|c|c|c|}
\hline$\lambda_{\text {air }}(\AA)$ & $E_{\text {upper }}\left(\mathrm{cm}^{-1}\right)$ & Parity & $J_{\text {upp }}$ & $E_{\text {lower }}\left(\mathrm{cm}^{-1}\right)$ & Parity & $J_{\text {low }}$ & $A$-value $\left(10^{6} \mathrm{~s}^{-1}\right)$ & $\log (g f)$ \\
\hline 6013.568 & 29892.677 & od & 3.5 & 13268.218 & ev & 2.5 & $1.72 \pm 0.23$ & -1.13 \\
\hline 6034.205 & 28327.071 & od & 5.5 & 11759.467 & ev & 5.5 & $3.5 \pm 0.4$ & -0.64 \\
\hline 6035.476 & 29591.873 & od & 6.5 & 13027.758 & ev & 6.5 & $2.37 \pm 0.25$ & -0.74 \\
\hline 6043.373 & 26268.203 & od & 3.5 & 9725.733 & ev & 3.5 & $7.5 \pm 0.8$ & -0.48 \\
\hline 6069.634 & 27811.496 & ev & 3.5 & 11340.598 & od & 3.5 & $0.76 \pm 0.11$ & -1.47 \\
\hline 6101.243 & 28334.756 & ev & 4.5 & 11949.189 & od & 3.5 & $0.29 \pm 0.04$ & -1.79 \\
\hline 6108.747 & 29892.677 & od & 3.5 & 13527.239 & ev & 4.5 & $4.2 \pm 0.5$ & -0.73 \\
\hline 6183.954 & 25945.396 & ev & 3.5 & 9778.986 & od & 2.5 & $0.89 \pm 0.10$ & -1.39 \\
\hline 6225.397 & 31340.393 & od & 6.5 & 15281.602 & ev & 6.5 & $0.49 \pm 0.05$ & -1.40 \\
\hline 6232.448 & 25766.355 & od & 4.5 & 9725.733 & ev & 3.5 & $2.2 \pm 0.3$ & -0.89 \\
\hline 6272.026 & 28396.150 & od & 2.5 & 12456.746 & ev & 3.5 & $11.1 \pm 1.1$ & -0.40 \\
\hline 6296.145 & 27975.619 & od & 4.5 & 12097.276 & ev & 3.5 & $0.66 \pm 0.08$ & -1.41 \\
\hline 6371.109 & 28396.150 & od & 2.5 & 12704.634 & ev & 1.5 & $6.6 \pm 0.7$ & -0.62 \\
\hline 6428.509 & 27811.496 & ev & 3.5 & 12260.088 & od & 3.5 & $0.35 \pm 0.05$ & -1.77 \\
\hline 6441.986 & 27975.619 & od & 4.5 & 12456.746 & ev & 3.5 & $0.42 \pm 0.05$ & -1.58 \\
\hline 6449.503 & 26841.384 & ev & 4.5 & 11340.598 & od & 3.5 & $0.126 \pm 0.016$ & -2.10 \\
\hline 6466.888 & 29735.413 & od & 4.5 & 14276.298 & ev & 5.5 & $3.6 \pm 0.5$ & -0.65 \\
\hline 6507.163 & 29750.547 & od & 5.5 & 14387.112 & ev & 4.5 & $1.39 \pm 0.15$ & -0.97 \\
\hline 6608.464 & 28396.150 & od & 2.5 & 13268.218 & ev & 2.5 & $0.52 \pm 0.07$ & -1.69 \\
\hline 6609.726 & 29750.547 & od & 5.5 & 14625.503 & ev & 5.5 & $0.69 \pm 0.08$ & -1.27 \\
\hline 6616.346 & 29735.413 & od & 4.5 & 14625.503 & ev & 5.5 & $0.85 \pm 0.10$ & -1.25 \\
\hline 6706.051 & 29735.413 & od & 4.5 & 14827.623 & ev & 3.5 & $1.92 \pm 0.24$ & -0.89 \\
\hline 6720.280 & 29263.338 & od & 5.5 & 14387.112 & ev & 4.5 & $1.52 \pm 0.13$ & -0.91 \\
\hline 6829.583 & 28297.473 & ev & 3.5 & 13659.329 & od & 4.5 & $0.134 \pm 0.022$ & -2.13 \\
\hline 6829.727 & 29263.338 & od & 5.5 & 14625.503 & ev & 5.5 & $1.68 \pm 0.15$ & -0.85 \\
\hline 6905.307 & 30637.157 & ev & 2.5 & 16159.536 & od & 3.5 & $0.84 \pm 0.11$ & -1.44 \\
\hline 6919.282 & 27975.619 & od & 4.5 & 13527.239 & ev & 4.5 & $1.41 \pm 0.14$ & -0.99 \\
\hline 6960.365 & 29892.677 & od & 3.5 & 15529.576 & ev & 2.5 & $0.19 \pm 0.04$ & -1.95 \\
\hline 6983.822 & 27432.782 & od & 4.5 & 13117.922 & ev & 4.5 & $0.88 \pm 0.12$ & -1.19 \\
\hline 7025.915 & 29794.517 & ev & 3.5 & 15565.420 & od & 2.5 & $0.45 \pm 0.07$ & -1.57 \\
\hline 7061.753 & 29750.547 & od & 5.5 & 15593.660 & ev & 6.5 & $8.1 \pm 0.9$ & -0.14 \\
\hline 7075.546 & 29263.338 & od & 5.5 & 15134.048 & ev & 4.5 & $0.27 \pm 0.04$ & -1.62 \\
\hline 7086.353 & 31340.393 & od & 6.5 & 17232.652 & ev & 7.5 & $12.6 \pm 1.4$ & 0.12 \\
\hline 7142.824 & 25945.396 & ev & 3.5 & 11949.189 & od & 3.5 & $0.128 \pm 0.018$ & -2.11 \\
\hline 7238.371 & 26268.203 & od & 3.5 & 12456.746 & ev & 3.5 & $1.96 \pm 0.20$ & -0.91 \\
\hline 7275.857 & 29892.677 & od & 3.5 & 16152.377 & ev & 3.5 & $0.133 \pm 0.022$ & -2.07 \\
\hline 7349.808 & 29794.517 & ev & 3.5 & 16192.466 & od & 4.5 & $0.16 \pm 0.03$ & -1.98 \\
\hline 7360.097 & 29735.413 & od & 4.5 & 16152.377 & ev & 3.5 & $0.51 \pm 0.07$ & -1.38 \\
\hline 7439.467 & 29892.677 & od & 3.5 & 16454.555 & ev & 2.5 & $0.33 \pm 0.05$ & -1.66 \\
\hline 7486.722 & 27835.233 & ev & 1.5 & 14481.930 & od & 2.5 & $0.33 \pm 0.06$ & -1.96 \\
\hline 7496.946 & 27432.782 & od & 4.5 & 14097.689 & ev & 3.5 & $0.70 \pm 0.08$ & -1.23 \\
\hline 7577.678 & 28327.071 & od & 5.5 & 15134.048 & ev & 4.5 & $0.43 \pm 0.05$ & -1.35 \\
\hline 7583.580 & 25945.396 & ev & 3.5 & 12762.641 & od & 4.5 & $0.065 \pm 0.011$ & -2.35 \\
\hline 7584.818 & 27432.782 & od & 4.5 & 14252.178 & ev & 3.5 & $0.39 \pm 0.05$ & -1.47 \\
\hline 7596.363 & 28396.150 & od & 2.5 & 15235.579 & ev & 1.5 & $0.66 \pm 0.10$ & -1.47 \\
\hline 7660.593 & 26268.203 & od & 3.5 & 13217.976 & ev & 3.5 & $0.27 \pm 0.03$ & -1.72 \\
\hline 7690.200 & 26268.203 & od & 3.5 & 13268.218 & ev & 2.5 & $0.63 \pm 0.07$ & -1.35 \\
\hline 7711.746 & 30134.910 & ev & 5.5 & 17171.245 & od & 5.5 & $0.29 \pm 0.04$ & -1.51 \\
\hline 7851.195 & 28327.071 & od & 5.5 & 15593.660 & ev & 6.5 & $1.99 \pm 0.25$ & -0.66 \\
\hline 7939.063 & 26268.203 & od & 3.5 & 13675.722 & ev & 2.5 & $0.58 \pm 0.07$ & -1.36 \\
\hline 8068.524 & 32372.621 & od & 4.5 & 19982.187 & ev & 4.5 & $1.45 \pm 0.24$ & -0.85 \\
\hline 8088.901 & 29591.873 & od & 6.5 & 17232.652 & ev & 7.5 & $1.47 \pm 0.19$ & -0.69 \\
\hline 8137.064 & 25945.396 & ev & 3.5 & 13659.329 & od & 4.5 & $0.139 \pm 0.020$ & -1.96 \\
\hline 8233.415 & 28334.756 & ev & 4.5 & 16192.466 & od & 4.5 & $0.18 \pm 0.03$ & -1.73 \\
\hline 8693.756 & 29892.677 & od & 3.5 & 18393.327 & ev & 3.5 & $0.61 \pm 0.11$ & -1.26 \\
\hline 8777.180 & 31340.393 & od & 6.5 & 19950.340 & ev & 6.5 & $0.81 \pm 0.12$ & -0.88 \\
\hline 8926.473 & 25681.488 & ev & 1.5 & 14481.930 & od & 2.5 & $0.34 \pm 0.05$ & -1.78 \\
\hline 9014.550 & 27249.669 & ev & 2.5 & 16159.536 & od & 3.5 & $0.099 \pm 0.017$ & -2.14 \\
\hline 9050.375 & 29750.547 & od & 5.5 & 18704.313 & ev & 5.5 & $0.37 \pm 0.06$ & -1.27 \\
\hline 9602.004 & 29892.677 & od & 3.5 & 19481.040 & ev & 4.5 & $0.21 \pm 0.03$ & -1.64 \\
\hline 10433.058 & 27975.619 & od & 4.5 & 18393.327 & ev & 3.5 & $0.102 \pm 0.018$ & -1.78 \\
\hline 10783.013 & 27975.619 & od & 4.5 & 18704.313 & ev & 5.5 & $0.33 \pm 0.05$ & -1.24 \\
\hline
\end{tabular}

(This table is also available in a machine-readable form in the online journal.) 

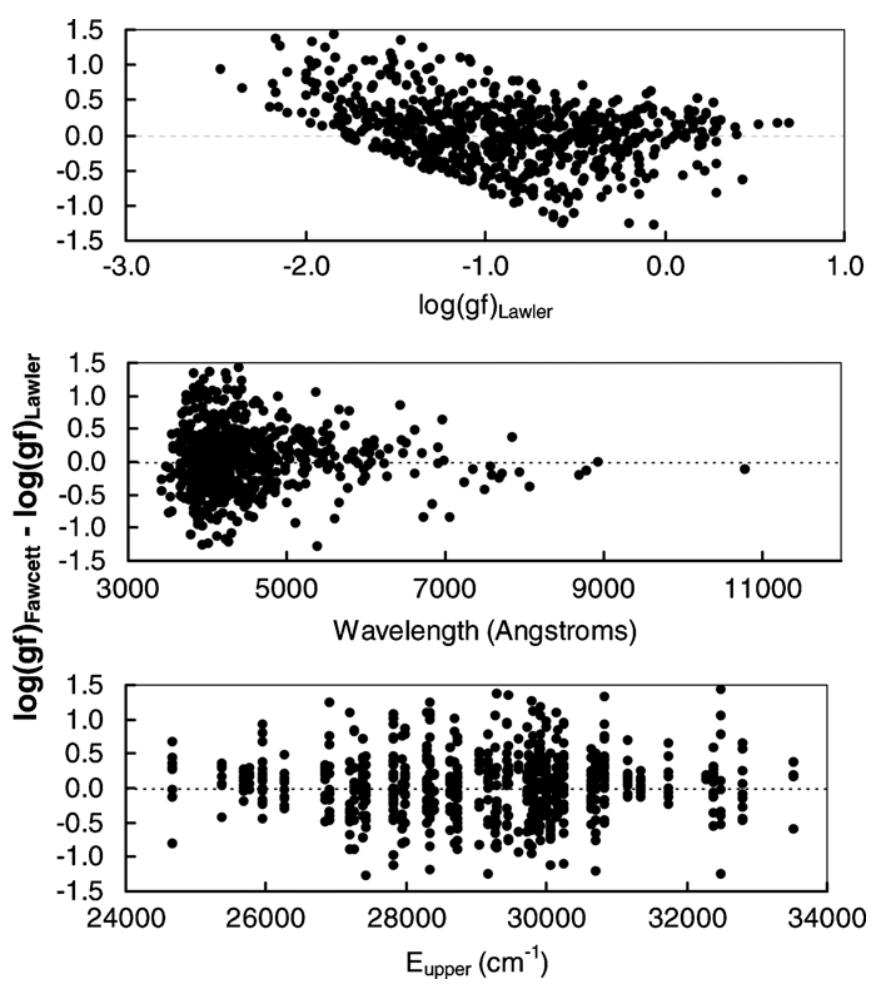

Figure 2. Comparison of theoretical Ce II transition probabilities from Fawcett (1990) to our transition probabilities as function of our transition probability or $\log (g f)$, wavelength, and upper level energy.


Figure 3. Comparison of Ce II transition probabilities recently downloaded from the DREAM database (Biémont \& Quinet 2005) to our transition probabilities as function of our transition probability or $\log (g f)$, wavelength, and upper level energy.

A more substantive question arises about the accuracy of the new experimental results. The agreement between our new experimental results and the various large-scale theoretical and
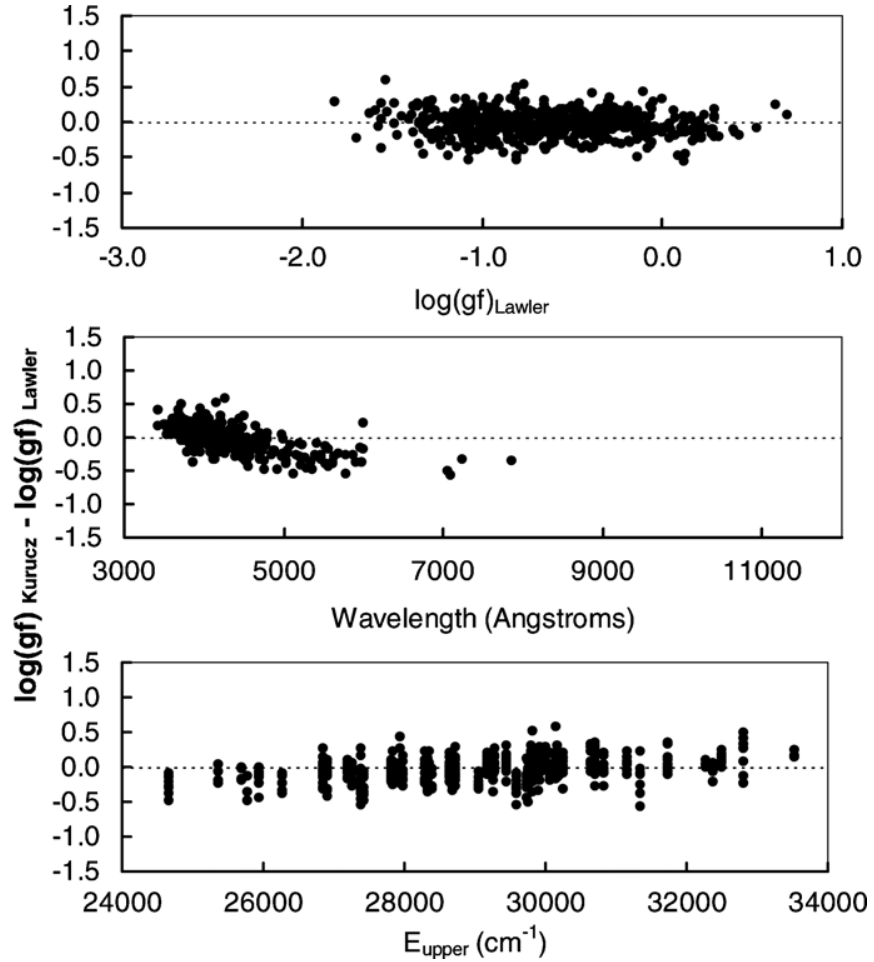

Figure 4. Comparison of semiempirical Ce II transition probabilities recently downloaded from the Kurucz. database (Kurucz 1998) to our transition probabilities as function of our transition probability or $\log (g f)$, wavelength, and upper level energy.

semiempirical data sets is not impressive in any of the above comparisons. One might ask if our experimental error bars are reasonable. This question was recently addressed in a detailed comparison (Lawler et al. 2008a) between Sm II transition probabilities measured as part of this program (Lawler et al. 2006), and independent transition probability measurements from Rehse et al. (2006). Figure 5 shows comparisons of these two experimental data sets for Sm II that were published almost simultaneously. A total of 347 lines in common are included. Lines in common from two levels reported by Rehse et al. and identified as problematic (S. D. Rosner 2007, private communication) are omitted. The two experiments that produced these data sets on Sm II are quite different. A detailed discussion of the relative strengths, weaknesses, and possible systematic errors of both experiments is available (Lawler et al. 2008a). The agreement between these two experimental data sets is much better than the agreement of either with theoretical results.

\section{SOLAR AND STELLAR CERIUM ABUNDANCES}

We used the Ce II transition probabilities to determine new $\mathrm{Ce}$ abundances in the solar photosphere and five very metalpoor $([\mathrm{Fe} / \mathrm{H}]<-2)$, " $r$-process" $n$-capture-rich giant stars. Our procedures followed those used in previous papers of this series; see Den Hartog et al. (2006); Lawler et al. (2006, 2007, 2008b), and references therein.

\subsection{Line Selection}

Cool stars exhibit a rich Ce II spectrum. For example, the solar photospheric line compendium of Moore et al. 1966 lists nearly 200 lines of this species. Therefore identification of transitions suitable for abundance analysis was a straightforward task. We 

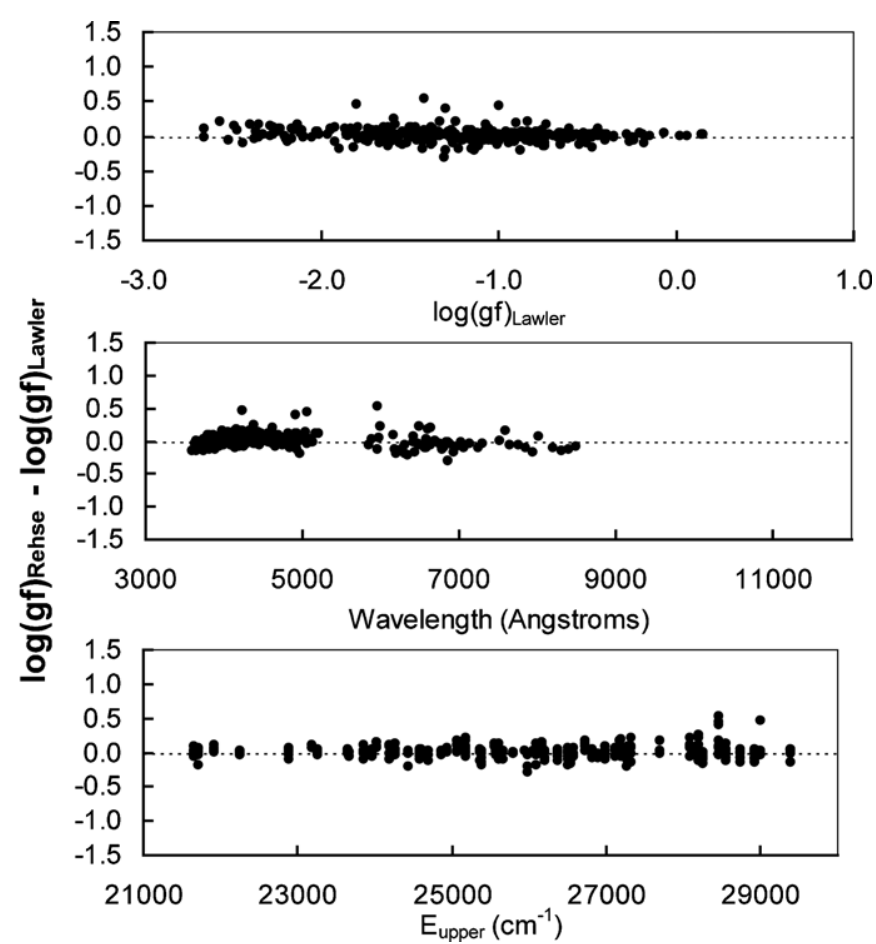

Figure 5. Comparison of experimental Sm II transition probabilities from Rehse et al. (2006) to our transition probabilities as functions of our transition probability or $\log (g f)$, wavelength, and upper level energy. This plot illustrates the level of agreement now achieved between modern, but rather different, experimental techniques.

began by computing relative line strength factors for all 921 Ce II lines of Table 2. As discussed in Lawler et al. (2008b and references therein), the relative absorption strengths of weak-tomoderate lines within a given species are proportional to their transition probabilities modified by their Boltzmann excitation factors. For a line on the linear part of the curve-of-growth this relationship is

$$
\log (\mathrm{RW})=\log (\mathrm{EW} / \lambda)=\text { constant }+\log (\mathrm{g} f)-\theta \chi
$$

where RW is the reduced width, EW is the equivalent width ( $\mathrm{m} \AA$ ), $g f$ is the oscillator strength, $\chi$ is the excitation energy, and $\theta=5040 / \mathrm{T}$ is the inverse temperature.

The relative strengths of lines of different species also depend on their elemental abundances and Saha ionization equilibrium factors. However, Ce, like all REs, has a low first ionization potential: $5.539 \mathrm{eV}$ (Grigoriev \& Melikhov 1997). They all are completely ionized in the stellar photospheres of the Sun and stars of our sample; corrections for their other ionization state populations are negligible. Thus the relative strength factor (STR) of a line can be written as

$$
\mathrm{STR} \equiv \log (\varepsilon \mathrm{g} f)-\theta \chi
$$

where $\varepsilon$ is the elemental abundance. In Figure 6 we plot these relative strength factors as a function of wavelength for $\mathrm{Gd}$ II lines (Den Hartog et al. 2006) and Ce II lines (this paper). For these computations we used solar abundances: $\log \varepsilon(\mathrm{Gd})$ $=+1.11$ (Den Hartog et al. 2006) and $\log \varepsilon(\mathrm{Ce})=+1.55$, which is close to the recommended meteoritic $\mathrm{Ce}$ abundance of $\log \varepsilon(\mathrm{Ce})=+1.58$ (Lodders (2003). We also adopted $\theta=1.0$ for this exercise, roughly the average value of the Sun and the metal-poor giants considered here (since most detectable Gd II and $\mathrm{Ce}$ II transitions in these stars have $\chi<1 \mathrm{eV}$, the exact value of $\theta$ is not important in this exercise).

As in our previous studies, we have drawn horizontal lines in Figure 6 to indicate approximate STR values for "strong" and "barely detectable" lines. These were defined by Lawler et al. (2006) for Sm II, who used the Delbouille et al. (1973) solar photospheric spectrum to estimate the EWs of the weakest lines that could routinely be employed in abundance analyses. The EW limit is $\approx 1.5 \mathrm{~m} \AA$ in the spectral region $\lambda \sim 4500 \AA$, or $\log (\mathrm{RW}) \approx-6.5$. For such Ce II lines, STR $\approx-0.6$. That EW and thus STR limit applies also to Gd II and Ce II lines, as shown in both panels of Figure 6 with horizontal dotted lines. A minimum STR value for relatively strong lines was estimated by Lawler et al. (2006), defining it to be a factor of 20 larger than the line detection limit value, or STR $=-0.6+1.3=+0.7$. We have indicated this "strong-line" limit in Figure 6 with dashed horizontal lines.

Figure 6 displays STR values only in the wavelength range $3000 \AA \leqslant \lambda \leqslant 7000 \AA$ even though our transition probability data extend to nearly $11000 \AA(1.1 \mu \mathrm{m})$. Nearly all Gd II and Ce II lines beyond $7000 \AA$ are undetectably weak in the stars of interest here, having solar relative strengths STR $<$ -1.0. Clearly the "strong" lines of Gd II, Ce II, and nearly all RE ions occur in the blue and near-UV spectral regions $(\lambda<4500 \AA)$, which we emphasize with vertical lines drawn at $4000 \AA$ in the figure. Fortunately, about 70 strong Ce II lines (STR > +0.7) lie longward of $4000 \AA$, and thus are easily accessible to ground-based spectroscopy in a (relatively) un-congested spectral domain. No strong GdII lines are so fortuitously located.

The Ce II STR values were used to trim the original set of 921 transitions (Table 2) to about 620, by discarding those lines weaker than STR $<-0.6$. We then searched through this list to identify potentially useful Ce II lines for abundance analyses. We inspected these lines in the solar photospheric center-of-disk spectrum of Delbouille et al. (1973) and in our spectrum of the $r$-process-rich metal-poor giant star CS 31082-001 $([\mathrm{Fe} / \mathrm{H}]=$ $-2.9,[\mathrm{Eu} / \mathrm{Fe}]=+1.7$, Hill et al. 2002). These spectra, aided by the Moore et al. (1966) solar line identifications and the comprehensive Kurucz (1998) atomic and molecular line lists, showed that the vast majority of candidate $\mathrm{Ce}$ II transitions are too weak and/or too blended to be of use in the Sun and stars of interest here. This process produced a final set of about 60 lines for more detailed study.

\subsection{The Solar Photospheric Cerium Abundance}

We computed synthetic spectra for the CeII lines as in previous papers of this series. Briefly, we started with atomic and molecular line lists in small (4-6 А) wavelength regions, from Kurucz's (1998) line database, supplemented in a few cases by Moore et al.'s (1966) solar identifications. We adopted $g f$-values from laboratory studies for these ionized $n$-capture element species: Y, Hannaford et al. (1982); Zr, Malcheva et al. (2006); La, Lawler et al. (2001a); Ce, this study; Nd, Den Hartog et al. (2003); Sm, Lawler et al. (2006); Eu, Lawler et al. (2001b); Gd, Den Hartog et al. (2006); Tb, Lawler et al. (2001c); Dy, Wickliffe et al. (2000); Ho, Lawler et al. (2004); Er, Lawler et al. (2008b); and Hf, Lawler et al. (2007). The Ce II transitions were treated as single features, since their hyperfine and isotopic substructures are very small.

We adopted the Holweger \& Müller (1974) empirical model photosphere for most of the solar computations. Standard solar abundances were taken from recent reviews (e.g., Grevesse \& 


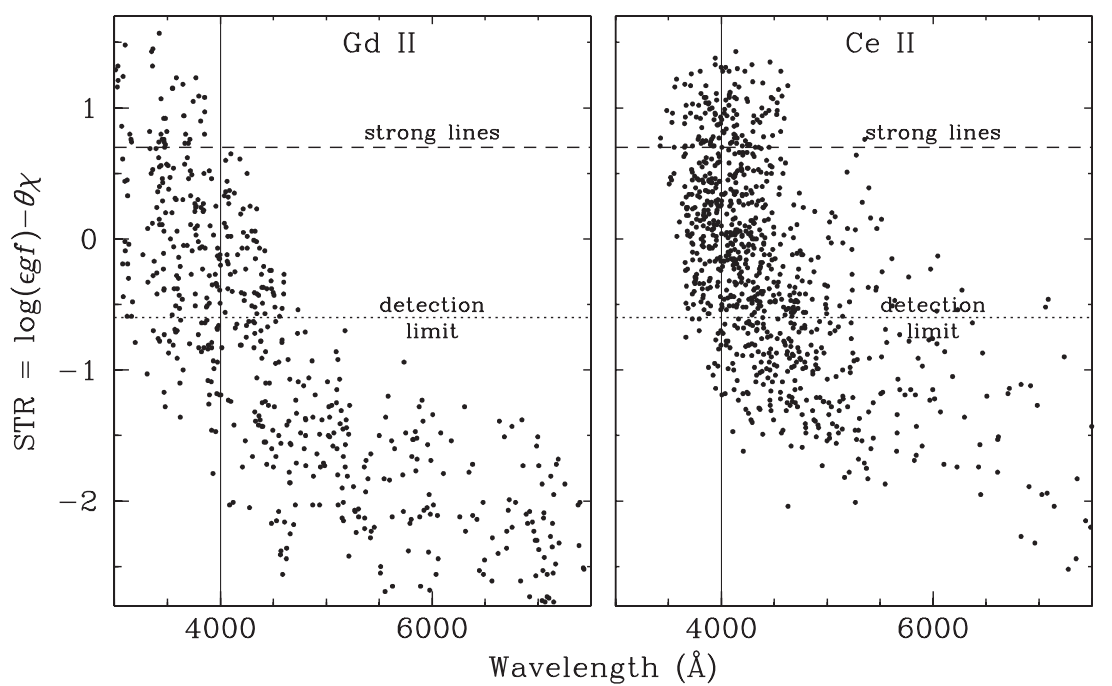

Figure 6. Relative transition strength factors, $\mathrm{STR} \equiv \log (\varepsilon g f)-\theta \chi$, for Gd II (Den Hartog et al. 2006) and Ce II (this study). Definitions of "detection limit" and "strong lines" are given in the text. For display purposes the long-wavelength limit has been set to $7000 \AA$ A. Lines longward of this limit are too weak to be detected in any of the stars of this study. The short-wavelength limit of $3000 \AA$ is defined by the Earth atmospheric transmission cutoff. All Ce II lines of this study are longward of this limit.

Sauval 2002; Lodders 2003; Grevesse et al. 2007), modified for some elements to include recent updates for the $n$-capture elements (given in the papers cited above). We input the solar model, abundance set, and line lists into the current version of the LTE line analysis code MOOG (Sneden 1973) to generate synthetic spectra. These were matched to the observed solar spectrum (Delbouille et al. 1973) after application of empirical Gaussian broadening functions to account for solar macroturbulence and spectrograph instrumental profile.

We computed multiple trial synthetic spectra for each line region. The oscillator strengths for atomic lines other than the $n$-capture species listed above were adjusted to fit the observed solar spectrum. The $\mathrm{C}, \mathrm{N}$, and $\mathrm{O}$ abundances were varied to match observed $\mathrm{CH}, \mathrm{CN}, \mathrm{NH}$, and $\mathrm{OH}$ line strengths. For absorption features without plausible identifications, we arbitrarily attributed them to $\mathrm{Fe}$ I with excitation potentials $\chi=$ $3.5 \mathrm{eV}$ and $g f$-values adjusted to fit the solar spectrum. In cases where line contamination was a significant part of the overall absorption at the Ce II wavelength, the line was discarded for the solar analysis but kept for possible use with the metal-poor giants. After iterating the line list data, final syntheses of 45 Ce II lines were retained for the solar analysis. Individual line abundances are listed in Table 3, along with their excitation energies and $g f$-values. A straight mean abundance is $\log \varepsilon(\mathrm{Ce})$ $=1.61 \pm 0.01(\sigma=0.06)$.

For internal (line-to-line) errors, we estimate line profile fitting uncertainties to be $\pm 0.02 \mathrm{dex}$, contamination by other (non-Ce) features to be \pm 0.02 dex, and $\log (g f)$ uncertainties for the Ce II transitions (see Table 2) are \pm 0.03 . Adding these uncertainties in quadrature yields an estimated total internal uncertainty per transition of $\pm 0.05 \mathrm{dex}$, which is close to the observed $\sigma=0.06$. With solar abundance information from $45 \mathrm{Ce}$ II lines, the mean standard deviation of 0.01 suggests that internal uncertainties are very small contributors to the overall error budget. Scale (external) errors can be due to atomic data uncertainties beyond $g f$ errors, model atmosphere choices, and analysis technique. Since Ce exists almost exclusively as Ce II in these stars, only the Ce II partition function (which enters directly into the Boltzmann equations for our transitions) could contribute to abundance scale uncertainties. Our spectral synthesis code uses Irwin's (1981) partition function polynomial fits to the atomic energy level data available at that time. We re-calculated Ce II partition functions with current energy level information (Martin et al. 2000), but found negligible changes from the Irwin computations in the temperature domain of interest for this study. Finally, in low-metallicity stars, Rayleigh scattering becomes an important continuous opacity source in the blue-UV spectral regions, and radiative transfer source functions can depart somewhat from the Planck function assumed in our calculations. In Sneden et al. (2009) we discuss experiments with more proper accounting for continuum scattering, showing that it produces only small effects for the stars of our sample, and affects most RE elements in a nearly equal fashion, leaving abundance ratios virtually unchanged.

As in Lawler et al. (2007), we repeated some of the abundance computations using the Kurucz (1998) and Grevesse \& Sauval (1999) models, finding average abundance shifts of -0.02 dex compared to those done with the Holweger \& Müller (1974) model. Finally, we emphasize that our analysis is a standard LTE one with a pure Planck source function. Departures from LTE in the ionization equilibrium cannot affect our Ce II analysis because $\mathrm{Ce}$ is already completely ionized. The existence of many low excitation energy levels with this ion increases the likelihood of collisional dominance in their populations. Comments are included in the companion paper (Sneden et al. 2009) on the effect of inclusion of scattering in the continuum source function, but the effect is extremely small for the Sun (and weak for the $r$-process-rich stars). Combining line-to-line scatter uncertainties $( \pm 0.01$ from the standard deviation of the mean, Table 3) with scale uncertainties, we recommend $\log \varepsilon(\mathrm{Ce})_{\text {Sun }}$ $=+1.61 \pm 0.03$. This value is in excellent agreement with the recommended meteoritic abundance of Lodders (2003): $\log$ $\varepsilon(\mathrm{Ce})_{\mathrm{met}}=+1.61 \pm 0.02$.

Palmeri et al. (2000) published the most recent Ce abundance study of the solar photosphere. Analyzing the EWs of 26 lines, they derived $\log \varepsilon(\mathrm{Ce})_{\text {Sun }}=+1.63 \pm 0.04$ from one set of transition probability calculations, and $+1.70 \pm 0.04$ from a different set. We repeated these abundance computations with their EWs, the final recommended $\log (g f)$ values from their web site, and our line analysis code MOOG (Sneden 1973), obtaining 
Table 3

Cerium Abundances from Individual Lines in the Sun and the $r$-Process-Rich Metal-poor Giant Stars

\begin{tabular}{|c|c|c|c|c|c|c|c|c|}
\hline$\lambda(\AA)$ & E. P. $(\mathrm{eV})$ & $\log (\mathrm{gf})$ & $\log \varepsilon$ Sun & $\log \varepsilon \mathrm{BD}+17^{\circ} 3248$ & $\log \varepsilon$ CS 22892 & $\log \varepsilon \operatorname{CS} 31082$ & $\log \varepsilon$ HD 115444 & $\log \varepsilon$ HD 221170 \\
\hline 3534.045 & 0.521 & -0.14 & 1.70 & -0.05 & -0.44 & -0.31 & $\ldots$ & -0.35 \\
\hline 3539.079 & 0.320 & -0.27 & $\ldots$ & -0.10 & -0.47 & $\ldots$ & $\ldots$ & -0.43 \\
\hline 3659.225 & 0.175 & -0.67 & 1.63 & -0.15 & -0.45 & -0.29 & $\ldots$ & -0.41 \\
\hline 3659.970 & 0.175 & -0.73 & 1.63 & $\ldots$ & $\ldots$ & $\ldots$ & $\ldots$ & $\ldots$ \\
\hline 3912.420 & 0.295 & -0.25 & 1.60 & -0.16 & -0.48 & $\ldots$ & $\ldots$ & -0.44 \\
\hline 3942.151 & 0.000 & -0.22 & 1.52 & -0.16 & -0.47 & -0.29 & -1.15 & -0.41 \\
\hline 3942.745 & 0.857 & 0.69 & 1.50 & -0.21 & -0.52 & -0.36 & -1.15 & -0.56 \\
\hline 3953.652 & 0.495 & -0.64 & 1.57 & $\ldots$ & $\ldots$ & $\ldots$ & $\ldots$ & $\ldots$ \\
\hline 3980.890 & 0.708 & -0.21 & 1.70 & -0.13 & $\ldots$ & $\ldots$ & $\ldots$ & $\ldots$ \\
\hline 3993.819 & 0.909 & 0.29 & 1.64 & 0.02 & $\ldots$ & -0.24 & $\ldots$ & $\ldots$ \\
\hline 3999.237 & 0.295 & 0.06 & 1.60 & -0.13 & -0.51 & -0.31 & -1.13 & -0.46 \\
\hline 4042.581 & 0.495 & 0.00 & 1.60 & -0.02 & -0.45 & -0.29 & -1.02 & -0.41 \\
\hline 4053.503 & 0.000 & -0.61 & 1.60 & -0.08 & -0.45 & -0.26 & -1.05 & -0.41 \\
\hline 4068.836 & 0.703 & -0.17 & 1.55 & -0.03 & $\ldots$ & $\ldots$ & $\ldots$ & $\ldots$ \\
\hline 4072.918 & 0.327 & -0.64 & 1.61 & 0.00 & $\ldots$ & -0.22 & $\ldots$ & -0.41 \\
\hline 4073.474 & 0.477 & 0.21 & 1.59 & -0.10 & -0.45 & -0.30 & -1.07 & -0.43 \\
\hline 4075.700 & 0.700 & 0.23 & 1.60 & -0.12 & -0.48 & -0.31 & -1.14 & -0.41 \\
\hline 4083.222 & 0.700 & 0.27 & 1.78 & -0.08 & -0.45 & -0.33 & -1.05 & -0.41 \\
\hline 4117.288 & 0.739 & -0.45 & 1.61 & $\ldots$ & $\ldots$ & -0.31 & $\ldots$ & $\ldots$ \\
\hline 4118.143 & 0.696 & 0.13 & $\ldots$ & -0.08 & -0.43 & -0.27 & -0.97 & -0.38 \\
\hline 4120.827 & 0.320 & -0.37 & 1.70 & -0.08 & -0.30 & -0.22 & -1.00 & -0.37 \\
\hline 4127.364 & 0.683 & 0.31 & 1.60 & -0.08 & -0.47 & -0.31 & -1.05 & -0.44 \\
\hline 4137.645 & 0.516 & 0.40 & 1.75 & -0.10 & -0.45 & -0.28 & -1.05 & -0.38 \\
\hline 4142.397 & 0.696 & 0.22 & $\ldots$ & -0.07 & $\ldots$ & $\ldots$ & $\ldots$ & $\ldots$ \\
\hline 4144.996 & 0.696 & 0.10 & 1.65 & -0.14 & -0.47 & -0.26 & -0.95 & -0.38 \\
\hline 4146.232 & 0.560 & -0.12 & $\ldots$ & -0.10 & -0.47 & -0.26 & -0.90 & $\ldots$ \\
\hline 4222.597 & 0.122 & -0.15 & 1.58 & -0.11 & -0.44 & -0.28 & -1.07 & -0.45 \\
\hline 4337.773 & 0.326 & -0.31 & 1.61 & $\ldots$ & $\ldots$ & $\ldots$ & $\ldots$ & $\ldots$ \\
\hline 4349.768 & 0.529 & -0.73 & $\ldots$ & $\ldots$ & $\ldots$ & $\ldots$ & $\ldots$ & $\ldots$ \\
\hline 4349.789 & 0.700 & -0.32 & 1.60 & -0.18 & -0.50 & -0.29 & $\ldots$ & -0.45 \\
\hline 4364.653 & 0.495 & -0.17 & $\ldots$ & -0.17 & -0.42 & -0.35 & -1.20 & -0.49 \\
\hline 4382.165 & 0.683 & 0.13 & 1.55 & -0.15 & $\ldots$ & -0.28 & $\ldots$ & $\ldots$ \\
\hline 4399.200 & 0.326 & -0.44 & 1.60 & -0.11 & -0.50 & -0.26 & $\ldots$ & -0.38 \\
\hline 4418.780 & 0.863 & 0.27 & 1.65 & -0.14 & $\ldots$ & -0.26 & -1.10 & -0.38 \\
\hline 4449.330 & 0.608 & 0.04 & 1.63 & -0.18 & -0.53 & -0.31 & -1.15 & -0.45 \\
\hline 4486.909 & 0.295 & -0.18 & 1.61 & -0.12 & -0.50 & -0.32 & -1.08 & -0.46 \\
\hline 4523.075 & 0.516 & -0.08 & 1.61 & -0.04 & -0.50 & $\ldots$ & $\ldots$ & $\ldots$ \\
\hline 4560.280 & 0.909 & 0.18 & 1.65 & -0.18 & -0.45 & -0.28 & -1.07 & -0.43 \\
\hline 4560.958 & 0.683 & -0.26 & 1.61 & -0.12 & -0.45 & -0.28 & -1.07 & -0.41 \\
\hline 4562.359 & 0.477 & 0.21 & 1.63 & -0.11 & -0.40 & -0.26 & -1.07 & -0.41 \\
\hline 4572.278 & 0.683 & 0.22 & 1.62 & -0.08 & $\ldots$ & -0.27 & -1.04 & -0.41 \\
\hline 4582.499 & 0.696 & -0.35 & 1.55 & -0.07 & $\ldots$ & -0.30 & -1.07 & -0.38 \\
\hline 4593.926 & 0.696 & 0.07 & 1.65 & -0.13 & -0.45 & -0.27 & -1.02 & -0.41 \\
\hline 4628.161 & 0.516 & 0.14 & 1.57 & -0.09 & -0.37 & -0.26 & -1.05 & -0.41 \\
\hline 4847.914 & 0.956 & -0.75 & 1.52 & $\ldots$ & $\ldots$ & $\ldots$ & $\ldots$ & $\ldots$ \\
\hline 5044.023 & 1.211 & -0.14 & $\ldots$ & $\ldots$ & $\ldots$ & $\ldots$ & $\ldots$ & -0.41 \\
\hline 5187.458 & 1.211 & 0.17 & 1.59 & $\ldots$ & $\ldots$ & -0.31 & $\ldots$ & -0.44 \\
\hline 5274.229 & 1.044 & 0.13 & $\ldots$ & -0.21 & -0.40 & -0.31 & $\ldots$ & -0.46 \\
\hline 5330.556 & 0.869 & -0.40 & 1.70 & $\ldots$ & $\ldots$ & -0.31 & $\ldots$ & -0.48 \\
\hline 5393.392 & 1.102 & -0.06 & 1.55 & $\ldots$ & -0.53 & -0.31 & $\ldots$ & -0.46 \\
\hline 5768.891 & 1.319 & -0.52 & 1.63 & $\ldots$ & $\ldots$ & $\ldots$ & $\ldots$ & $\ldots$ \\
\hline 5975.818 & 1.326 & -0.45 & 1.55 & $\ldots$ & $\ldots$ & $\ldots$ & $\ldots$ & $\ldots$ \\
\hline 6043.373 & 1.205 & -0.48 & 1.64 & $\ldots$ & $\ldots$ & $\ldots$ & $\ldots$ & $\ldots$ \\
\hline Mean & & & 1.61 & -0.11 & -0.46 & -0.29 & -1.06 & -0.42 \\
\hline Unc. & & & 0.01 & 0.01 & 0.01 & 0.01 & 0.01 & 0.01 \\
\hline$\sigma$ & & & 0.06 & 0.05 & 0.05 & 0.03 & 0.07 & 0.04 \\
\hline number of lines & & & 45 & 40 & 32 & 38 & 26 & 37 \\
\hline
\end{tabular}

$\log \varepsilon(\mathrm{Ce})_{\text {Sun }}=+1.67 \pm 0.04(\sigma=0.14)$. Finally, we substituted their $\log (g f)$ values for the ones of the present study for our preferred transitions (Table 3), finding $\log \varepsilon(\mathrm{Ce})_{\text {Sun }}=+1.59 \pm$ $0.02(\sigma=0.10)$. All of these computations are consistent with the overall scale agreement between the Palmeri et al. transition probabilities and those of the present study that was discussed in Section 2.5 .

\subsection{Cerium Abundances in Five r-Process-Rich Low-Metallicity Stars}

The spectra of several very metal-poor, $r$-process-rich giant stars were also analyzed to determine their Ce abundances: $\mathrm{BD}+17^{\circ} 3248([\mathrm{Fe} / \mathrm{H}]=-2.1,[\mathrm{Eu} / \mathrm{Fe}]=+0.9$, Cowan et al. 2002); CS 22892-052 ([Fe/H] $=-3.1,[\mathrm{Eu} / \mathrm{Fe}]=+1.5$, Sneden 


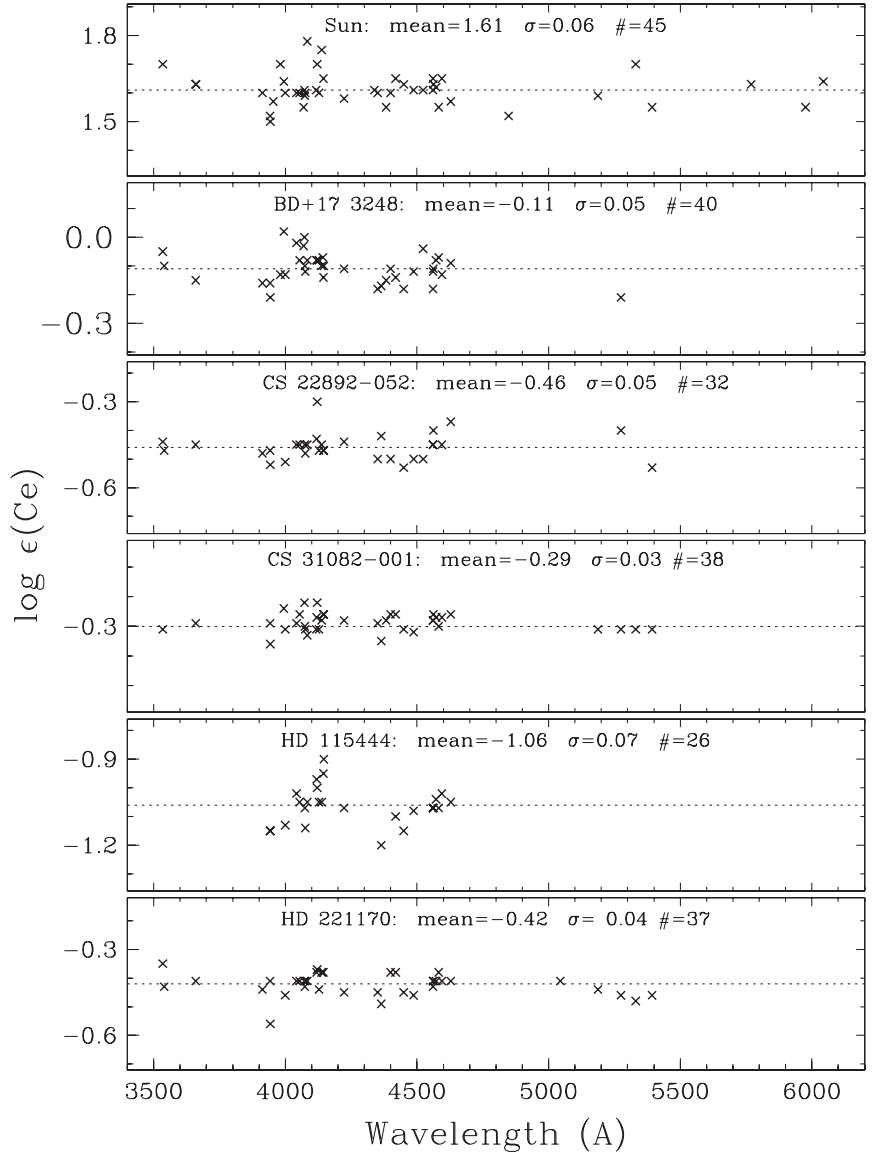

Figure 7. Abundances of individual Ce II lines for all program stars, plotted as a function of wavelength. For each star, a dotted line is drawn at the mean abundance. The mean abundances, sample standard deviation $\sigma$, and the number of lines used in the analyses are given in the legends of each figure panel.

et al. 2003b); CS 31082-001 $([\mathrm{Fe} / \mathrm{H}]=-2.9,[\mathrm{Eu} / \mathrm{Fe}]=+1.7$, Hill et al. 2002); HD $115444([\mathrm{Fe} / \mathrm{H}]=-2.9,[\mathrm{Eu} / \mathrm{Fe}]=$ +0.8 , Westin et al. 2000); and HD $221170([\mathrm{Fe} / \mathrm{H}]=-2.2$, $[\mathrm{Eu} / \mathrm{Fe}]=+0.8$, Ivans et al. 2006). We employed the same analytical methods for these stars as was done for the solar photosphere. The abundances from individual lines are listed in Table 3 and displayed in Figure 7. The average abundances, mean and sample standard deviations, and number of lines are also recorded at the bottom of Table 3 and in Figure 7. The lineto-line scatters are all small, $\sigma=0.03-0.07$, with no obvious trends of abundances as functions of wavelength, $\log (g f)$, or excitation potential. The line-to-line scatters for individual stars approximately anti-correlate with their Ce II line strengths. We derived the smallest scatter $(\sigma=0.03)$ for CS 31082-001, which has the strongest lines, and the largest $(\sigma=0.07)$ for HD 115444 , with mostly very weak lines.

\section{DISCUSSION AND CONCLUSIONS}

We compare the newly derived $\mathrm{Ce}$ abundances among the five $r$-rich stars in Figure 8. The differences between the observed Ce abundances for each star-BD+17 3248 , CS 22892-052, CS 31082-001, HD 115444, and HD 221170 - and the solar system $r$-process only value (Arlandini et al. 1999) are illustrated in the figure. In all cases, the abundances have been normalized with respect to the Eu abundances. (These abundances have also been newly redetermined for those five stars and are reported in the companion paper; Sneden et al. 2009.) The corresponding

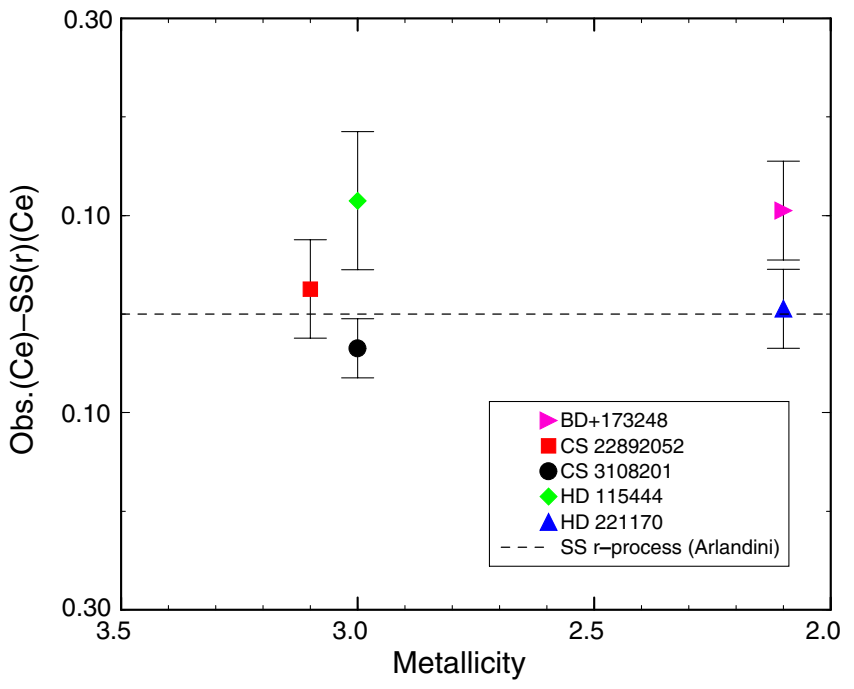

Figure 8. Comparison of the newly determined Ce abundances in five $r$-processrich stars to the solar system $r$-process only value (Arlandini et al. 1999). For each star the abundances have been normalized at Eu. The dashed line indicates a perfect agreement between the stellar and solar system $r$-only values for Ce. The error bars are the sigma values listed for each star in Table 3.

(A color version of this figure is available in the online journal.)

error bars are those given for $\log \varepsilon(\mathrm{Ce})$ in Table 3. As is clear from the figure, the Ce abundances in all five $r$-rich stars are in general agreement with a solar system $r$-only value and there is little scatter among the stars - with the largest deviations from the solar $r$-process value (indicated by a dashed line) of approximately 0.1 dex. (A value of zero would thus indicate a perfect agreement with that prediction of the solar system $r$-only values.) The two stars deviating the most from the solar system $r$-process values are HD 115444 and BD $17^{\circ} 3248$. The increased precision of our new $\mathrm{Ce}$ abundance determinations suggests that the error limits are typically less than $0.1 \mathrm{dex}$. Thus, it is possible that there might be some small $s$-process contamination to the Ce abundances in those two stars. (Ce is a predominantly $s$ process element, $81 \%$, in solar system material.) If, however, this slight $s$-process contribution is due to Galactic $s$-process nucleosynthesis, we would expect to see an increasing trend in Ce abundance as a function of metallicity. Previous studies (see Burris et al. 2000 and Simmerer et al. 2004) found evidence of Galactic $s$-process nucleosynthesis at metallicities less than -2.0 .

We see in Figure 8 that (somewhat surprisingly) there is no metallicity effect on the agreement between the Ce stellar and solar system $r$-process only ratios. There is an almost perfect agreement with the $r$-process only value for Ce in CS 22892 052 at $[\mathrm{Fe} / \mathrm{H}]=-3.1$ and in $\mathrm{HD} 221170$ at $[\mathrm{Fe} / \mathrm{H}]=-2.1$. Since $\mathrm{Ce}$ is produced predominantly in the $s$-process in solar system material (see e.g., Simmerer et al. 2004), it might be expected that there would be some rise in the $\mathrm{Ce}$ abundance at metallicities near -2 . While $\mathrm{BD}+17^{\circ} 3248$ does show a rise at that point, there is no clear overall trend, at least for this sample of only five $r$-rich stars. Thus, there may be something specific to these two stars that would indicate Ce abundances slightly larger than, but still roughly in agreement with, the predicted $r$-process only ratios. Studies of possible small $s$-process contributions to other $s$-process-dominant RE elements, such as $\mathrm{Ba}$, in these five stars studied here are included in Sneden et al. (2009).

This consistency between stellar and solar-system $r$-processonly values for RE elements is not surprising and has been reported previously for each of these stars: $\mathrm{BD}+17^{\circ} 3248$ (Cowan 
et al. 2002), CS 22892-052 (Sneden et al. 2004), CS 31082001 (Hill et al. 2002), HD 115444 (Westin et al. 2000) and HD 221170 (Ivans et al. 2006). However, in the past there has been considerably more star-to-star scatter in individual elements, including Ce (see Figure 10 in Lawler et al. 2008b). This scatter has now been dramatically reduced for Ce with much smaller error bars than previously possible, as a result of the new atomic physics data presented in this paper. Further discussion and analyses of other RE elements, based upon new atomic data, can be found in the companion paper Sneden et al. (2009).

This work has been supported by the National Science Foundations through grants AST-0506324 to JEL \& EDH, AST0607708 to CS, and AST-0707447 to J.J.C. J.E.L. is a guest observer at the NSO on Kitt Peak, AZ.

\section{APPENDIX}

This work on Ce II, as indicated in the Introduction, completes a multiyear effort to improve laboratory spectroscopic data for RE ions and to apply these data in abundance studies. This appendix is a summary of lab data from the project including transition probabilities, isotopic shifts, hfs constants, and complete isotopic and hfs line component patterns. References are included to published data tables as well as some new MR tables. A few of our early papers did not include MR tables of transition probability data, and we have since had numerous requests for such MR tables. In other cases we published only isotope shifts and/or hfs constants without realizing that many astronomers would prefer complete isotopic and/or hyperfine line component patterns. We are here providing such tables.

We also wish to correct a typographical error in a hfs energy formula which appeared in several of our papers (Lawler et al. 2001b, 2001d, 2004). The typographical error did not affect any of our tables of hfs constants or tables of complete line component patterns (CLCPs). We are using the Casimir formula as presented in the elementary text by Woodgate (1980). This correct formula is

$$
\Delta E=\frac{A K}{2}+B \frac{3 K(K+1)-4 I(I+1) J(J+1)}{8 I(2 I-1) J(2 J-1)},
$$

where $\Delta E$ is the shift in wave numbers of an hfs sublevel $(F, J)$ from the center of gravity of the fine structure level $(J)$,

$$
K=F(F+1)-J(J+1)-I(I+1),
$$

$F$ is the total atomic angular momentum, $J$ is the total electronic angular momentum, and $I$ is the nuclear spin. The more advanced text by Cowan (1981) has an excellent discussion of hfs, but uses a slightly different definition of the hyperfine B. Relative intensities of hyperfine components are expressed in terms of Wigner 6-j symbols by Cowan (1981). Some readers may find it more convenient to use the simple Russell-Saunders line strength formulae given in the classic text by Condon \& Shortley (1935). The use of the Russell-Saunders formulae only requires substitution of $F$ for $J, J$ for $L$, and $I$ for $S$.

Each RE element has its own nuclear configuration, and each first ion of these elements has a unique electronic energy structure. However, some general properties that connect many of the $Z=57-71$ ions are worth noting. Among the seven stable odd- $Z$ elements of this group, five of them have only one naturally-occurring isotope. $\mathrm{Lu}(Z=71)$ has stable isotopes ${ }^{175} \mathrm{Lu}$ and ${ }^{176} \mathrm{Lu}$, but ${ }^{175} \mathrm{Lu}$ comprises $97.4 \%$ of the solar system $\mathrm{Lu}$ abundance. This essentially leaves only Eu as the odd-Z RE element with multiple abundant isotopes: ${ }^{151} \mathrm{Eu}(47.8 \%$ of the $\mathrm{Eu}$ elemental abundance in the solar system), and ${ }^{153} \mathrm{Eu}(52.2 \%)$. Additionally, the $\mathrm{Eu}$ isotopic fraction appears to be relatively insensitive to the $n$-capture synthesis method, being nearly $50 /$ 50 in both the $r$-process and $s$-process (e.g., Sneden et al. 2002; Aoki et al. 2003). Therefore once the hyperfine patterns for odd$Z$ rare-earth ionized transitions are known, the spectra can be synthesized with confidence.

In contrast, all but one of the even- $Z$ elements in this element domain have at least five naturally-occurring non-negligible isotopes ( $>1 \%$ fraction in the solar system). The single exception is $\mathrm{Ce}$, with just ${ }^{140} \mathrm{Ce}(88.5 \%)$ and ${ }^{142} \mathrm{Ce}(11.1 \%)$. The odd-A isotopes of these elements have non-zero nuclear magnetic moments, and thus their ionized-species transitions have usually small hfs. However, the isotopic wavelength shifts are usually undetectably small. Magain (1995) and Lambert \& Allende Prieto (2002) studied the isotopic mix of $\mathrm{Ba}$, a near RE element, from the BaII $4554 \AA$ line in one very metal-poor star, but their results had large error bars because the isotopic wavelength shifts are only $\sim 0.05 \AA$. Roederer et al. (2008) derived $\mathrm{Sm}$ isotopic abundance fractions from several lines of $\mathrm{Sm}$ II in metal-poor stars, but the isotopic splits were always $<\sim 0.08 \AA$. In both of these examples the isotopic wavelength spreads are comparable to the stellar thermal and microturbulent broadening of the lines. No other RE even- $Z$ element appears to exhibit significant isotopic broadening in spectroscopically accessible transitions (see attempts to analyze Nd II by Roederer et al.), so for most purposes their lines can be treated as single features.

Table 4 is a summary of laboratory data measured or compiled as part of this program on RE ions with locations of MR tables of $\log (g f)$ values and of CLCPs. The general discussion above is supplemented with more detailed discussions of the spectra of each RE ion below. As the quality of stellar spectra from large and very large ( $>6 \mathrm{~m}$ ) telescopes continues to improve, there will be increased opportunities for elemental abundance studies and isotopic fraction measurements on many more stars. Complete and very accurate tables of solar system isotopic fractions and nuclear moments are available, but are not included here (Böhlke et al. 2005; Stone 2005).

Lanthanum is an on odd- $Z$ element with essentially one stable isotope, ${ }^{139} \mathrm{La}$, with hfs from a nuclear spin $I=7 / 2$ which is significant in stellar abundance work. The very low isotopic fraction of ${ }^{138} \mathrm{La}$ of $0.09 \%$ in solar system material is negligible. When our work on La II was published (Lawler et al. 2001a) we were not aware of the ApJ option to include MR tables. Table 5 is an MR version of the La II transition probability table from the 2001 paper. A set of hfs constants for ${ }^{139}$ La was measured and/or compiled by Lawler et al. (2001a) and updated by Ivans et al. (2006) with an MR table of CLCPs.

Cerium is covered in this paper. It is an even- $Z$ element with only nuclear spin $I=0$ isotopes. Two isotopes have solar system fractions above $10 \%$, but the isotopes shifts are generally small. There are also two rare $I=0$ isotopes with solar system fractions $<1 \%$. Lines of Ce II are sufficiently narrow in our FTS data that they may be treated as single component lines in stellar abundance work.

Praseodymium is discussed in the companion paper (Sneden et al. 2009). It is an odd- $Z$ element with a single stable isotope, ${ }^{141} \mathrm{Pr}$, with hfs from a nuclear spin $I=5 / 2$ which is significant in stellar abundance work. The companion paper has a recommended set of $\log (g f)$ values and an MR table of CLCPs.

Neodymium is an even- $Z$ element with five $I=0$ isotopes and two $I=7 / 2$ isotopes, all with fractions of $5 \%$ or more 
Table 4

Master Table of Laboratory Data Measured or Compiled as Part of This Program on RE ions

\begin{tabular}{|c|c|c|c|c|}
\hline Spectrum & $Z$ & $\log (g f)$ & $\begin{array}{l}\text { Isotope Data Shifts and/or hfs } \\
\text { Constants }\end{array}$ & Location of MR Tables \\
\hline La II & 57 & Lawler et al. 2001a & Lawler et al. 2001a; Ivans et al. 2006 & $\begin{array}{c}\log (g f): \text { MR Table } 5 \text { below } \\
\text { CLCP: MR Table } 4 \text { in Ivans et al. } 2006\end{array}$ \\
\hline Ce II & 58 & This paper & $\ldots$ & $\log (g f):$ MR Table 2 above \\
\hline Pr II & 59 & $\begin{array}{l}\text { Companion paper: } \\
\text { Sneden et al. } 2009\end{array}$ & $\begin{array}{l}\text { Companion paper: } \\
\text { Sneden et al. } 2009\end{array}$ & $\begin{array}{c}\log (g f) \text { : Companion paper } \\
\text { CLCP: MR Table } 11 \text { in Sneden et al. } 2009\end{array}$ \\
\hline $\mathrm{Nd}$ II & 60 & Den Hartog et al. 2003 & Roederer et al. 2008 & $\begin{array}{c}\log (g f): \text { MR Table } 3 \text { in Den Hartog et al. } 2003 \\
\text { CLCP: MR Table } 5 \text { in Roederer et al. } 2008\end{array}$ \\
\hline Pm II & 61 & no stable isotopes & no stable isotopes & no stable isotopes \\
\hline Sm II & 62 & Lawler et al. 2006 & Roederer et al. 2008 & $\begin{array}{l}\log (g f): \text { MR Table } 2 \text { of Lawler et al. } 2006 \\
\text { CLCP: MR Table } 4 \text { of Roederer et al. } 2008\end{array}$ \\
\hline Eu II & 63 & Lawler et al. 2001b & Lawler et al. 2001b; Ivans et al. 2006 & $\begin{array}{c}\log (g f) \text { : MR Table } 6 \text { below } \\
\text { CLCP: MR Table } 6 \text { of Ivans et al. } 2006\end{array}$ \\
\hline Gd II & 64 & Den Hartog et al. 2006 & $\cdots$ & $\log (g f):$ MR Table 3 of Den Hartog et al. 2006 \\
\hline $\mathrm{Tb}$ II & 65 & Lawler et al. 2001c & Lawler et al. 2001d & $\begin{array}{c}\log (g f): \text { MR Table } 1 \text { of Lawler et al. 2001c } \\
\text { CLCP: MR Table } 7 \text { below }\end{array}$ \\
\hline Dy II & 66 & Wickliffe et al. 2000 & $\ldots$ & $\log (g f):$ MR Table 8 below \\
\hline Ho II & 67 & Lawler et al. 2004 & Lawler et al. 2004 & $\begin{array}{l}\log (g f): \text { MR Table } 9 \text { below } \\
\text { CLCP: MR Table } 10 \text { below }\end{array}$ \\
\hline Er II & 68 & Lawler et al. $2008 b$ & $\cdots$ & $\log (g f):$ MR Table 3 of Lawler et al. 2008b \\
\hline Tm II & 69 & Wickliffe \& Lawler 1997 & $\cdots$ & $\log (g f):$ MR Table 11 below \\
\hline Yb II & 70 & $\begin{array}{l}\text { Companion paper: } \\
\text { Sneden et al. } 2009\end{array}$ & $\begin{array}{l}\text { Companion paper: } \\
\text { Sneden et al. } 2009\end{array}$ & $\begin{array}{c}\log (g f): \text { Companion paper } \\
\text { CLCP: MR Table } 12 \text { in Sneden et al. } 2009\end{array}$ \\
\hline Lu II & 71 & $\begin{array}{l}\text { Den Hartog et al. 1998; } \\
\text { Quinet et al. 1999; Fedchak et al. } 2000\end{array}$ & Sneden et al. $2003 \mathrm{~b}$ & $\begin{array}{l}\log (g f): \text { MR Table } 13 \text { below } \\
\text { CLCP: MR Table } 14 \text { below }\end{array}$ \\
\hline
\end{tabular}

Note. Machine-readable (MR) tables of $\log (g f)$ values and complete line component patterns (CLCPs) are now available for these data either in the original publication or in this Appendix.

Table 5

Experimental Atomic Transition Probabilities for La II Arranged by Wavenumber from Lawler et al. (2001a)

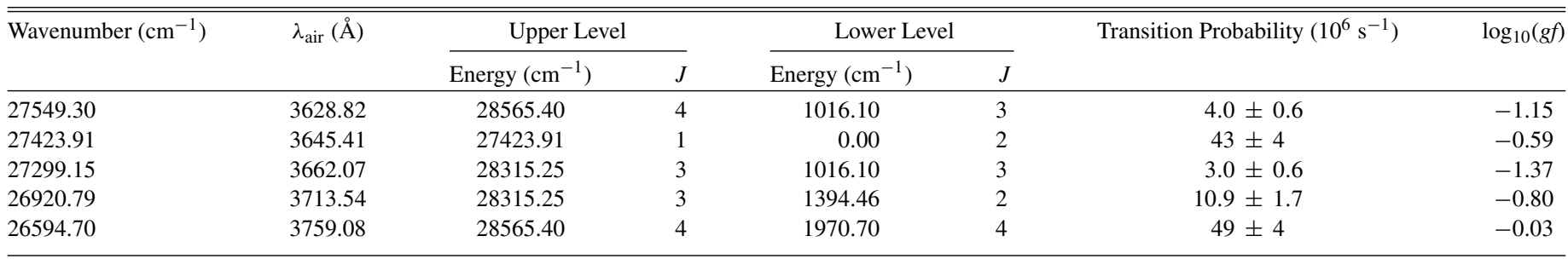

(This table is available in its entirety in a machine-readable form in the online journal. A portion is shown here for guidance regarding its form and content.)

in solar system material. Some lines of Nd II have structure in our FTS data as noted in Table 3 of Den Hartog et al. (2006) but these lines tended to be weak in stellar spectra. Most lines of Nd II are sufficiently narrow in our FTS data to be treated as single component lines in stellar abundance work. Roederer et al. (2008) compiled Nd II isotope shifts and hfs constants, and published an MR tables of CLCPs. Isotopic fraction measurements on $\mathrm{Nd}$ in stellar abundance work have been attempted, but with only limited success because the isotope shifts are so small. Since there is no resolved structure in Nd II lines in stellar spectra, tiny center-of-gravity shifts of $\mathrm{Nd}$ II lines must be measured in stellar spectra to determine isotopic fractions.

Prometheum has no stable isotopes. Although there have been tentative observations of Pm II lines in stellar spectra (e.g., Cowley et al. 2004), none have been confirmed.

Samarium is an even- $Z$ element with five $I=0$ isotopes and two $I=7 / 2$ isotopes, all with fractions of $3 \%$ or more in solar system material. Most lines of Sm II are sufficiently narrow in our FTS data to be treated as single component lines in stellar abundance work. Roederer et al. (2008) compiled Sm II isotope shifts and hfs constants, and published an MR table of CLCPs. Isotopic fraction measurements on $\mathrm{Sm}$ in stellar abundance work are somewhat easier than on $\mathrm{Nd}$, and such measurements have been performed (Roederer et al. 2008). Small center-of-gravity shifts of Sm II lines were measured in stellar spectra to determine isotopic fractions.

Europium is an odd- $Z$ element with two $I=5 / 2$ isotopes, both with large, $47.8 \%$ and $52.2 \%$, isotopic fractions in solar system material. When our work on Eu II was published (Lawler et al. 2001b) we did not include MR tables. Table 6 is an MR version of the Eu II transition probability table from the 2001 paper. A set of hfs constants for ${ }^{151} \mathrm{Eu}$ and ${ }^{153} \mathrm{Eu}$ was compiled by Lawler et al. (2001b). The energy levels and center-of gravity wavelengths of Eu II transition were improved by Ivans et al. 
Table 6

Experimental Atomic Transition Probabilities for Eu II Arranged by Wavenumber from Lawler et al. (2001b)

\begin{tabular}{|c|c|c|c|c|c|c|c|}
\hline \multirow[t]{2}{*}{ Wavenumber $\left(\mathrm{cm}^{-1}\right)$} & \multirow[t]{2}{*}{$\lambda_{\text {air }}(\AA)$} & \multicolumn{2}{|c|}{ Upper Level } & \multicolumn{2}{|c|}{ Lower Level } & \multirow[t]{2}{*}{ Transition Probability $\left(10^{6} \mathrm{~s}^{-1}\right)$} & \multirow[t]{2}{*}{$\log _{10}(g f)$} \\
\hline & & Energy $\left(\mathrm{cm}^{-1}\right)$ & $J$ & Energy $\left(\mathrm{cm}^{-1}\right)$ & $J$ & & \\
\hline 27104.07 & 3688.43 & 27104.07 & 3 & 0.00 & 4 & $15.0 \pm 1.1$ & -0.67 \\
\hline 26838.50 & 3724.93 & 26838.50 & 4 & 0.00 & 4 & $43.3 \pm 2.6$ & -0.09 \\
\hline 26172.83 & 3819.67 & 26172.83 & 5 & 0.00 & 4 & $135 \pm 7$ & 0.51 \\
\hline 25587.14 & 3907.11 & 27256.35 & 2 & 1669.21 & 3 & $129 \pm 6$ & 0.17 \\
\hline 25434.86 & 3930.50 & 27104.07 & 3 & 1669.21 & 3 & $114 \pm 6$ & 0.27 \\
\hline
\end{tabular}

(This table is available in its entirety in a machine-readable form in the online journal. A portion is shown here for guidance regarding its form and content.)

Table 7


Standard Index of Air (Edlén 1953)

\begin{tabular}{|c|c|c|c|c|c|c|}
\hline Wavenumber $\left(\mathrm{cm}^{-1}\right)$ & $\lambda_{\text {air }}(\AA)$ & $\mathrm{F}_{\text {upp }}$ & $\mathrm{F}_{\text {low }}$ & Component Position $\left(\mathrm{cm}^{-1}\right)$ & Component Position $(\AA)$ & Strength \\
\hline 32563.20 & 3070.059 & 8.5 & 8.5 & -0.14668 & 0.013830 & 0.29338 \\
\hline 32563.20 & 3070.059 & 8.5 & 7.5 & 0.24576 & -0.023171 & 0.00662 \\
\hline 32563.20 & 3070.059 & 7.5 & 8.5 & -0.42038 & 0.039636 & 0.00662 \\
\hline 32563.20 & 3070.059 & 7.5 & 7.5 & -0.02794 & 0.002634 & 0.25128 \\
\hline 32563.20 & 3070.059 & 7.5 & 6.5 & 0.31834 & -0.030014 & 0.00877 \\
\hline
\end{tabular}

Notes.

Center-of-gravity wavenumbers and air wavelengths, $\lambda_{\text {air }}$, are given with component positions relative to those values. Strengths are normalized to sum to 1 .

(This table is available in its entirety in a machine-readable form in the online journal. A portion is shown here for guidance regarding its form and content.)

Table 8

Experimental Atomic Transition Probabilities for Dy I (Levels Have Integral $J$ ) and Dy II (Levels Have Half Integral $J$ ) from Wickliffe et al. (2000)

\begin{tabular}{|c|c|c|c|c|c|c|c|c|c|c|c|c|c|}
\hline \multirow{2}{*}{$\begin{array}{l}\text { Wavenumber } \\
\left(\mathrm{cm}^{-1}\right)\end{array}$} & \multirow[t]{2}{*}{$\lambda_{\text {air }}(\AA)$} & \multicolumn{3}{|c|}{ Upper Level } & \multicolumn{3}{|c|}{ Lower Level } & \multicolumn{3}{|c|}{ Univ. of Wisconsin } & \multicolumn{3}{|c|}{ NIST } \\
\hline & & $\begin{array}{l}\text { Energy } \\
\left(\mathrm{cm}^{-1}\right)\end{array}$ & Parity & $J$ & $\begin{array}{l}\text { Energy } \\
\left(\mathrm{cm}^{-1}\right)\end{array}$ & Parity & $J$ & $\begin{array}{c}\text { Transition } \\
\text { Probability }\left(10^{6} \mathrm{~s}^{-1}\right)\end{array}$ & Uncert.(\%) & $\log _{10}(g f)$ & $\begin{array}{c}\text { Transition } \\
\text { Probability }\end{array}$ & Uncert. (\%) & $\log _{10}(g f)$ \\
\hline 34921.87 & 2862.69 & 34921.87 & od & 7.0 & 0.00 & ev & 8.0 & 7.9 & 6 & -0.84 & $\ldots$ & $\ldots$ & $\ldots$ \\
\hline 33311.52 & 3001.09 & 33311.52 & od & 7.0 & 0.00 & ev & 8.0 & 1.45 & 6 & -1.53 & $\ldots$ & $\ldots$ & $\ldots$ \\
\hline 33165.77 & 3014.28 & 33165.77 & od & 8.0 & 0.00 & ev & 8.0 & 1.18 & 7 & -1.56 & $\ldots$ & .. & $\ldots$ \\
\hline 33035.57 & 3026.16 & 33035.57 & od & 9.5 & 0.00 & ev & 8.5 & 3.82 & 6 & -0.98 & $\ldots$ & . & $\ldots$ \\
\hline 32490.90 & 3076.89 & 33319.21 & od & 6.5 & 828.31 & ev & 7.5 & 1.35 & 10 & -1.57 & $\ldots$ & $\ldots$ & $\ldots$ \\
\hline
\end{tabular}

(This table is available in its entirety in a machine-readable form in the online journal. A portion is shown here for guidance regarding its form and content.)

Table 9

Experimental Atomic Transition Probabilities for Ho II Arranged by Wavenumber from Lawler et al. (2004)

\begin{tabular}{|c|c|c|c|c|c|c|c|c|}
\hline \multirow[t]{2}{*}{ Wavenumber $\left(\mathrm{cm}^{-1}\right)$} & \multirow[t]{2}{*}{$\lambda_{\text {air }}(\AA)$} & \multicolumn{2}{|c|}{ Upper Level } & \multicolumn{2}{|c|}{ Lower Level } & \multirow[t]{2}{*}{ Transition Probability $\left(10^{6} \mathrm{~s}^{-1}\right)$} & \multicolumn{2}{|c|}{$\log _{10}(g f)$} \\
\hline & & Energy $\left(\mathrm{cm}^{-1}\right)$ & $J$ & Energy $\left(\mathrm{cm}^{-1}\right)$ & $J$ & & This Expt. & Other \\
\hline 30918.86 & 3233.34 & 31556.61 & 7 & 637.75 & 7 & $4.1 \pm 0.8$ & -1.02 & \\
\hline 29899.58 & 3343.57 & 29899.58 & 7 & 0.00 & 8 & $14.1 \pm 1.1$ & -0.45 & $-0.54^{\mathrm{a}}$ \\
\hline 29450.17 & 3394.59 & 35067.62 & 6 & 5617.46 & 7 & $16.1 \pm 1.1$ & -0.44 & \\
\hline 29412.51 & 3398.94 & 29412.51 & 8 & 0.00 & 8 & $87 \pm 5$ & 0.41 & $0.27^{\mathrm{b}}$ \\
\hline 29325.35 & 3409.04 & 29325.35 & 7 & 0.00 & 8 & $3.05 \pm 0.21$ & -1.10 & \\
\hline
\end{tabular}

Notes.

a VALD database as described in Kupka et al. (1999), value determined using the method of Magazzù \& Cowley (1986).

b VALD database as described in Kupka et al. (1999), value originally from Gorshkov \& Komarovskii (1979).

(This table is available in its entirety in a machine-readable form in the online journal. A portion is shown here for guidance regarding its form and content.)

(2006) and CLCPs were provided in MR Table 6 of that paper. Astrophysical data on halo stars from modern large telescopes have now reached the level of quality that isotopic abundances of Eu can be determined with some accuracy and precision (e.g., Sneden et al. 2002; Aoki et al. 2003). Evidence to date supports a uniform isotopic mix from all $r$-process events. The lines of Eu II connected to ground and lowest metastable levels are ideal for such studies. The unpaired s-electron of these two levels yields both large isotope shifts from the finite nuclear size (field shifts) and wide hfs. Isotope shifts and hfs yield resolvable structure in Eu II lines in stellar spectra, which is much easier to measure than the small wavelength shifts of Sm II or Nd II lines due to varying isotopic fractions.

Gadolinium is an even- $Z$ element with five $I=0$ isotopes and two $I=3 / 2$ isotopes. The two lightest $I=0$ isotopes have rather low fractions $<2.5 \%$ in solar system material. Most lines of Gd II are sufficiently narrow in our FTS data to be treated as single component lines in stellar abundance work. The widths of line profiles in our highest resolution FTS data vary, and in a few cases the profiles have partially resolved structure. Although it is 
Table 10

Hyperfine Structure Line Component Patterns for Ho II Computed Using hfs Constants and Improved Energy Levels from Lawler et al. (2004) and the Standard Index of Air (Edlén 1953)

\begin{tabular}{lcrrrr}
\hline \hline Wavenumber $\left(\mathrm{cm}^{-1}\right)$ & $\lambda_{\text {air }}(\AA)$ & $\mathrm{F}_{\text {upp }}$ & $\mathrm{F}_{\text {low }}$ & Component Position $\left(\mathrm{cm}^{-1}\right)$ & Component Position $(\AA)$ \\
\hline 30918.859 & 3233.3387 & 10.5 & 10.5 & 0.81266 & -0.084984 \\
30918.859 & 3233.3387 & 10.5 & 9.5 & 0.80394 & -0.084073 \\
30918.859 & 3233.3387 & 9.5 & 10.5 & 0.46722 & -0.048860 \\
30918.859 & 3233.3387 & 9.5 & 9.5 & 0.45850 & -0.047949 \\
30918.859 & 3233.3387 & 9.5 & 8.5 & 0.45885 & -0.047985 \\
\hline
\end{tabular}

Note. Center-of-gravity wavenumbers and air wavelengths, $\lambda_{\text {air }}$, are given with component positions relative to those values. Strengths are normalized to sum to 1 . (This table is available in its entirety in a machine-readable form in the online journal. A portion is shown here for guidance regarding its form and content.)

Table 11

Experimental Atomic Transition Probabilities for Tm II from Wickliffe \& Lawler (1997)

\begin{tabular}{|c|c|c|c|c|c|c|c|c|c|c|}
\hline \multirow[t]{2}{*}{ Wavenumber $\left(\mathrm{cm}^{-1}\right)$} & \multirow[t]{2}{*}{$\lambda_{\text {air }}(\AA)$} & \multicolumn{3}{|c|}{ Upper Level } & \multicolumn{3}{|c|}{ Lower Level } & \multirow{2}{*}{$\begin{array}{c}\text { Transition } \\
\text { Probability }\left(10^{6} \mathrm{~s}^{-1}\right)\end{array}$} & \multirow[t]{2}{*}{ Uncert.(\%) } & \multirow[t]{2}{*}{$\log _{10}(g f)$} \\
\hline & & Energy $\left(\mathrm{cm}^{-1}\right)$ & Parity & $\bar{J}$ & Energy $\left(\mathrm{cm}^{-1}\right)$ & Parity & $\bar{J}$ & & & \\
\hline 34913.84 & 2863.35 & 34913.84 & ev & 3 & 0.00 & od & 4 & 1.05 & 8 & -2.04 \\
\hline 34842.39 & 2869.22 & 47299.68 & od & 7 & 12457.29 & $\mathrm{eV}$ & 6 & 225 & 6 & 0.62 \\
\hline 34634.42 & 2886.45 & 34871.37 & ev & 4 & 236.95 & od & 3 & 1.16 & 11 & -1.88 \\
\hline 34580.75 & 2890.93 & 34580.75 & ev & 5 & 0.00 & od & 4 & 5.23 & 5 & -1.14 \\
\hline 34307.52 & 2913.96 & 34307.52 & ev & 4 & 0.00 & od & 4 & 1.11 & 5 & -1.89 \\
\hline
\end{tabular}

(This table is available in its entirety in a machine-readable form in the online journal. A portion is shown here for guidance regarding its form and content.)

not possible today, it may at some point in the future be possible to observe the isotopic mixture of $\mathrm{Gd}$ in a metal-poor halo star.

Terbium is an on odd- $Z$ element with one stable isotope, ${ }^{159} \mathrm{~Tb}$, with hfs from a nuclear spin $I=3 / 2$ which is significant in stellar abundance work. Lawler et al. (2001d) published a large set of hfs constants. CLCPs for lines of Tb II are in MR Table 7.

Dysprosium is an even- $Z$ element with five $I=0$ isotopes and two $I=5 / 2$ isotopes. The three lightest $I=0$ isotopes have rather low fractions $<2.5 \%$ in solar system material. Although some lines have detectable structure, most lines of Dy II are sufficiently narrow in our FTS data to be treated as single component lines in stellar abundance work. Wickliffe et al. (2000) measured a large set of Dy I and Dy II transition probabilities in this effort on RE species. This work on Dy was done jointly with NIST and comparisons of independent branching fraction measurements were performed. Both the Univ. of Wisconsin (UW) and NIST transition probabilities are based on the same radiative lifetimes measured at UW using timeresolved LIF. Wickliffe et al. published a merged table of Dy I and Dy II transition probabilities with both UW and NIST measurements. This table has been reconstructed here as an MR Table 8.

Holmium is an odd- $Z$ element with a single stable isotope, ${ }^{165} \mathrm{Ho}$, and hfs from a nuclear spin $I=7 / 2$ which is significant in stellar abundance work. The high line density of Ho I and Ho II in combination with wide hfs has inhibited analysis of Ho II. Lawler et al. (2004) focused their efforts on the lowlying levels which are connected by strong "resonance-like" transitions. They reported new transition probability measurements based on radiative lifetimes from LIF in combination with branching fractions from FTS data, a small set of improved energy levels from FTS data for better center-of-gravity wavelengths, and hfs constants from FTS data for the levels of interest. Their table of transition probabilities is reproduced as an MR Table 9 below, and MR Table 10 includes CLCPs for the strong "resonance-like" lines of Ho II.

Erbium is an even- $Z$ element with five $I=0$ isotopes and one $I=7 / 2$ isotope. The two lightest $I=0$ isotopes have rather
Table 12

Improved Lu II Energy Levels $\left( \pm 0.008 \mathrm{~cm}^{-1}\right)$ and NIST (Martin et al. 1978) Energy Levels for Comparison

\begin{tabular}{lrr}
\hline \hline $\begin{array}{l}\text { Energy }\left(\mathrm{cm}^{-1}\right) \\
\text { This Expt. }\end{array}$ & NIST & $J$ \\
\hline 0.000 & 0.00 & 0 \\
11796.108 & 11796.24 & 1 \\
12435.229 & 12435.32 & 2 \\
14198.978 & 14199.08 & 3 \\
17332.428 & 17332.58 & 2 \\
27264.269 & 27264.40 & 0 \\
28503.149 & 28503.16 & 1 \\
32453.115 & 32453.26 & 2 \\
41224.821 & 41224.96 & 2 \\
44918.529 & 44918.68 & 3 \\
48536.758 & 48536.83 & 4 \\
45458.326 & 45458.56 & 2 \\
\hline
\end{tabular}

low fractions $<2 \%$ in solar system material. Although some lines have detectable structure, most lines of Er II are sufficiently narrow in our FTS data to be treated as single component lines in stellar abundance work. Some of the IR lines studied by Lawler et al. (2008b) have detectable isotopic structures, but these lines are so weak with such high excitation potentials that there is no hope of astrophysical detections in the foreseeable future.

Thulium is an odd- $Z$ element with one stable isotope, ${ }^{169} \mathrm{Tm}$. This isotope has a nuclear spin of $I=1 / 2$. Although some lines have detectable structure, most lines of Tm II are sufficiently narrow in our FTS data to be treated as single component lines in stellar abundance work. Wickliffe and Lawler (1997) measured a large set of Tm I and Tm II transition probabilities in this effort on RE species. Their data on Tm II are included below as an MR Table 11.

Ytterbium is discussed in the companion paper (Sneden et al. 2009). It is an even- $Z$ element with five $I=0$ isotopes, one $I=1 / 2$ isotope, and one $I=5 / 2$ isotope. The lightest two $I=0$ isotopes have rather low fractions, $<3.1 \%$, in solar system material. Only two $\mathrm{Yb}$ II resonance lines from the same multiplet are useful in abundance studies and thus an MR table of 
Table 13

Experimental Atomic Transition Probabilities for Lu II from Odd-Parity Upper Levels Organized by Increasing Wavelength in Air

\begin{tabular}{|c|c|c|c|c|c|c|}
\hline \multirow[t]{2}{*}{$\lambda_{\text {air }}(\AA)$} & \multicolumn{2}{|c|}{ Upper Level } & \multicolumn{2}{|c|}{ Lower Level } & \multirow[t]{2}{*}{ Transition Probability $\left(10^{6} \mathrm{~s}^{-1}\right)$} & \multirow[t]{2}{*}{$\log _{10}(g f)$} \\
\hline & Energy $\left(\mathrm{cm}^{-1}\right)$ & $J$ & Energy $\left(\mathrm{cm}^{-1}\right)$ & $J$ & & \\
\hline 2571.23 & 53079.33 & 3 & 14199.08 & 3 & $39.8 \pm 2.7$ & -0.56 \\
\hline 2796.63 & 53079.33 & 3 & 17332.58 & 2 & $181 \pm 9$ & 0.17 \\
\hline 2894.84 & 48733.19 & 3 & 14199.08 & 3 & $186 \pm 12$ & 0.21 \\
\hline 2911.3915 & 48536.758 & 4 & 14198.978 & 3 & $245 \pm 16$ & 0.45 \\
\hline 3254.3173 & 44918.529 & 3 & 14198.978 & 3 & $64 \pm 4$ & -0.15 \\
\hline 3397.0669 & 41224.821 & 2 & 11796.108 & 1 & $75 \pm 5$ & -0.19 \\
\hline 3472.4832 & 41224.821 & 2 & 12435.229 & 2 & $71 \pm 5$ & -0.19 \\
\hline 3507.3810 & 28503.149 & 1 & 0.000 & 0 & $12.5 \pm 1.1$ & -1.16 \\
\hline 3623.9806 & 44918.529 & 3 & 17332.428 & 2 & $11.9 \pm 1.4$ & -0.79 \\
\hline 4184.2533 & 41224.821 & 2 & 17332.428 & 2 & $21.0 \pm 2.0$ & -0.56 \\
\hline 4839.6201 & 32453.115 & 2 & 11796.108 & 1 & $0.45 \pm 0.05$ & -2.10 \\
\hline 5476.6884 & 32453.115 & 2 & 14198.978 & 3 & $21.2 \pm 1.1$ & -0.32 \\
\hline 5983.8429 & 28503.149 & 1 & 11796.108 & 1 & $4.3 \pm 0.4$ & -1.16 \\
\hline 6221.8596 & 28503.149 & 1 & 12435.229 & 2 & $9.9 \pm 0.9$ & -0.76 \\
\hline 6463.1065 & 27264.269 & 0 & 11796.108 & 1 & $15.4 \pm 0.8$ & -1.01 \\
\hline 8459.16 & 41224.96 & 2 & 29406.70 & 2 & $2.5 \pm 0.5$ & -0.87 \\
\hline
\end{tabular}

Notes.

Updated energy levels from Table 12 are used for transitions if available both for the upper and lower levels, otherwise NIST (Martin et al. 1978) energy levels are used. Wavelengths are computed using the standard index of air (Edlén 1953).

(This table is also available in a machine-readable form in the online journal.)

Table 14

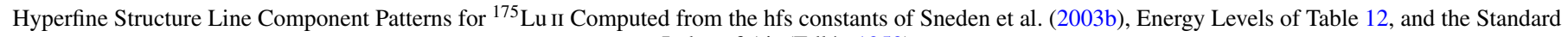
Index of Air (Edlén 1953)

\begin{tabular}{lccccr}
\hline \hline Wavenumber $\left(\mathrm{cm}^{-1}\right)$ & $\lambda_{\text {air }}(\AA)$ & $\mathrm{F}_{\text {upp }}$ & $\mathrm{F}_{\text {low }}$ & ${\text { Component Position }\left(\mathrm{cm}^{-1}\right)}^{\text {Component Position }(\AA)}$ & Strength \\
\hline 34337.780 & 2911.3915 & 7.5 & 6.5 & -0.53033 & 0.044968 \\
34337.780 & 2911.3915 & 6.5 & 6.5 & -0.62922 & 0.053353 \\
34337.780 & 2911.3915 & 6.5 & 5.5 & -0.22156 & 0.0222 \\
34337.780 & 2911.3915 & 5.5 & 6.5 & -0.67036 & 0.16827 \\
34337.780 & 2911.3915 & 5.5 & 5.5 & -0.26270 & 0.00160 \\
\end{tabular}

Notes.

Center-of-gravity wavenumbers and air wavelengths, $\lambda_{\text {air }}$, are given with component positions relative to those values. Strengths are normalized to sum to 1 . Table 14 is available in its entirety via the link to the machine-readable version above.

(This table is available in its entirety in a machine-readable form in the online journal. A portion is shown here for guidance regarding its form and content.)

transition probabilities is unnecessary. The unpaired s-electron of the ground level yields large isotope shifts and hfs. Isotopic and hfs are not negligible in stellar abundance work on Yb II. The companion paper has recommended $\log (g f)$ values and an MR table of CLCPs.

Lutetium is an odd- $Z$ element with one dominant stable isotope, ${ }^{175} \mathrm{Lu}$, with hfs from a nuclear spin $I=7 / 2$ which is significant in stellar abundance work. The low isotopic fraction of ${ }^{176} \mathrm{Lu}$ of $2.59 \%$ in solar system material is negligible given the generally low abundance of Lu and quality of stellar spectra available today. During a search for Lu in metal-poor stars, it became apparent that the NIST energy levels (Martin et al. 1978) are not as accurate as the NIST energy levels for many other species. Energy levels from older measurements using photographic techniques on species with wide hfs often have this problem. Table 12 presents new energy level measurements for some of the low-lying levels of Lu II of both parities. Lutetium was studied early in this project on RE ions, shortly after Bord et al. (1998) found a clean Lu II line in the solar spectrum at $6221 \AA$ A. Den Hartog et al. (1998) measured the upper level life- time of this line using LIF and the branching fractions of lines from this level using FTS data to determine accurate transition probabilities. Shortly thereafter, additional branching fractions from FTS data were reported by Quinet et al. (1999) and additional radiative lifetimes from time-resolved LIF were reported by Fedchak et al. (2000). In this early phase of the RE project we collaborated more closely with atomic theorists and performed numerous comparisons of experimental and theoretical transition probabilities. With only two valence electrons, Lu II is a particularly simple RE spectrum with minimal configuration mixing. However, both core polarization and relativistic effects are important in Lu II like other RE spectra. At this time we are of the opinion that careful branching fraction measurements from FTS data in combination with radiative lifetimes from LIF generally yield better RE transition probabilities than can be computed using ab initio quantum methods. We therefore recommend the Lu II transition probabilities of Table 13 that were determined by combining branching fractions from FTS data with radiative lifetimes from our time-resolved LIF experiment. Sneden et al. (2003b) measured a set of hfs constants from FTS data for 
levels of ${ }^{175} \mathrm{Lu}$. For the user's convenience MR Table 14 includes CLCPs for lines of ${ }^{175} \mathrm{Lu}$ II. The reader should note that the components from the rare $(2.59 \%)$ isotope ${ }^{176} \mathrm{Lu}$ are not included in Table 14. New energy levels from Table 12 are included in Tables 13 and 14 if available for both the upper and lower levels of the transition, otherwise energy levels from Martin et al. (1978) are used. Air wavelengths are computed from those energy levels using the standard index of air (Edlén 1953).

\section{REFERENCES}

Adams, D. L., \& Whaling, W. 1981, J. Opt. Soc. Am., 71, 1036

Aoki, W., Honda, S., Beers, T. C., \& Sneden, C. 2003, ApJ, 586, 506

Arlandini, C., Käppeler, F., Wisshak, K., Gallino, R., Lugaro, M., Busso, M., \& Straniero, O. 1999, ApJ, 525, 886

Biémont, E., \& Quinet, P. 2005, J. Electron Spectrosc. Relat. Phenom., 144, 23

Böhlke, J. K., de Laeter, J. R., De Bièvre, P., Hidaka, H., Peiser, H. S., Rosman,

K. J. R., \& Taylor, P. D. P. 2005, J. Phys. Chem. Ref. Data, 34, 57

Bord, D. J., Cowley, C. R., \& Mirijanian, D. 1998, Sol. Phys., 178, 221

Brault, J. W. 1976, J. Opt. Soc. Am., 66, 1081

Burris, D. L., Pilachowski, C. A., Armandroff, T. E., Sneden, C., Cowan, J. J., \& Roe, H. 2000, ApJ, 544, 302

Condon, E. U., \& Shortley, G. H. 1935, The Theory of Atomic Spectra (Cambridge: Cambridge Univ. Press), 238

Cowley, C. R., Bidelman, W. P., Hubrig, S., Mathys, G., \& Bord, D. J. 2004, A\&A, 419, 1087

Cowan, J. J., Sneden, C., Truran, J. W., \& Burris, D. L. 1996, ApJ, 460, L115

Cowan, J. J., et al. 2002, ApJ, 572, 861

Cowan, R. D. 1981, The Theory of Atomic Structure and Spectra (Berkeley, CA: Univ. of California Press), 508

Danzmann, K., \& Kock, M. 1982, J. Opt. Soc. Am., 72, 1556

Delbouille, L., Roland, G., \& Neven, L. 1973, Photometric Atlas of the Solar Spectrum from lambda 3000 to lambda 10000 (Liège, Inst. d'Ap.: Univ. de Liège)

Den Hartog, E. A., Curry, J. J., Wickliffe, M. E., \& Lawler, J. E. 1998, Sol. Phys., 178, 239

Den Hartog, E. A., \& Lawler, J. E. 2008, J. Phys. B: At. Mol. Opt. Phys., 41, 045701

Den Hartog, E. A., Lawler, J. E., Sneden, C., \& Cowan, J. J. 2003, ApJS, 148, 543

Den Hartog, E. A., Lawler, J. E., Sneden, C., \& Cowan, J. J. 2006, ApJS, 167, 292

Edlén, B. 1953, J. Opt. Soc. Am., 43, 339

Fawcett, B. C. 1990, At. Nucl. Data Tables, 46, 217

Fedchak, J. A., Den Hartog, E. A., Lawler, J. E., Palmeri, P., Quinet, P., \& Biémont, E. 2000, ApJ, 542, 1109

Grevesse, N., Asplund, M., \& Sauval, A. J. 2007, Space Sci. Rev., 130, 105

Grevesse, N., \& Sauval, A. J. 1999, A\&A, 347, 348

Grevesse, N., \& Sauval, A. J. 2002, Adv. Space. Res., 30, 3

Grigoriev, I. S., \& Melikhov, E. Z. 1997, Handbook of Physical Quantities (Boca Raton, FL: CRC Press), 516

Hannaford, P., Lowe, R. M., Grevesse, N., Biémont, E., \& Whaling, W. 1982, ApJ, 261, 736

Hashiguchi, S., \& Hasikuni, M. 1985, J. Phys. Soc. Japan, 54, 1290

Hill, V., et al. 2002, A\&A, 387, 560

Holweger, H., \& Müller, E. A. 1974, Sol. Phys., 39, 19

Irwin, A. W. 1981, ApJS, 45, 621

Ivans, I. I., Simmerer, J., Sneden, C., Lawler, J. E., Cowan, J. J., Gallino, R., \& Bisterzo, S. 2006, ApJ, 645, 613

Kurucz, R. L. 1998, in IAU Symp. 189, Fundamental Stellar Properties: The Interaction between Observation and Theory, ed. T. R. Bedding, A. J. Booth, \& J. Davis (Dordrecht: Kluwer), 217

Lambert, D. L., \& Allende Prieto, C. 2002, MNRAS, 335, 325
Langhans, G., Schade, W., \& Helbig, V. 1995, Z. Phys. D, 34, 155

Lawler, J. E., Bonvallet, G., \& Sneden, C. 2001a, ApJ, 556, 452

Lawler, J. E., Den Hartog, E. A., Labby, Z. E., Sneden, C., Cowan, J. J., \& Ivans, I. I. 2007, ApJS, 169, 120

Lawler, J. E., Den Hartog, E. A., Sneden, C., \& Cowan, J. J. 2006, ApJS, 162 227

Lawler, J. E., Den Hartog, E. A., Sneden, C., \& Cowan, J. J. 2008a, Can. J. Phys., 86, 1033

Lawler, J. E., Sneden, C., \& Cowan, J. J. 2004, ApJ, 604, 850

Lawler, J. E., Sneden, C., Cowan, J. J., Wyart, J.-F., Ivans, I. I., Sobeck, J. S., Stockett, M. H., \& Den Hartog, E. A. 2008b, ApJS, 178, 71

Lawler, J. E., Wickliffe, M. E., Cowley, C. R., \& Sneden, C. 2001c, ApJS, 137, 341

Lawler, J. E., Wickliffe, M. E., Den Hartog, E. A., \& Sneden, C. 2001b, ApJ, 563,1075

Lawler, J. E., Wyart, J.-F., \& Blaise, J. 2001d, ApJS, 137, 351

Li, Z. S., Lundberg, H., Wahlgren, G. M., \& Sikström, C. M. 2000, Phys. Rev. A, 62,032505

Lodders, K. 2003, ApJ, 591, 1220

Malcheva, G., Blagoev, K., Mayo, R., Ortiz, M., Xu, H. L., Svanberg, S., Quinet, P., \& Biémont, E. 2006, MNRAS, 367, 754

Magain, P. 1995, A\&A, 297, 686

Martin, W. C., Sugar, J., \& Musgrove, A. 2000, NIST Atomic Spectra Database, (http://physics.nist.gov/PhysRefData/ASD/index.html)

Martin, W. C., Zalubas, R., \& Hagan, L. 1978, Atomic Energy Levels The Rare Earth Elements, NSRDS NBS 60 (Washington, DC: US Govt Printing Office), 70

Moore, C. E., Minnaert, M. G. J., \& Houtgast, J. 1966, The Solar Spectrum $2934 \AA$ Å $8770 \AA ̊$ Å, NBS Monograph 61 (Washington, DC: US Govt Printing Office)

Palmeri, P., Quinet, P., Wyart, J.-F., \& Biémont, E. 2000, Phys. Scr., 61, 323

Quinet, P., Palmeri, P., Biémont, E., McCurdy, M. M., Rieger, G., Pinnington, E. H., Wickliffe, M. E., \& Lawler, J. E. 1999, MNRAS, 307, 934

Rehse, S. J., Li, R., Scholl, T. J., Sharikova, A., Chatelain, R., Holt, R. A., \& Rosner, S. D. 2006, Can. J. Phys., 84, 723

Roederer, I. U., Lawler, J. E., Sneden, C., Cowan, J. J., Sobeck, J. S., \& Pilachowski, C. A. 2008, ApJ, 675, 723

Simmerer, J., Sneden, C., Cowan, J. J., Collier, J., Woolf, V. M., \& Lawler, J. E. 2004, ApJ, 617, 1091

Smith, V. V., Cunha, K., \& Lambert, D. L. 1995, AJ, 110, 2827

Sneden, C. 1973, ApJ, 184, 839

Sneden, C., Basri, G., Boesgarrd, A. M., Brown, J. A., Carney, B. W., Kraft, R. P., Smith, V., \& Suntzeff, N. B. 1995, PASP, 107, 997

Sneden, C., Cowan, J. J., \& Lawler, J. E. 2003a, Nucl. Phys. A, 718, 29c

Sneden, C., Cowan, J. J., Lawler, J. E., Burles, S., Beers, T. C., \& Fuller, G. M. 2002, ApJ, 566, L25

Sneden, C., Lawler, J. E., Cowan, J. J., Ivans, I. I., \& Den Hartog, E. A. 2009 ApJS, 182, 80

Sneden, C., McWilliam, A., Preston, G. W., Cowan, J. J., Burris, D. L., \& Armosky, B. J. 1996, ApJ, 467, 819

Sneden, C., et al. 2003b, ApJ, 591, 936

Stone, N. J. 2005, At. Data Nucl. Data Tables, 90, 75

Westin, J., Sneden, C., Gustafsson, B., \& Cowan, J. J. 2000, ApJ, 530, 783

Whaling, W., Carle, M. T., \& Pitt, M. L. 1993, J. Quant. Spectrosc. Radiat. Transfer, 50, 7

Wickliffe, M. E., \& Lawler, J. E. 1997, J. Opt. Soc. Am. B, 14, 737

Wickliffe, M. E., Lawler, J. E., \& Nave, G. 2000, J. Quant. Spectrosc. Radiat. Transfer, 66, 363

Woolf, V. M., Tomkin, J., \& Lambert, D. L. 1995, ApJ, 453, 660

Woodgate, G. K. 1980, Elementary Atomic Structure,2nd edn. (Oxford: Clarendon), 184

Xu, H. L., Persson, A., \& Svanberg, S. 2003, Eur. Phys. J. D, 23, 233

Zhang, Z. G., Svanberg, S., Jiang, Z., Palmeri, P., Quinet, P., \& Biémont, E. 2001, Phys. Scr., 63, 122 